\title{
MILNOR'S PROBLEM ON THE GROWTH OF GROUPS AND ITS CONSEQUENCES
}

\author{
ROSTISLAV GRIGORCHUK \\ Dedicated to John Milnor on the occasion of his 80th birthday.
}

\begin{abstract}
We present a survey of results related to Milnor's problem on group growth. We discuss the cases of polynomial growth and exponential but not uniformly exponential growth; the main part of the article is devoted to the intermediate (between polynomial and exponential) growth case. A number of related topics (growth of manifolds, amenability, asymptotic behavior of random walks) are considered, and a number of open problems are suggested.
\end{abstract}

\section{INTRODUCTION}

The notion of the growth of a finitely generated group was introduced by A.S. Schwarz (also spelled Schvarts and Švarc)) [Š55] and independently by Milnor [Mil68b, Mil68a]. Particular studies of group growth and their use in various situations have appeared in the works of Krause [Kra53], Adelson-Velskii and Shreider [AVŠ57], Dixmier [Dix60], Dye [Dye59, Dye63], Arnold and Krylov [AK63], Kirillov [Kir67], Avez [Ave70], Guivarc'h [Gui70, Gui71, Gui73], Hartley, Margulis, Tempelman and other researchers. The note of Schwarz did not attract a lot of attention in the mathematical community, and was essentially unknown to mathematicians both in the USSR and the West (the same happened with papers of Adelson-Velskii, Dixmier and of some other mathematicians). By contrast, the note of Milnor [Mil68a], and especially the problem raised by him in [Mil68b], initiated a lot of activity and opened new directions in group theory and areas of its applications.

The motivation for Schwarz and Milnor's studies on the growth of groups were of geometric character. For instance, it was observed by Schwarz that the rate of volume growth of the universal cover $\tilde{M}$ of a compact Riemannian manifold $M$ coincides with the rate of growth of the fundamental group $\pi_{1}(M)$ [Š55]. At the same time Milnor and Wolf demonstrated that growth type of the fundamental group gives some important information about the curvature of the manifold. A relation between the growth of a group and its amenability was discovered by Adel'son-Vel'skii and Shreider (spelled also as Šreider) [AVŠ57]; specifically, subexponential growth implies the amenability of the group, i.e., the existence of an invariant mean).

The problem posed by Milnor focuses on two main questions:

(1) Are there groups of intermediate growth between polynomial and exponential?

2000 Mathematics Subject Classification. 20F50,20F55,20E08.

Key words and phrases. growth of group, growth function, growth series, Milnor problem, polynomial growth, intermediate growth, exponential growth, uniformly exponential growth, gap conjecture, amenable group, self-similar group, branch group, group generated by finite automaton.

The author is supported by NSF grant DMS-1207699, ERS grant GA 257110 "RaWG", and by the Simons Foundation. 
(2) What are the groups with polynomial growth?

Moreover, Milnor formulated a remarkable conjecture about the coincidence of the class of groups with polynomial growth and the class of groups containing a nilpotent subgroup of finite index (i.e. with the class of virtually nilpotent groups) which was later proved by Gromov [Gro81a].

The first part of Milnor's question was formulated originally by him in the following form: "Is it true that the growth function of every finitely generated group is necessarily equivalent to a polynomial or to the function $2^{n}$ ?" This was answered in the negative by the author in 1983 [Gri83, Gri84b]. Despite the negative character of the answer, the existence of groups of intermediate growth made group theory and the areas of its applications much richer. Eventually it led to the appearance of new directions in group theory: self-similar groups [GNS00, Nek05], branch groups [Gri00a, Gri00b, BGŠ03], and iterated monodromy groups [BGN03, Nek05]. Moreover, completely new methods, used in the study of groups of intermediate growth, stimulated intensive studies of groups generated by finite automata - a direction on the border between computer science and algebra initiated by V.M. Glushkov in the beginning of the 1960s [Glu61, KAP85, GNS00, BGK ${ }^{+} 08$ ]. Groups of intermediate growth were used and continue to be used in many different situations: in the study of amenable groups [Gri84b, Gri98], in topology [FT95], in theory of random walks on groups [Ers10, Kai05, Ers04a], in theory of operator algebras [Gri05, GN07, Gri], in dynamical systems [BN08, BP06], in percolation [MP01b], in the study of cellular automata [MM93], in the theory of Riemannian surfaces [Gri89b], etc.

As was already mentioned, the study of the growth of groups was introduced by Schwarz and Milnor. However, some preliminary cases were considered by H.U. Krause in his Ph.D thesis, defended in Zurich in 1953 [Kra53], and geometric growth was considered around 1953 by V.A. Efremovich [Efr53]. Later, the question of group growth was considered in certain situations by Adelson-Velskii and Shreider, Dixmier, Dye, Arnold and Krylov, Guivarc'h, Kirillov, Margulis, Tempelman, and other researchers; however, as has been already mentioned, the systematic study of group growth only started with Milnor's work in 1965.

The first period of studies concerning group growth (in the 1960s and 1970s) was dedicated to the study of growth of nilpotent and solvable groups. During a short period of time, Milnor, Wolf, Hartley, Guivarc'h and Bass discovered that nilpotent groups have polynomial growth of integer degree, given by a number (6.2) expressed in terms of the lower central series. The converse fact, namely that polynomial growth implies the virtual nilpotence of the group was proven by M. Gromov in his remarkable paper [Gro81a], which stimulated a lot of activity in different areas of mathematics. Gromov's proof uses the idea of the limit of a sequence of metric spaces, as well as Montgomery and Zippin's solution of Hilbert's 5th problem [MZ74]. Van den Dries and Wilkie [vdDW84] used methods of nonstandard analysis to explore Gromov's idea in order to slightly improve his result (in particular, to outline a broader approach to the notion of a cone of a group), while B. Kleiner, using the ideas of Colding-Minicozzi [CM97] and applying the techniques of harmonic functions, gave a proof of Gromov's theorem which doesn't rely on the techniques in the Montgomery-Zipin solution to Hilbert's 5th problem. This approach was explored by Y. Shalom and T. Tao to get effective and quantitative results about the polynomial growth case.

The author's paper [Gri89a], followed by the ICM Kyoto paper [Gri91], raised a very interesting question which we discuss in detail in this article (section 10) and call the Gap Conjecture. The conjecture states that if the growth degree of a finitely generated group 
is strictly smaller than $e^{\sqrt{n}}$ then it is polynomial and the group is virtually nilpotent. If proven, this conjecture would give a far reaching generalization of Gromov's polynomial growth theorem. There are several results, some presented here, supporting the conjecture.

It is interesting that the $p$-adic analogue of Hilbert's 5th Problem, solved by M. Lazard [Laz65], was used by the author [Gri89a, Gri91] to obtain results related to the Gap Conjecture; these are discussed in section 10. See also the work of A. Lubotzky, A. Mann and D. Segal [LM91, LMS93] for a description of the groups with polynomial subgroup growth.

The notion of growth can be defined for many algebraic and combinatorial objects, in particular for semigroups, associative and Lie algebras, graphs and discrete metric spaces as discussed, for instance, in [dlHGCS99]. There is much more freedom in the asymptotic behavior of growth in each of these classes of algebraic objects, and there is no hope to obtain a general result characterizing polynomial growth similar to Gromov's theorem. But in [Gri88], the author was able to extend the theorem of Gromov to the case of cancellative semigroups (using the notion of nilpotent semigroup introduced by A.I. Malcev [Mal53]).

Let us say a few words about the exponential growth case. Perhaps typically, a finitely generated group has exponential growth. The fact that any solvable group which is not virtually nilpotent has exponential growth was established by Milnor and Wolf in 1968 [Mil68c, Wol68]. A direct consequence of the Tits Alternative [Tit72] is that a finitely generated linear group has exponential growth if it is not virtually nilpotent.

The exponential growth of a group immediately follows from the existence of a free subgroup on two generators, or even a free sub-semigroup on two generators. There are many classes of groups (i.e. solvable non-virtually nilpotent groups, non-elementary Gromov hyperbolic groups, linear groups without free subgroup on two generators, etc.) which are known to contain such objects. But many infinite torsion groups also have exponential growth, as was first proved by S.I. Adian in the case of free Burnside groups of large odd exponent [Adi79].

The second period of studies of group growth begins in the 1980s and splits into three directions: the study of analytic properties of growth series $\Gamma(z)=\sum_{n=0}^{\infty} \gamma(n) z^{n}$, the study of groups of intermediate growth, and the study around Gromov's problem on the existence of groups of exponential but not uniformly exponential growth [Gro81b]. This paper describes the main developments of the second and third directions in detail, while the direction of study of growth series is only briefly mentioned towards the end of Section 3.

It was discovered that for many groups and even for classes of groups, the function represented by the growth series $\Gamma(z)$ is rational. For instance, this holds for virtually abelian groups [Kla81a, Kla81b, Ben83], for Gromov hyperbolic groups (with any finite system of generators) [Gro87, CDP90a], and for Coxeter groups with the canonical system of generators [Par91]. But the growth series can be irrational even in the case of nilpotent groups of nilpotency degree 2 (for instance, for some higher rank Heisenberg groups), and it can be rational for one system of generators and irrational for another [Sto96]. A very interesting approach to the study of growth functions, together with applications, is suggested by Kyoji Saito [Sai10, Sai11].

All known examples of groups of intermediate growth are groups of branch type and are self-similar groups, or have some self-similarity features (for example, groups from the class $\mathcal{G}_{\omega}, \omega \in \Omega$ discussed in section 9 ), or arise from constructions based on the use of branch groups of self-similar type. These groups act on spherically homogeneous rooted trees, and can be studied using techniques which have been developed during the last three decades. 
Until recently, there were no examples of groups of intermediate growth with a precise estimate on the growth rate (there were only upper and lower bounds on the growth as discussed in detail in section 11). Results about oscillatory behavior of the growth function were presented in [Gri84b] and [Gri85b] in connection with the existence of groups with incomparable growth. Recent articles of L. Bartholdi and A. Erschler [BA10, BE11] provide examples of groups with explicitly computed intermediate behavior of the type $\exp n^{\alpha}, \alpha<1$ and many other types of growth, as given in Theorem 13.3.

During the last three decades, many remarkable properties of groups of intermediate growth were found, and some of these properties are listed below. But unfortunately, we do not know yet if there are finitely presented groups of intermediate growth, and this is the main open problem in the field.

The paper is organized as follows. After providing some background about group growth in section 3, and formulation of Milnor's Problem in section 4, we discuss geometric motivations for the group growth and the relation of growth with amenability (sections 5 and 7). Section 6 describes results obtained before 1981 and section 8 contains an account of results about the polynomial growth case.

We describe the main construction of groups of intermediate growth, methods of obtaining upper and lower bounds, and the Bartholdi-Erschler construction in sections 9, 11 and 13). The Gap Conjecture is the subject of section 10. In section 12, we discuss the relation between the growth of groups and asymptotic characteristics of random walks on them. The final section 14 contains a short discussion concerning Gromov's Problem on groups of uniformly exponential growth, on the oscillation phenomenon that holds in the intermediate growth case, and on the role of just-infinite groups in the study of group growth.

A number of open problems are included in the text. In our opinion they are among the most important problems in the field of group growth. We hope that these problems will stimulate further studies of group growth and related topics.

During the last two decades, a number of nice expositions of various topics related to the growth of groups and their application have been published. These include the following sources [Wag93, Nav11, GdlH97, CSMS01, GP08]. We especially recommend the book of P. de la Harpe [dlH00], which is a comprehensive source of information about finitely generated groups in general and, in particular, about their growth, as well as the recent books of T. Ceccherini-Silberstein and M. Coornaert [CSC10] and A. Mann [Man12] which contains additional material.

The theory of growth of groups is a part of a bigger area of mathematics that studies coarse asymptotic properties of various algebraic and geometric objects. Some views in this direction can be found in the following sources: [Lub94, BH99, Roe03, BdlHV08, NY12].

The above sources are recommended to the reader for an introduction to the subject, to learn more details about some of the topics considered in this article, or to get information about some other directions of research involving the growth of groups.

\section{ACKNowledgments}

The author would like to express his thanks to L. Bartholdi, A. Bonifant, T. CheccheriniSilberstein, T. Delzant, A. Erschler, V. Kaimanovich, A. Mann, T. Nagnibeda, P. de la Harpe, I. Pak, M. Sapir, and J.S. Wilson. My special thanks to S. Sutherland and Z. Sunic for tremendous help with preparation of this paper and for valuable remarks, comments and 
suggestions. Parts of this work were completed during a visit to the Institut Mittag-Leffler (Djursholm, Sweden) during the program Geometric and Analytic Aspects of Group Theory.

\section{Preliminary facts}

Let $G$ be a finitely generated group with a system of generators $A=\left\{a_{1}, a_{2}, \ldots, a_{m}\right\}$ (throughout the paper we consider only infinite finitely generated groups and only finite systems of generators). The length $|g|=|g|_{A}$ of the element $g \in G$ with respect to $A$ is the length $n$ of the shortest presentation of $g$ in the form

$$
g=a_{i_{1}}^{ \pm 1} a_{i_{2}}^{ \pm 1} \ldots a_{i_{n}}^{ \pm 1}
$$

where $a_{i_{j}}$ are elements in $A$. This depends on the set of generators, but for any two systems of generators $A$ and $B$ there is a constant $C \in \mathbb{N}$ such that the inequalities

$$
|g|_{A} \leq C|g|_{B}, \quad|g|_{B} \leq C|g|_{A} .
$$

hold. To justify this it is enough to express each $a$-generator as a word in $b$-generators and vice versa: $a_{i}=A_{i}\left(b_{\mu}\right), b_{j}=B_{j}\left(a_{\nu}\right)$. Then $C=\max _{i, j}\left\{|A|_{i},|B|_{j}\right\}$, where $|W|$ denotes the length of the word $W$. In addition to the length, one can also introduce a word metric $d_{A}(g, h)=\left|g^{-1} h\right|, g, h \in G$ on a group which is left invariant. More general type of leftinvariant metrics and length functions on groups can be considered and studied as well. For instance, one can assign different positive weights to generators and define the length and metric according to these weights.

The growth function of a group $G$ with respect to the generating set $A$ is the function

$$
\gamma_{G}^{A}(n)=\left|\left\{g \in G:|g|_{A} \leq n\right\}\right|,
$$

where $|E|$ denotes the cardinality of the set $E$, and $n$ is a natural number.

If $\Gamma=\Gamma(G, A)$ is the Cayley graph of a group $G$ with respect to a generating set $A$, then $|g|$ is the combinatorial distance from vertex $g$ to vertex $e$ (represented by identity element $e \in G$ ), and $\gamma_{G}^{A}(n)$ counts the number of vertices at combinatorial distance $\leq n$ from $e$ (i.e., it counts the number of elements in the ball of radius $n$ with center at the identity element).

It follows from (3.1) that the growth functions $\gamma_{G}^{A}(n), \gamma_{G}^{B}(n)$ satisfy the inequalities

$$
\gamma_{G}^{A}(n) \leq \gamma_{G}^{B}(C n), \quad \gamma_{G}^{B}(n) \leq \gamma_{G}^{A}(C n) .
$$

The dependence of the growth function on the generating set is an inconvenience and it is customary to avoid it by using the following trick. Following J. Milnor, two functions on the naturals $\gamma_{1}(n)$ and $\gamma_{2}(n)$ are called equivalent (written $\gamma_{1}(n) \sim \gamma_{2}(n)$ ) if there is a constant $C \in \mathbb{N}$ such that $\gamma_{1}(n) \leq \gamma_{2}(C n), \gamma_{2}(n) \leq \gamma_{1}(C n)$ for all $n \geq 1$. Then according to (3.2), the growth functions constructed with respect to two different systems of generators are equivalent. The class of equivalence $\left[\gamma_{G}^{A}(n)\right]$ of the growth function is called the degree of growth (the growth degree), or the rate of growth of a group $G$. It is an invariant of a group not only up to isomorphism but also up to a weaker equivalence relation quasi-isometry.

Recall that two finitely generated groups $G$ and $H$ with generating sets $A$ and $B$ respectively are quasi-isometric if the metric spaces $\left(G, d_{A}\right),\left(H, d_{B}\right)$ are quasi-isometric. Here $d_{A}$ and $d_{B}$ are the word metrics on $G$ and $H$ defined as $d_{A}(f, g)=\left|f^{-1} g\right|_{A}, d_{B}(h, l)=\left|h^{-1} l\right|_{B}$, with $f, g \in G$ and $h, l \in H$. Two metric spaces $\left(X, d_{1}\right),\left(Y, d_{2}\right)$ are quasi-isometric if there is a map $\phi: X \rightarrow Y$ and constants $C \geq 1, D \geq 0$ such that

$$
\frac{1}{C} d_{1}\left(x_{1}, x_{2}\right)-D \leq d_{2}\left(\phi\left(x_{1}\right), \phi\left(x_{2}\right)\right) \leq C d_{1}\left(x_{1}, x_{2}\right)+D
$$


for all $x_{1}, x_{2} \in X$; a further requirement is that there is a constant $L>0$ so that for any point $y \in Y$, there is a point $x \in X$ with

$$
d_{2}(y, \phi(x)) \leq L
$$

This concept is due to M. Gromov [Gro87] and is one of the most important notions in geometric group theory, allowing the study of groups from coarse point of view.

It is easy to see that the growth of a group coincides with the growth of a subgroup of finite index, and that the growth of a group is not smaller than the growth of a finitely generated subgroup or a factor group. We will say that a group is virtually nilpotent (resp. virtually solvable) if it contains a nilpotent (solvable) subgroup of finite index.

We will also consider a preoder $\preceq$ on the set of growth functions:

$$
\gamma_{1}(n) \preceq \gamma_{2}(n)
$$

if there is an integer $C>1$ such that $\gamma_{1}(n) \leq \gamma_{2}(C n)$ for all $n \geq 1$. This makes a set $\mathcal{W}$ of growth degrees of finitely generated groups a partially ordered set. The notation $\prec$ will be used in this article to indicate a strict inequality.

Observe that Schwarz in his note [Š 55] used formally weaker equivalence relation $\sim_{1}$ given by inequalities

$$
\gamma_{G}^{A}(n) \leq C \gamma_{G}^{B}(C n), \quad \gamma_{G}^{B}(n) \leq C \gamma_{G}^{A}(C n) .
$$

But indeed both equivalence relations coincide when restricted to the set of growth functions of infinite finitely generated groups, as was observed in [Gri84b, Proposition3.1]. Therefore we will use either of them depending on the situation.

Because of the independence of the growth rate on a generating set, we will usually omit subscripts in the notation.

Let us list the main examples of growth rates that will be used later.

- The power functions $n^{\alpha}$ belong to different equivalence classes for different $\alpha \geq 0$.

- The polynomial function $P_{d}(n)=c_{d} n^{d}+\cdots+c_{1} n+c_{0}$, where $c_{d} \neq 0$ is equivalent to the power function $n^{d}$.

- All exponential functions $\lambda^{n}, \lambda>1$ are equivalent and belong to the class $\left[2^{n}\right]$ (or to $\left[e^{n}\right]$.)

- All functions of intermediate type $e^{n^{\alpha}}, 0<\alpha<1$ belong to different equivalence classes.

Observe that this is not a complete list of rates of growth that a group may have.

A free group $F_{m}$ of rank $m$ has $2 m(2 m-1)^{n-1}$ elements of length $n$ with respect to any free system of generators $A$ and

$$
\gamma_{F_{m}}^{A}(n)=1+2 m+2 m(2 m-1)+\cdots+2 m(2 m-1)^{n-1} \sim 2^{n} .
$$

Since a group with $m$ generators can be presented as a quotient group of a free group of rank $m$, the growth of a finitely generated group cannot be faster than exponential (i.e., it can not be superexponential). Therefore we can split the growth types into three classes:

- Polynomial growth. A group $G$ has a polynomial growth is there are constants $C>0$ and $d>0$ such that $\gamma(n)<C n^{d}$ for all $n \geq 1$. This is equivalent to

$$
\overline{\lim }_{n \rightarrow \infty} \frac{\log \gamma(n)}{\log n}<\infty .
$$


A group $G$ has weakly polynomial growth if for some $d \in(0, \infty)$

$$
\underline{\lim }_{n \rightarrow \infty} \frac{\log \gamma(n)}{\log n}<\infty
$$

(as we will see later (3.5) is equivalent to (3.6)).

- Intermediate growth. A group $G$ has intermediate growth if $\gamma(n)$ grows faster than any polynomial but slower than any exponent function $\lambda^{n}, \lambda>1$ (i.e. $\left.\gamma(n) \prec e^{n}\right)$.

- Exponential growth. A group $G$ has exponential growth if $\gamma(n)$ is equivalent to $2^{n}$.

The case of exponential growth can be redefined in the following way. Because of the obvious semi-multiplicativity

$$
\gamma(m+n) \leq \gamma(m) \gamma(n)
$$

the limit

$$
\lim _{n \rightarrow \infty} \sqrt[n]{\gamma(n)}=\kappa
$$

exists. If $\kappa>1$ then the growth is exponential. If $\kappa=1$, then the growth is subexponential and therefore is either polynomial or intermediate.

Remark 3.1. In some situations it is reasonable to extend the domain of growth function to all nonnegative real numbers. This can be done, for instance, by setting $\gamma(x)=\gamma([x])$, where $[x]$ is the integer part of $x$.

Milnor's equivalence relation $\sim$ on the set of growth functions is a coarse approach to the study of growth. Sometimes, in order to study the growth, weaker equivalence relations on the set of monotone functions are used, for instance when the factor $C$ appears not only in the argument of the growth function but also in front of it (as in the case of the Schwarz equivalence relation). Additionally, in some situations, an additive term appears in the form $C \gamma(C n)+C n$ (for instance in the study of Dehn functions of finitely presented groups, as suggested by Gromov [Gro87]). Instead of the constant $C$ one can use the equivalence relation with $C=C(n)$, where $C(n)$ is a slowly growing function, for instance a polynomial. In contrast with the suggested ways of weakening the equivalence relation leading to a loss of some information about the growth, the more precise evaluation of the rate of growth requires tools from analysis based on classical asymptotic methods.

There is a standard way to associate a growth series to a growth function, defined by

$$
\Gamma(z)=\sum_{n=0}^{\infty} \gamma(n) z^{n} .
$$

The radius of converges of the series is $R=1 / \kappa \geq 1$. If the analytic function represented by this series is a rational function, or more generally an algebraic function, then the coefficients $\gamma(n)$ grow either polynomially or exponentially and therefore the intermediate growth is impossible in this case. For some classes of groups (e.g., abelian groups or Gromov hyperbolic groups), the growth series is always a rational function [Can80, Gro87]. There are groups or classes of groups for which there is a system of generators with rational or algebraic growth series; for instance, Coxeter groups have this property with respect to the canonical system of generators [Par91]. There are examples of groups for which the growth series is a rational function for one system of generators but is not rational for another system of generators [Sto96]. Typically the growth series of a group is a transcendental function. 
There is also interest in the study of the complete growth function

$$
\Gamma_{*}(z)=\sum_{g \in G} g z^{|g|} \in \mathbb{Z}[G][[z]]
$$

viewed an element of the ring of formal power series with coefficients in a group ring $\mathbb{Z}[G]$ of a group, or an operator growth function

$$
\Gamma_{\pi}(z)=\sum_{g \in G} \pi(g) z^{|g|},
$$

where $\pi(g)$ is a representation of the group in a Hilbert space (or more generally, a Banach space) [Lia96, GN97]. An important case is when $\pi$ is a left regular representation of the group in $l^{2}(G)$.

Remark 3.2. The growth can be defined for many algebraic, combinatorial, geometric, probabilistic and dynamical objects. For instance, one can speak about the growth of a connected locally finite graph $\Gamma$, by which we mean the growth of the function $\gamma_{\Gamma, v}(n)$, which counts the number of vertices at combinatorial distance $\leq n$ from a base vertex $v$. Growth of such graphs can be superexponential, but if the graph has uniformly bounded degree (for instance is a regular graph), the growth is at most exponential. The growth does not depend on the choice of $v$.

The growth of a Riemannian manifold $M$ is, by definition, the growth as $r \rightarrow \infty$ of the function $\gamma(r)=\operatorname{Vol}\left(B_{x}(r)\right)$ expressing the volume of a ball of radius $r$ with center at fixed point $x \in M$. The rate of growth is independent of the choice of $x$.

The definition of growth of a semigroup is similar to the group case. Also one can define growth of finitely generated associative algebras, graded associative algebras, Lie algebras, etc. For instance, if $\mathcal{A}=\bigoplus_{n=0}^{\infty} \mathcal{A}_{n}$ is a finitely generated associative graded algebra defined over a field $\mathbb{F}$ then the growth of dimensions $d_{n}=\operatorname{dim}_{\mathbb{F}} \mathcal{A}_{n}$ determines the growth of $\mathcal{A}_{n}$ and the corresponding growth series

$$
\mathcal{H}(z)=\sum_{n=0}^{\infty} d_{n} z^{n}
$$

usually is called a Hilbert-Poincare series.

Given a countable group $G$ and a probabilistic measure $\mu$ on it whose support generates $G$, one can consider a right random walk on $G$ which begins at the identity element $e \in G$ and such that the transitions $g \rightarrow g h$ happen with probability $\mu(h)$. One of the main characteristics of such random process is the probability $P_{e, e}^{(n)}$ of return after $n$ steps. This probability may decay exponentially $\left(\right.$ as $r^{n}, r<1$ ) or subexponentially. The value

$$
r=\overline{\lim }_{n \rightarrow \infty} \sqrt[n]{P_{e, e}^{(n)}}
$$

is called the spectral radius of the random walk (observe that $\lim _{n \rightarrow \infty} \sqrt[2 n]{P_{e, e}^{(2 n)}}$ exists). It was introduced by H. Kesten in [Kes59b]. In the case of a symmetric measure (i.e. when for any $g \in G$, the equality $\mu(g)=\mu\left(g^{-1}\right)$ holds), the spectral radius coincides with the norm of the Markov operator of the random walk, and the subexponential decay of $P_{e, e}^{(n)}$ (i.e. the equality $r=1$ ) holds if and only if the group is amenable in the von Neumann sense (i.e. when $G$ has a left invariant mean) [vN29, Kes59a]. We will discuss this topic in more details later in this paper. 


\section{The Problem and the Conjecture of Milnor}

In his note published in the American Mathematical Monthly [Mil68b], Milnor formulated a remarkable problem concerning the growth of groups, as well as an ingenious conjecture concerning the polynomial growth case. We reproduce them here, but dividing the problem into two parts (which was not done in [Mil68b]). As before, $\gamma(n)$ denotes the growth function of a finitely generated group (we shall keep this notation through the paper).

\section{Milnor's Problem.}

(I) Is the function $\gamma(n)$ necessarily equivalent either to a power of $n$ or to exponential function $2^{n}$ ?

(II) In particular, is the growth exponent

$$
d=\lim _{n \rightarrow \infty} \frac{\log \gamma(n)}{\log n}
$$

always either a well-defined integer or infinity? For which groups is $d<\infty$ ?

Milnor's Conjecture. (A possible conjecture would be that $d<\infty$ if and only if $G$ contains a nilpotent subgroup of finite index).

The first part of Milnor's Problem is a question on the existence of groups of intermediate growth. The second part and the conjecture are oriented toward the study of the polynomial growth case and the regularity properties of growth functions.

Clearly the motivation for suggesting such a problem and a conjecture was based on Milnor's background in the area of group growth as of 1968. Below we will provide more information on what was known about group growth around 1968.

In short, the history of solutions of Milnor's Problem and his Conjecture is the following. The Conjecture was confirmed by M. Gromov [Gro81a]. This, together with the results of Guivarc'h [Gui70, Gui71], B. Hartley (unpublished, but see "Added in Proof" in [Wol68]), and H. Bass [Bas72], showed that the upper limit (3.5) is a non-negative integer and that the finiteness of the limit (4.1) implies that the group is virtually nilpotent. The existence of the limit (4.1) also follows from results of the mathematicians quoted above, giving the complete solution of the second part of Milnor's Problem. In the case of nilpotent groups, the existence of the limit

$$
\lim _{n \rightarrow \infty} \frac{\gamma(n)}{n^{d}}
$$

(where $d$ is the degree of polynomial growth) was proved by P. Pansu [Pan83] and is an additional bonus in the study of the polynomial growth case.

The first part of Milnor's Problem was solved in the negative by the author in 1983 [Gri83, Gri84b, Gri85a]. It was shown that there are groups of intermediate growth, and moreover that there are uncountably many of them. Also, it was shown that there are pairs of groups with incomparable growth in the sense of the order $\preceq$. In addition, many other results about growth and algebraic properties of groups of intermediate growth were obtained around 1984 and later. Despite a negative solution of the first part of Milnor's problem, the fact that there are groups of intermediate growth made group theory richer and substantially extended the area of its applications.

All known examples of groups of intermediate growth are infinitely presented groups. 
Problem 1. Is it true that the growth function of a finitely presented group is equivalent either to a polynomial or to the exponential function $2^{n}$ ?

In [GP08], it is conjectured that there are no finitely presented groups of intermediate growth. At the same time, the author suggests even a stronger conjecture.

Conjecture 1. A finitely presented group either contains a free subsemigroup on two generators or is virtually nilpotent.

Problem 1 is the main remaining open problem concerning group growth. As we will see later, there are many recursively presented groups of intermediate growth, in particular, the groups $\mathcal{G}=\mathcal{G}_{\xi}$ and $\mathcal{G}_{\eta}$ described in section 9 are recursively presented; we note that the group $\mathcal{G}$ will serve in this text as the main illustrating example. By Higman's embedding theorem [LS77] such groups embed into finitely presented groups. It is worth mentioning that for the group $\mathcal{G}_{\xi}$, there is a very precise and nice embedding based on the use of Lysionok presentation (9.1), as was observed in [Gri98] (a similar claim holds for $\mathcal{G}_{\eta}$ ). In fact, a similar embedding exists for all groups with finite $L$-presentation that are defined and studied in [BGŠ03, Bar03a]. The corresponding finitely presented group (which we denote here $\tilde{\mathcal{G}}_{\xi}$ ) is an ascending $H N N$-extension of $\mathcal{G}_{\xi}$. It has a normal subgroup $N$ which is ascending

union of conjugates of a subgroup isomorphic to $\mathcal{G}_{\xi}$ with the quotient $\tilde{\mathcal{G}}_{\xi} / N$ isomorphic to a infinite cyclic group. Unfortunately (or fortunately), the group $\tilde{\mathcal{G}}_{\xi}$ has exponential growth but shares the property of amenability with $\mathcal{G}_{\xi}$. The quotients of this group are described in [SW02]. The idea of finding a finitely presented group containing $\mathcal{G}_{\xi}$ as a normal subgroup (with the hope of thus obtaining a finitely presented group of intermediate growth) fails, as was observed by M. Sapir, because $\tilde{\mathcal{G}}_{\xi}$ cannot serve as a normal subgroup of any finitely presented groups (see the argument in http://mathoverflow.net/questions/ 73076/higman-embedding-theorem/).

Moreover, it was observed by P. de la Harpe and the author that any finitely presented group $\hat{\mathcal{G}}$ that can be homomorphically mapped onto $\mathcal{G}$ contains a free subgroup on two generators and hence is of exponential growth. In view of these facts it would be interesting to find a finitely presented group with a normal subgroup of intermediate growth and to find a finitely presented group without a free subgroup on two generators (or perhaps even a finitely presented amenable group) that can be mapped onto a group of intermediate growth. This is discussed in detail in [BGH13].

For the group $\mathcal{G}_{\eta}$ and many other groups possessing a presentation of the type (9.1) (i.e. a presentation involving a finite set of relators and their iterations by one or more, but finitely many, substitutions), embeddings into finitely presented groups similar to the one for $\mathcal{G}$ also exist [BGŠ03, Bar03a].

\section{Relations Between group GROWTH And Riemannian GeOMEtRy}

One of the first results showing the usefulness of the notion of group growth was the result of A.S. Schwarz, who proved the following theorem in 1957.

Theorem 5.1. Let $\tilde{M}$ be the universal cover of a compact Riemannian manifold $M$. Then the rate of growth of $\tilde{M}$ is equal to the rate of growth of the fundamental group $\pi_{1}(M)$.

In addition to the obvious examples of groups with polynomial or exponential growth (free abelian groups and free noncommutative groups respectively), in his note Schwarz produced 
examples of solvable (and even metabelian) groups of exponential growth. These are defined as a semidirect product of $\mathbb{Z}^{d}, d \geq 2$ and a cyclic group generated by an automorphism $\varphi \in S L_{d}(\mathbb{Z})$ given by a matrix with at least one eigenvalue off the unit circle.

In theorem 5.1, the comparison of the growth rates of a manifold and a group is considered with respect to the equivalence relation

$$
\gamma_{1}(n) \sim_{1} \gamma_{2}(n) \quad \Leftrightarrow \quad \exists C>0 \forall n \quad \gamma_{1}(n) \geq C \gamma_{2}(C n) \& \gamma_{2}(n) \geq C \gamma_{1}(C n) ;
$$

the domain of $\gamma_{\pi_{1}(M)}(n)$ is extended in the natural way to $\mathbb{R}_{+}$. Of course, the equivalence relations $\sim$ and $\sim_{1}$ defined on the set of monotone functions of natural argument are different. But, as was already mentioned in the previous section, they coincide on the set of growth functions of finitely-generated infinite groups.

The theorem of Schwarz relates the growth of groups with the volume growth of the universal cover of a compact Riemannian manifold. In fact a more general statement which deals with non-universal and even non-regular coverings holds; we formulate this below.

Milnor's investigation into the relation between growth and curvature led him to the following two results [Mil68a].

Theorem 5.2. If $M$ is a complete d-dimensional Riemannian manifold whose mean curvature tensor $R_{i j}$ is everywhere positive semi-definite, then the growth function $\gamma(n)$ associated with any finitely generated subgroup of the fundamental group $\pi_{1}(M)$ must satisfy $\gamma(n)<C n^{d}$ for some positive constant $C$.

Theorem 5.3. If $M$ is a compact Riemannian manifold with all sectional curvatures less than zero, then the growth function of the fundamental group $\pi_{1}(M)$ is exponential: $\gamma(n)>a^{n}$ for some constant $a>1$.

The next example considered by Milnor was the first step in the direction toward understanding the growth of nilpotent groups. Let $G$ be the nilpotent Lie group consisting of all $3 \times 3$ triangular real matrices with 1 's on the diagonal, and let $\mathcal{H}_{3}$ be the subgroup consisting of all integer matrices of the same form. Then the coset space $G / \mathcal{H}_{3}$ is a compact 3-dimensional manifold with fundamental group $\mathcal{H}_{3}$.

Lemma 5.4. The growth function of $\mathcal{H}_{3}$ is quartic:

$$
C_{1} n^{4}<\gamma_{\mathcal{H}_{3}}(n)<C_{2} n^{4}
$$

with $0<C_{1}<C_{2}$.

Corollary 5.5. No Riemannian metric on $G / \mathcal{H}_{3}$ can satisfy either the hypothesis of Theorem 5.2 or the hypothesis of Theorem 5.3.

The ideas of Milnor were used by A. Aves [Ave70] to get a partial answer to a conjecture of E. Hopf [Hop48]: Let M be a compact, connected Riemannian manifold without focal points. Then either the fundamental group $\pi_{1}(M)$ has exponential growth, or $\mathrm{M}$ is flat.

Now let us go back to the theorem of Schwarz and present a more general statement. But before that, we need to recall some notions from geometric group theory. Let $G$ be a finitely generated group with a system of generators $A$ and let $H<G$ be a subgroup. Let $\Gamma=\Gamma(G, H, A)$ be the Schreier graph determined by the triple $(G, H, A)$. The vertices of $\Gamma$ are in bijection with cosets $g H, g \in G$, and two vertices $g H$ and $h H$ are joined by oriented edge (labeled by $a \in A$ ) if $h H=a g H$. This notion is a generalization of the notion of a 
Cayley graph of a group (Cayley graphs correspond to the case when $H=\{e\}) . \Gamma(G, H, A)$ is a $2 m$-regular graph, where $m$ is the cardinality of the generating set. As was already defined, the growth function $\gamma_{\Gamma}(n)$ of a graph counts the number of vertices at combinatorial distance $\leq n$ from the base vertex $v$ (we remind the reader that the choice of $v$ does not play a role; it is natural for a Schreier graph to choose $v=1 H$ ).

If a group $G$ with a generating set $A$ acts transitively on a set $X$ then $\Gamma(G, H, A)$ is isomorphic to the graph of the action, i.e. the graph $\Gamma_{*}$ with set of vertices $X$ and set of edges consisting of pairs $(x, a(x)), x \in X, a \in A$. In this case the subgroup $H$ coincides with the stabilizer $s t_{G}(x)$.

Theorem 5.6. Let $M$ be a compact Riemannian manifold, let $H$ be a subgroup of the fundamental group $\pi_{1}(M)$ and let $\tilde{M}$ be a cover of $M$ corresponding to $H$ supplied by a Riemannian metric lifted from $M$. Then the growth function $\gamma_{\tilde{M}}(r)$ is $\sim_{1}$ equivalent to the growth function $\gamma_{\Gamma\left(\pi_{1}(M), H, A\right)}(r)$ (with domain naturally extended to $\mathbb{R}_{+}$) of the Schreier graph $\Gamma\left(\pi_{1}(M), H, A\right)$ where $A$ is a finite system of generators of $\pi_{1}(M)$.

Proof. Triangulate $M$, lift the triangulation to $\tilde{M}$, and make the comparison of the volume growth of $\tilde{M}$ with the growth function $\gamma_{\Gamma\left(\pi_{1}(M), H, A\right)}(r)$ using the graph of the action of $\pi_{1}(M)$ on the preimage $p^{-1}(x)$, where $p: \tilde{M} \rightarrow M$ is the canonical projection, and then apply the arguments from [క̌ 55$]$.

A particular case of this theorem mentioned in [Gri89b] is the case of a regular (i.e. Galois) cover, i.e. when $H$ is a normal subgroup of $\pi_{1}(M)$. In this case, the growth of the covering manifold $\tilde{M}$ coincides with the growth of the quotient group $\pi_{1}(M) / H$ and the latter is isomorphic to the group of deck transformations of the cover. This fact together with the results about groups of intermediate growth obtained in [Gri84b] allowed the author to construct uncountably many Riemannian surfaces supplied with groups of isometries acting on them cocompactly, having a topological type of oriented surface of infinite genus with one end, and which are not pairwise quasi-isometric [Gri89b].

\section{Results about group growth obtained Before 1981}

Among the first publications to use of the notion of group growth were [Š55] and [AVŠ57]. The result of the article of Adelson-Velskii and Shreider relates growth with amenability and will be discussed in the next section. Then after more than a decade of sporadic appearances of group growth in various articles (partly listed in the introduction), the papers of Milnor [Mil68a, Mil68c] and his note [Mil68b] appeared.

These publications, followed by Wolf's article [Wol68], attracted attention of many researchers and a flurry of activity occurred during a short period. The fact that nilpotent groups have polynomial growth was already observed in [Mil68a] (the example of Heisenberg group) and in full generality was studied by Wolf [Wol68] and B. Hartly (see "Added in Proof" in [Wol68]), Guivarc'h [Gui70, Gui71] and by H. Bass [Bas72], who showed that for nilpotent groups, the growth function satisfies the inequalities

$$
C_{1} n^{d} \leq \gamma(n) \leq C_{2} n^{d} .
$$

Here

$$
d=\sum_{i} i \cdot \operatorname{rank}_{\mathbb{Q}}\left(\gamma_{i}(G) / \gamma_{i+1}(G)\right),
$$


where $\gamma_{i}(G)$ is $i$ th member of the lower central series of the group, $C_{1}$ and $C_{2}$ are positive constants, and $\operatorname{rank}_{\mathbb{Q}}(A)$ is the torsion free rank of the abelian group $A$.

In [Wol68], Wolf proved that a polycyclic group either contains a nilpotent subgroup of finite index and has polynomial growth or the growth is exponential. At the same time, Milnor observed that a solvable but not polyciclic group has exponential growth. This, in combination with Wolf's result, led to the fact that the growth of a solvable group is exponential except for the case when the group is virtually nilpotent.

A self-contained proof of the main result about growth of solvable groups was given by Tits in the appendix to Gromov's paper [Gro81a]. In [Ros74] J. Rosenblatt showed that groups of subexponential growth are superamenable (the class of superamenable groups is a subclass of the class of amenable groups, for the definition see the next section), and indicated that a solvable group of exponential growth contains a free subsemigroup on two generators (perhaps the latter was known before, but the author has no corresponding reference).

The remarkable theorem of Tits (usually called the Tits Alternative) implies that a finitely generated subgroup of a linear group (i.e. a subgroup of $G L_{n}(\mathbb{F}), n \geq 1, \mathbb{F}$ a field), either contains a free subgroup on two generators or is virtually solvable [Tit72]. This, together with the results about the growth of solvable groups, imply that the growth of a finitely generated linear group is either polynomial or exponential.

The first part of Milnor's problem on growth was included by S.I. Adian in his monograph [Adi79], dedicated to the one of the most famous problems in Algebra - the Burnside Problem on periodic groups. Adian showed that the free Burnside group

$$
B(m, n)=\left\langle a_{1}, a_{2}, \ldots, a_{m} \mid X^{n}=1\right\rangle,
$$

of exponent $n \geq 665$ ( $n$ odd ) with $m \geq 2$ has exponential growth. This is a stronger result than the result of P.S. Novikov and S.I. Adian [NA68b] about the infiniteness of $B(m, n)$ in the case $m \geq 2$ and odd $n \geq 4381$. A number of results about growth of semigroups was obtained by V. Trofimov [Tro80] (for more recent developments about growth of semigroups see [BRS06, Shn04, Shn05]). However, we are not going to get much into the details of growth in the semigroup case.

A useful fact about groups of intermediate growth is due to S. Rosset [Ros76].

Theorem 6.1. If $G$ is a finitely generated group which does not grow exponentially and $H$ is a normal subgroup such that $G / H$ is solvable, then $H$ is finitely generated.

The basic tool for the proof of this theorem is Milnor's lemma.

Lemma 6.2. If $G$ is a finitely generated group with subexponential growth, and if $x, y \in G$, then the group generated by the set of conjugates $y, x y x^{-1}, x^{2} y x^{-2}, \ldots$ is finitely generated.

The theorem of Rosset was stated in 1976 for groups of subexponential growth but its real application is to the case of groups of intermediate growth, because by a theorem of Gromov the groups of polynomial growth are virtually nilpotent and all subgroups in such groups are finitely generated.

The theorem of Rosset can be generalized. In the next statement we use the notion of elementary amenable group which is defined in the next section. Observe that solvable groups constitute a subclass of the class of elementary amenable groups. 
Theorem 6.3. Let $G$ be a finitely generated group with no free subsemigroups on two generators and let the quotient $G / N$ be an elementary amenable group. Then the kernel $N$ is a finitely generated group.

The proof of this fact is based on the use of a version of Milnor's lemma (Lem. 1 from [LMR95]) and transfinite induction on the "complexity" of elementary amenable groups defined below. Observe that this induction was used by Chou to prove the absence of groups of intermediate growth, infinite finitely generated torsion groups, and infinite finitely generated simple groups in the class of elementary amenable groups [Cho80]. Interesting results about algebraic properties of "generalized" elementary groups are obtained by D. Osin [Osi02].

In the introduction to his paper published in 1984, [Gri84b] the author wrote: "In the past decade, in group theory there appeared a direction that could be called 'Asymptotic Group Theory' " (perhaps this was the first time when the name "asymptotic group theory" was used in the mathematical literature). Milnor is one of the pioneers of this direction and his ideas and results contributed a lot to its formation. Asymptotic group theory studies various asymptotic invariants of groups, first of all asymptotic characteristics of groups (like growth), many of which were defined and studied during the last three decades. For instance, the notion of cogrowth (or relative growth) was introduced by author in [Gri80a], and later was used by him, Olshanskii and Adian to answer some questions related to the von Neumann Conjecture on non-amenable groups [Gri79, $\mathrm{Ol}^{\prime} 80$, Adi82]. The notion of subgroup growth was introduced by Grunewald, Segal and Smith in [GSS88] and studied by many mathematicians (see [LS03] and the literature cited there). Dehn functions and their growth were introduced by Gromov and also happen to be a popular subject for investigation (see [BH99] and citations therein). Many asymptotic invariants of groups were introduced and studied by Gromov in [Gro93], and many other asymptotic invariants has been introduced since then. The asymptotic methods in the case of algebras (including the topics of self-similarity) are discussed in survey of E. Zelmanov [Zel05, Zel07] and in [Gro08]. The direction of asymptotic group theory is flourishing at present time and the author has no doubt that the situation will not change in the next few decades.

\section{Growth and Amenability}

Definition 7.1 (John Von Neumann (1929)). A group $G$ is called amenable, if there is a finitely additive measure $\mu$ defined on the algebra of all subsets of $G$ and such that:

- $\mu(G)=1,0 \leq \mu(E) \leq 1, \forall E \subset G$

- $\mu$ is left invariant, i.e. $\forall E \subset G$ :

$$
\mu(E)=\mu(g E), \quad \forall g \in G, \forall E \subset G .
$$

The measure $\mu$ determines an invariant, positive, normalized functional $m$ on Banach space $l^{\infty}(G)$ defined by

$$
m(f)=\int_{G} f d \mu
$$

and is called a left invariant mean (LIM). And vice versa, any invariant, positive, normalized functional $m$ determines a measure $\mu$ by restriction of its values on the characteristic functions of subsets. In a similar way, von Neumann defined amenability of action of a group on a set [vN29]. 
The simplest example of a non-amenable group is the free group $F_{2}$ on two generators. The simplest examples of amenable groups are finite groups and commutative groups.

In the above definition the group $G$ is assumed to be a group with discrete topology. There is a version of this definition due to N.N. Bogolyubov [Bog], M. Day and others for general topological groups [HR79] but it depends on the choice of the space of functions (bounded continuous or bounded uniformly continuous functions) as was observed by P. de la Harpe [dlH73]. In the case of locally compact groups one can use any of the mentioned spaces to define amenability [HR79, Gre69]. Growth of groups also can be defined for locally compact compactly generated groups but we will not consider topological groups case here. We recommend to the reader the survey [dlHGCS99] on amenability and literature there.

Following M. Day [Day57], we use $A G$ to denote the class of amenable groups. This class is extremely important for various topics in mathematics. In this section we discuss briefly this notion because of its relation with growth. By the theorem of Adelson-Velskii and Schreider [AVŠ57], each finitely generated group of subexponential growth belongs to the class $A G$. This class contains finite groups and commutative groups and is closed under the following operations:

(1) taking a subgroup,

(2) taking a quotient group,

(3) extensions (i.e. an extension of amenable group by amenable group is amenable),

(4) directed unions

$G_{\alpha} \in A G, G_{\alpha} \subset G_{\beta}$ if $\alpha<\beta \Rightarrow \cup_{\alpha} G_{\alpha} \in A G$ (here we assume that the family of groups $\left\{G_{\alpha}\right\}$ is a directed family, i.e. for any $\alpha$ and $\beta$ there is $\gamma$ with $G_{\alpha} \leq G_{\gamma}$ and $\left.G_{\beta} \leq G_{\gamma}\right)$.

Let $E G$ be the class of elementary amenable groups. This is the smallest class of groups containing finite groups, commutative groups which is closed with respect to the operations (1)-(4). For such groups a kind of complexity can be defined in the following way, as was suggested by C. Chou [Cho80]. For each ordinal $\alpha$, define a subclass $E G_{\alpha}$ of $E G$. $E G_{0}$ consists of finite groups and commutative groups. If $\alpha$ is a limit ordinal then

$$
E G_{\alpha}=\bigcup_{\beta \preceq \alpha} E G_{\beta} .
$$

Further, $E G_{\alpha+1}$ is defined as the set of groups which are extensions of groups from the set $E G_{\alpha}$ by groups from the same set. The elementary complexity of a group $G \in E G$ is the smallest $\alpha$ such that $G \in E G_{\alpha}$.

One more class of subexponentially amenable groups, denoted here $S G$, was defined in [Gri98] as the smallest class which contains all groups of subexponential growth and is closed with respect to the operations (1)-(4). It is contained in the class of "good" groups, in the terminology of M. Freedman and P.Teichner [FT95]. Observe that the inclusions $E G \subset S G \subset A G$ hold. A general concept of elementary classes is developed by D. Osin [Osi02], along with an indication of their relation to the Kurosh-Chernikov classes. We will mention the class $S G$ again in section 12 .

The question of Day [Day57] concerning coincidence of the classes $A G$ and $E G$ was answered in the negative by the author as a result of the solution of the first part of Milnor's problem [Gri84b]. In fact, each group of intermediate growth belongs to the complement 
$A G \backslash E G$ as was showed in [Cho80]. And as there are uncountably many 2-generated groups of intermediate growth, the cardinality of the set $A G \backslash E G$ is the cardinality of the continuum.

The second question of Day about coincidence of classes $A G$ and $N F$ (the latter is the class of groups that do not contain a free subgroup on two generators) was answered in the negative by A. Olshanskii [Ol'80] and S. Adian [Adi82]. Sometimes this problem is formulated in the form of a conjecture, with attribution to von Neumann. A counterexample to a stronger version of the von Neumann conjecture was constructed in [Gri79], where a subgroup $H$ of a free group $F_{2}$ with the property that the action of $G$ on $G / H$ is nonamenable and some nonzero power of each element in $F_{2}$ belongs to some conjugate of $H$. All three articles [Gri79, Ol'80, Adi82] use the cogrowth criterion of amenability from [Gri80a].

It is important to mention that both problems of Day had negative solutions not only for the class of finitely generated groups but also for the class of finitely presented groups, as was established by the author and Olshanskii and Sapir respectively [Gri98, OS02]. In other words, there are examples of finitely presented amenable but non-elementary amenable groups, and there are examples of finitely presented non-amenable groups without a free subgroup on two generators. Therefore the situation with amenable/non-amenable groups is in a sense better than the situation with groups of intermediate growth where the existence of finitely presented groups is unknown (see problem 1).

The variety of different (but equivalent) definitions of the notion of amenable group is tremendous. Perhaps there is no any other notion in mathematics which may compete with the notion of amenable group in the number of different definitions. The criteria of amenability of Tarski, Fölner, Kesten, Reiter and many others were found during the eight decades of studies of amenability. Kesten's criterion expresses amenability in terms of spectral radius $r$ of random walk on a group in the following way: a group $G$ is amenable if and only if $r=1$ (here we should assume that the measure defining the random walk is symmetric and its support generate group, more on this in section 12).

In the final part of his article [Mil68a], Milnor raised the following question.

Consider a random walk on the fundamental group of compact manifold of negative curvature. Is the spectral radius $r$ necessarily less than 1 ?

In view of Kesten's criterion, this is equivalent to the question about non-amenability of the fundamental group of a compact manifold of negative curvature. This problem was positively solved by P. Eberlain [Ebe73]. Related results are obtained by Yau [Yau75] (see also Proposition 3 on page 98 of the book by Gromov, Pansu and Lafontaine [Gro81b]) and Chen [Che78]. As observed by T. Delzant, the solution of Milnor's problem 5.3 can also be deduced from the results and techniques of A. Avez paper [Ave70]. A theorem, due to M. Anderson, considers the case of non positive curvature (i.e. the curvature can be 0) [And87]. It is proved that amenable subgroup of such a group must be virtually abelian and must be the fundamental group of a flat totally geodesic submanifold. In fact, after the work of Gromov [Gro87] on hyperbolic groups, followed by the books [GH90, CDP90a], it became clear that fundamental groups of compact manifolds of negative curvature are nonelementary hyperbolic. Such groups contain a free subgroup on two generators and hence are nonamenable.

In 1974, J. Rosenblatt introduced the interesting notion of a superamenable group and proved that a group of subexponential growth is superamenable [Ros74]. A group $G$ is 
called superamenable if for any $G$-set $X$ and any nonempty subset $E \subset X$ there is a $G$ invariant finitely additive measure on the algebra of all subsets of $X$ taking values in $[0,+\infty)$ and normalized by condition $\mu(E)=1$. This property implies there is an invariant Radon measure for any cocompact continuous action on a locally compact Hausdorff space (private communication by N. Monod). It is worth mentioning that amenability of a group can be characterized by the property of a group having an invariant probability measure for any continuous action on a compact space, because of the theorem of Bogolyubov-Day (which generalizes a theorem of Bogolyubov-Krylov) [Gre69]. Superamenability is discussed in the book [Wag93]. In the next section we will mention it again.

\section{Polynomial growth}

Recall that a group $G$ has polynomial growth if there are constants $C>0$ and $d>0$ such that $\gamma(n)<C n^{d}$ for all $n \geq 1$, i.e. (3.5) holds. As was discussed in section 6 a nilpotent group has polynomial growth and therefore a virtually nilpotent group also has polynomial growth. In his remarkable paper [Gro81a], Gromov established the converse: polynomial growth implies the virtual nilpotence of the group.

Theorem 8.1. (Gromov 1981) If a finitely generated group $G$ has polynomial growth then $G$ contains a nilpotent subgroup of finite index.

This theorem, together with known information about growth of nilpotent groups, gives a wonderful characterization of groups of polynomial growth in algebraic terms: a finitely generated group has polynomial growth if and only if it is virtually nilpotent.

The proof of Gromov's theorem is very geometric by its nature, but it also uses some fundamental facts from algebra and analysis. Roughly speaking, the idea of Gromov consists of considering the group as a metric space, and at looking at this space via a "macroscope". The implementation of this idea goes through the development of the technique of limits of sequences of metric spaces. Given a group of polynomial growth $G$, this method assiociates with it a Lie group $\mathcal{L}_{\mathcal{G}}$ which has finitely many connected components, and a nontrivial homomorphism $\phi: G \rightarrow \mathcal{L}_{\mathcal{G}}$. The rest of the proof is a matter of mathematical culture modulo known facts from algebra and analysis including the Jordan theorem, Tits alternative and the Milnor-Wolf theorem on growth of solvable groups. The skeleton of Gromov's proof has been adapted to many other proofs. In particular, it initiated the study of asymptotic cones of groups [vdDW84, Gro93].

Combining his theorem with the results of Shub and Franks [Shu70] on properties of fundamental group of a compact manifold admitting an expanding self-covering, Gromov deduced the following statement.

Corollary 8.2. An expanding self-map of a compact manifold is topologically conjugate to an ultra-nil-endomorphism.

In fact Gromov obtained a stronger result about polynomial growth.

Theorem 8.3 (Gromov's effective polynomial growth theorem). For any positive integers $d$ and $k$, there exist positive integers $R, N$ and $q$ with the following property. If a group $G$ with a fixed system of generators satisfies the inequality $\gamma(n) \leq k n^{d}$ for $n=1,2, \ldots, R$ then $G$ contains a nilpotent subgroup $H$ of index at most $q$ and whose degree of nilpotence is at most $N$. 
In [Man07], A. Mann made the first step in the direction of getting a concrete effective bound on nilpotency class in Gromov's Theorem 8.1 by showing that if $G$ is a finitely generated group of polynomial growth of degree $d$, then $G$ contains a finite index nilpotent subgroup of nilpotence class at most $\sqrt{2 d}$. This is a corollary of the Bass-Guivarch formula (6.2). Mann also showed that $G$ contains a finite-by-nilpotent subgroup of index at most $g(d)$, the latter function being the maximal order of a finite subgroup of $G L_{d}(\mathbb{Z})$, which is known to be $2^{n} n$ ! in most cases (e.g. for $d=1,3,5$ and $d \geq 11$ ), [Man12, Theorem 9.8].

Another fact obtained by Gromov in [Gro81a] is the following: "If an infinitely generated group $G$ has no torsion and each finitely generated subgroup $G_{1}<G$ has polynomial growth of degree at most $d$, then $G$ contains a nilpotent subgroup of finite index". On the other hand, A. Mann showed in [Man07] that the property of an infinitely generated group $G$ being virtually nilpotent and of finite rank is equivalent to the property that there are some (fixed) constants $C$ and $d$ such that $\gamma_{H}^{A}(n) \leq C n^{d}, n=1,2, \ldots$ for every finitely generated subgroup $H<G$ with finite system of generators $A$.

Gromov in [Gro81a] raised the following question.

Gromov's Problem on Growth (I). What is the dependence of the numbers $R, N$ and $q$ on $d$ and $k$ ? In particular, does there exist an effective estimate of these numbers in terms of $d$ and $k$ ?

The second part of this question was answered by Y. Shalom and T. Tao in their "Quantitative Gromov Theorem" [ST10, Theorem 1.8] (see below for more on results of Shalom and Tao).

The theorem of Gromov was improved by van den Dries and Wilkie [vdDW84]. They showed that polynomial growth takes place under a weaker assumption: that the inequality $\gamma(n) \leq C n^{d}$ holds for infinite number of values of the argument $n$ (i.e. that the group has weakly polynomial growth in our terminology, see (3.6)). Van den Dries and Wilkie applied techniques of nonstandard analysis and generalized the notion of asymptotic cone using ultrafilters.

The original proof of Gromov (as well as its modification by van den Dries and Wilkie, see also the paper of Tits [Tit81]) is based on the use of the Gleason, Montgomery, Zippin, Yamabe structural theory of locally compact groups [MZ74]. For a long time there was a hope to find a proof of Gromov's theorem which would avoid the use of this remarkable (but technically very complicated) machinery which describes a relation between locally compact groups and Lie groups. This goal was achieved by B. Kleiner [Kle10] who proved Gromov's theorem using a completely different approach that involves harmonic functions. The core of Klener's arguments is in the new proof of (a slight modification of) the theorem of Colding-Minicozzi [CM97].

Theorem 8.4. (Kleiner 2010) Let $G$ be a group of weakly polynomial growth, and $l \in$ $(0, \infty)$. Then the space of harmonic functions on $\Gamma$ with polynomial growth at most $l$ is finite dimensional.

Recall that a function $f$ on a group $G$ is called $\mu$-harmonic if it satisfies $M f=f$, where $M$ is the Markov operator of the random walk on $G$ determined by the measure $\mu$. A function $f$ has at most polynomial growth of degree $l$ if for some positive constant $C$ the inequality $|f(g)| \leq C|g|^{l}$ holds, for all $g \in G$. 
Gromov's effective polynomial growth theorem was improved by Y. Shalom and T. Tao in different directions [ST10]. They showed that for some absolute (and explicit) constant $C$, the following holds for every finitely generated group $G$, and all $d>0$ : If there is some $R_{0}>\exp \left(\exp \left(C d^{C}\right)\right)$ for which $\gamma_{G}\left(R_{0}\right)<R_{0}^{d}$, then $G$ has a finite index subgroup which is nilpotent of nilpotency degree less than $C^{d}$. In addition, an effective upper bound on the index is provided in [ST10] if the word "nilpotent" is replaced by "polycyclic".

In [ST10], a pair $(G, A)$ (a group $G$ with a finite generating set $A$ ) is called an $\left(R_{0}, d\right)$ growth group if

$$
\gamma_{G}^{A}\left(R_{0}\right) \leq R_{0}^{d}
$$

Theorem 8.5 (Fully quantitative weak Gromov theorem). Let $d>0$ and $R_{0}>0$, and assume that $R_{0}>\exp \left(\exp \left(c d^{c}\right)\right)$ for some sufficiently large absolute constant $c$. Then every $\left(R_{0}, d\right)$-growth group has a normal subgroup of index at most $\exp \left(R_{0} \exp \left(\exp \left(d^{c}\right)\right)\right)$ which is polycyclic.

Corollary 8.6 (Slightly super-polynomial growth implies virtual nilpotency). Let $(G, A)$ be a finitely generated group such that $\gamma_{G}^{A}(n)<n^{c(\log \log n)^{c}}$ for some $n>1 / c$, where $c>0$ is a sufficiently small absolute constant. Then $G$ is virtually nilpotent.

This result shows that if the growth of a group is less than that of $n^{(\log \log n)^{c}}$, then it is polynomial.

Gromov's original proof implies the existence of a function $v(n)$ growing faster than any polynomial and such that if $\gamma_{G}(n) \prec v(n)$, then the growth of $G$ is polynomial. Therefore there is a gap in the scale of growth degrees of finitely generated groups. In fact, as there are groups with incomparable growth functions, it may happen that there can be many gaps between polynomial and intermediate growth.

The function $n^{(\log \log n)^{c}}$ is the first concrete example of the superpolynomial bound separating the polynomial growth case from the intermediate one. Finding the border(s) between polynomial and intermediate growth is one of the main open problems about the growth of groups. We call this the Gap Problem and there is the associated Gap Conjecture which we discuss in more detail in section 11.

One more result in the direction of study of polynomial growth is the characterization of finitely generated cancellative semigroups of polynomial growth given in [Gri88]. Recall that a semigroup $S$ is cancellative if the left and right cancellative laws hold: $\forall a, b, c \in S, a b=$ $a c \Rightarrow b=c, b a=c a \Rightarrow b=c$. Cancelation is a necessary condition for embedding of a semigroup into a group. In 1957, A.I. Malcev introduced the notion of a nilpotent semigroup [Mal53]. A semigroup is nilpotent if for some $n \geq 1$, it satisfies the identity $X_{n}=Y_{n}$, where $X_{n}, Y_{n}, n=0,1, \ldots$ are words over the alphabet $x, y, z_{1}, z_{2}, \ldots, z_{n}, \ldots$ defined inductively: $X_{0}=x, Y_{0}=y$ and

$$
X_{n+1}=X_{n} z_{n} Y_{n}, \quad Y_{n+1}=Y_{n} z_{n} X_{n}
$$

A semigroup is of nilpotency degree $n$ if it satisfies the identity $X_{n}=Y_{n}$ but not the identity $X_{n-1}=Y_{n-1}$. Malcev proved that a group $G$ is nilpotent of degree $n$ if and only if it is nilpotent of degree $n$ as a semigroup.

We say that a subsemigroup $L \leq S$ has a finite (left) index in $S$ if there is a finite subset $E=\left\{e_{1}, \ldots, e_{k}\right\} \subset S$ such that

$$
S=\bigcup_{i=1}^{k} e_{i} L
$$


Theorem 8.7 ([Gri88]). Let $S$ be a cancellative semigroup of polynomial growth of degree d i.e.

$$
d=\varlimsup_{n \rightarrow \infty} \frac{\log \gamma_{S}(n)}{\log n}<\infty
$$

Then

(i) $S$ has a group $G=S^{-1} S$ of left quotients which also has polynomial growth of degree $d$ and therefore is virtually nilpotent. In particular, $d$ is a nonnegative integer.

(ii) The semigroup $S$ is virtually nilpotent in Malcev sense. More precisely, $G$ contains a nilpotent subgroup $H$ of finite index such that $S_{1}=H \cap S$ is a nilpotent semigroup of the same degree of nilpotency as $H$, and $S_{1}$ is of finite (left or right) index in $S$.

The proof of this theorem relies on the fact that a cancellative semigroup $S$ of subexponential growth satisfies the Ore condition and hence has a group of left quotients $G$ (this also holds for cancellative right amenable semigroups). The next step consists in getting of an upper bound of polynomial type for the growth of $G$, and an application of Gromov's theorem finishes the argument.

Observe that much less is known about growth of nilpotent cancellative semigroups than nilpotent groups. For instance, it is unknown if there is a constant $c>0$ such that

$$
c n^{d} \leq \gamma_{S}(n), n=1,2, \ldots
$$

in the case when $S$ is a cancellative semigroup of polynomial growth of degree $d$. In the case of a semigroup without cancellation, the growth can be equivalent to the growth of an arbitrary function $\gamma(n)$ satisfying reasonable restrictions, as was shown by V. Trofimov in [Tro80]. Also a semigroup of polynomial growth need not be virtually nilpotent in Malcev sense.

We conclude this section with the following questions.

Problem 2. Let $S$ be a cancellative semigroup of polynomial growth of degree $d$. Is it true that the limit

$$
d=\lim _{n \rightarrow \infty} \frac{\gamma_{S}(n)}{n^{d}}
$$

exists?

Problem 3. Does there exist a finitely generated cancellative semigroup $S$ of subexponential growth such that the group $G=S^{-1} S$ of left quotients has exponential growth?

A positive answer to the previous question would provide an example of a superamenable group of exponential growth and an answer to the J. Rosenblatt's question from [Ros74]. Also

observe that in the case $\gamma_{S}(n) \prec e^{\sqrt{n}}$, the group of quotients $G=S^{-1} S$ has subexponential growth which justifies the following question.

\section{Problem 4.}

(a) Does there exist a finitely generated cancellative semigroup $S$ of superpolynomial growth which is strictly less that the growth of $e^{\sqrt{n}}$ ?

(b) Does there exist a finitely generated cancellative semigroup $S$ of growth equivalent to the growth of $e^{\sqrt{n}}$ ? 
This problem is related to problem 10 and the Gap Conjecture discussed in section 10.

\section{INTERMEDIATE GROWTH: THE CONSTRUCTION}

The first part of Milnor's problem was solved by the author in 1984 .

Theorem 9.1. ([Gri84b])

- There are finitely generated groups of intermediate growth.

- The partially ordered set $\mathcal{W}$ of growth degrees of 2-generated groups contains a chain of the cardinality of the continuum, and contains an antichain of the cardinality of the continuum.

- The previous statement holds for the class of 2-generated p-groups for any prime p.

Corollary 9.2. There are uncountably many 2-generated groups, up to quasi-isometry.

The main example of a group of intermediate growth, which will be denoted through the whole paper by $\mathcal{G}$, is the group defined by the next figure:

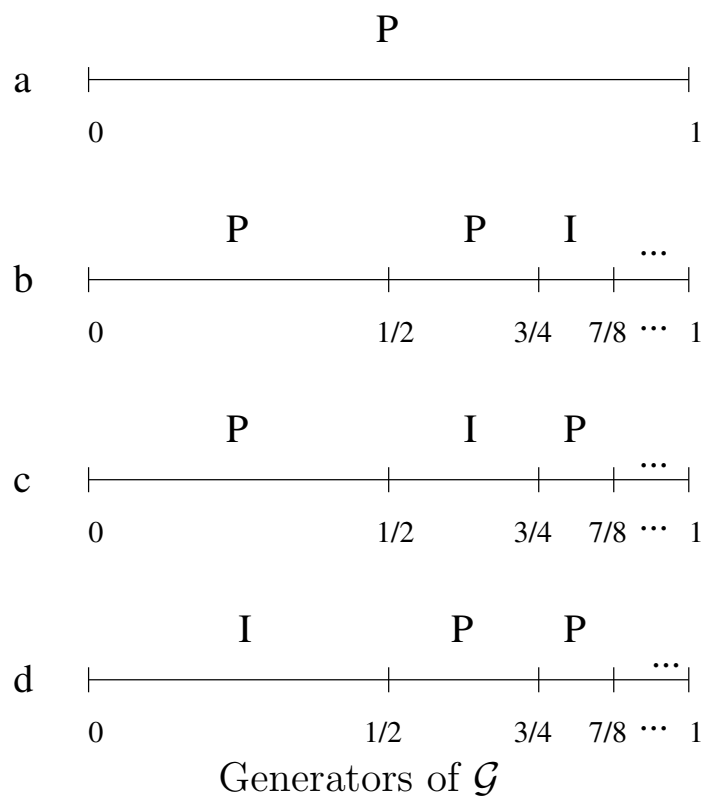

This group was constructed in 1980 in the author's note [Gri80b] as a simple example of a finitely generated infinite torsion group. Recall that the question on the existence of such groups was the subject of the General Burnside Problem raised by Burnside in 1904. The problem was solved by E. Golod in 1964 on the basis of the GolodShafarevich Theorem [GŠ64, Gol64]. The bounded version of the Burnside problem (when orders of all elements are assumed to be uniformly bounded) was solved by S. Novikov and S. Adian [NA68a, NA68c, NA68d, Adi79]). A third version of the Burnside Problem, known as the Restricted Burnside Problem, was solved by E. Zelmanov [Zel91a, Zel90, Zel91b].

The note [Gri80b] contains two examples of Burnside groups, and the second group $\mathcal{B}$ presented there was, in fact, historically constructed before group $\mathcal{G}$. Therefore, it can be considered as the first example of a nonelementary self-similar group (self-similar groups are discussed in the next section). The group $\mathcal{B}$ shares with $\mathcal{G}$ the property of being an infinite torsion group, but it is unknown whether it has intermediate growth. Also it is unknown 
if the Gupta-Sidki $p$-groups [GS83], whose construction and properties are similar to $\mathcal{B}$ in many aspects, have intermediate growth.

The group $\mathcal{G}$ acts on the unit interval $[0,1]$ with the dyadic rational points removed, i.e. on the set $\Delta=[0,1] \backslash\left\{m / 2^{n}, 0 \leq m \leq 2^{n}, n=1,2, \ldots\right\}$ (if needed, the action of $\mathcal{G}$ can be extended to the entire interval $[0,1))$. The generator $a$ is a permutation of the two halves of the interval, and $b, c, d$ are also interval exchange type transformations (but involve a partition of $\Delta$ into infinitely many subintervals). They preserve the partition of the interval indicated in the figure, and their action is described by the labeling of the atoms of partition by symbols $\{I, P\}$. The letter $I$ written over an interval means the action is the identity transformation there, and the letter $P$ indicates the two halves of the interval are permuted as indicated in the figure below.

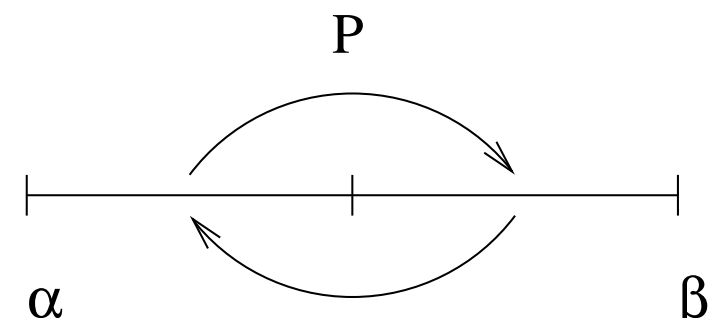

Now we are going to describe a general construction of uncountably many groups of intermediate growth. These groups are of the form

$$
\mathcal{G}_{\omega}=\left\langle a, b_{\omega}, c_{\omega}, d_{\omega}\right\rangle
$$

where the generator $a$ is defined as before, and $b_{\omega}, c_{\omega}, d_{\omega}$ are transformations of the set $\Delta$ defined using an oracle $\omega \in \Omega=\{0,1,2\}^{\mathbb{N}}$ in the same manner as $b, c, d$. To describe the construction of groups $\mathcal{G}_{\omega}$ completely, let us start with the space $\Omega=\{0,1,2\}^{\mathbb{N}}$ of infinite sequences over alphabet $\{0,1,2\}$. The bijection

$$
\begin{gathered}
0 \longleftrightarrow\left(\begin{array}{c}
P \\
P \\
I
\end{array}\right) \\
1 \longleftrightarrow\left(\begin{array}{c}
P \\
I \\
P
\end{array}\right) \\
2 \longleftrightarrow\left(\begin{array}{c}
I \\
P \\
P
\end{array}\right)
\end{gathered}
$$

between symbols of the alphabet $\{0,1,2\}$ and corresponding columns is used in the construction. Namely given a sequence $\omega=\omega_{1} \omega_{2} \ldots \in \Omega$ replace each $\omega_{i}, i=1,2, \ldots$, by the corresponding column to get a vector

$$
\left(\begin{array}{c}
U_{\omega} \\
V_{\omega} \\
W_{\omega}
\end{array}\right)
$$


consisting of three infinite words $U_{\omega}, V_{\omega}, W_{\omega}$ over the alphabet $\{I, P\}$. For instance, the triple of words

$$
\begin{aligned}
U_{\xi} & =P P I P P I \ldots \\
V_{\xi} & =P I P \text { PIP } \ldots \\
W_{\xi} & =I P P I P P \ldots
\end{aligned}
$$

corresponds to the sequence $\xi=012012 \ldots$, while the triple

$$
\begin{aligned}
U_{\eta} & =P P P P P P \ldots \\
V_{\eta} & =P I P I P I \ldots \\
W_{\eta} & =I P I P I P \ldots
\end{aligned}
$$

corresponds to the sequence $\eta=0101 \ldots$

Using the words $U_{\omega}, V_{\omega}, W_{\omega}$, define transformations $b_{\omega}, c_{\omega}, d_{\omega}$ of the set $\Delta$ as in the case of the sequence $\xi$; the sequence $\xi$ corresponds to the group $\mathcal{G}$. Then $\mathcal{G}_{\omega}=\left\langle a, b_{\omega}, c_{\omega}, d_{\omega}\right\rangle$. The most interesting groups from this family correspond to sequences $\xi$ and $\eta$, which are respectively the groups $\mathcal{G}$ and $\mathcal{E}=\mathcal{G}_{(01)^{\infty}}$ studied by A. Erschler [Ers04a].

The generators of $\mathcal{G}_{\omega}$ satisfy the relations

$$
\begin{aligned}
& a^{2}=b_{\omega}^{2}=c_{\omega}^{2}=d_{\omega}^{2}=1 \\
& b_{\omega} c_{\omega}=c_{\omega} b_{\omega}=d_{\omega} \\
& b_{\omega} d_{\omega}=d_{\omega} b_{\omega}=c_{\omega} \\
& c_{\omega} d_{\omega}=d_{\omega} c_{\omega}=b_{\omega}
\end{aligned}
$$

(this is only a partial list of the relations), from which it follows that the groups $\mathcal{G}_{\omega}$ are 3-generated (and even 2-generated in some degenerate cases, for example, the case of the sequence $00 \ldots 0 \ldots)$. Nevertheless, we prefer to consider them as 4-generated groups, with the system $\left\{a, b_{\omega}, c_{\omega}, d_{\omega}\right\}$ viewed as a canonical system of generators.

While the groups $\mathcal{G}_{\omega}$ are 3 -generated, a simple trick used in [Gri84b] produces a family $\mathcal{H}_{\omega}, \omega \in \Omega$ of 2 -generated groups with the property that for $\omega \in \Omega_{1}$ (the set $\Omega_{1}$ is defined below), the growth of $\mathcal{H}_{\omega}$ is the same as growth of $\mathcal{G}_{\omega}$ raised to the fourth power.

Alternatively, the groups $\mathcal{G}_{\omega}$ can be defined as groups acting by automorphism on a binary rooted tree $T$ depicted in the following figure. 


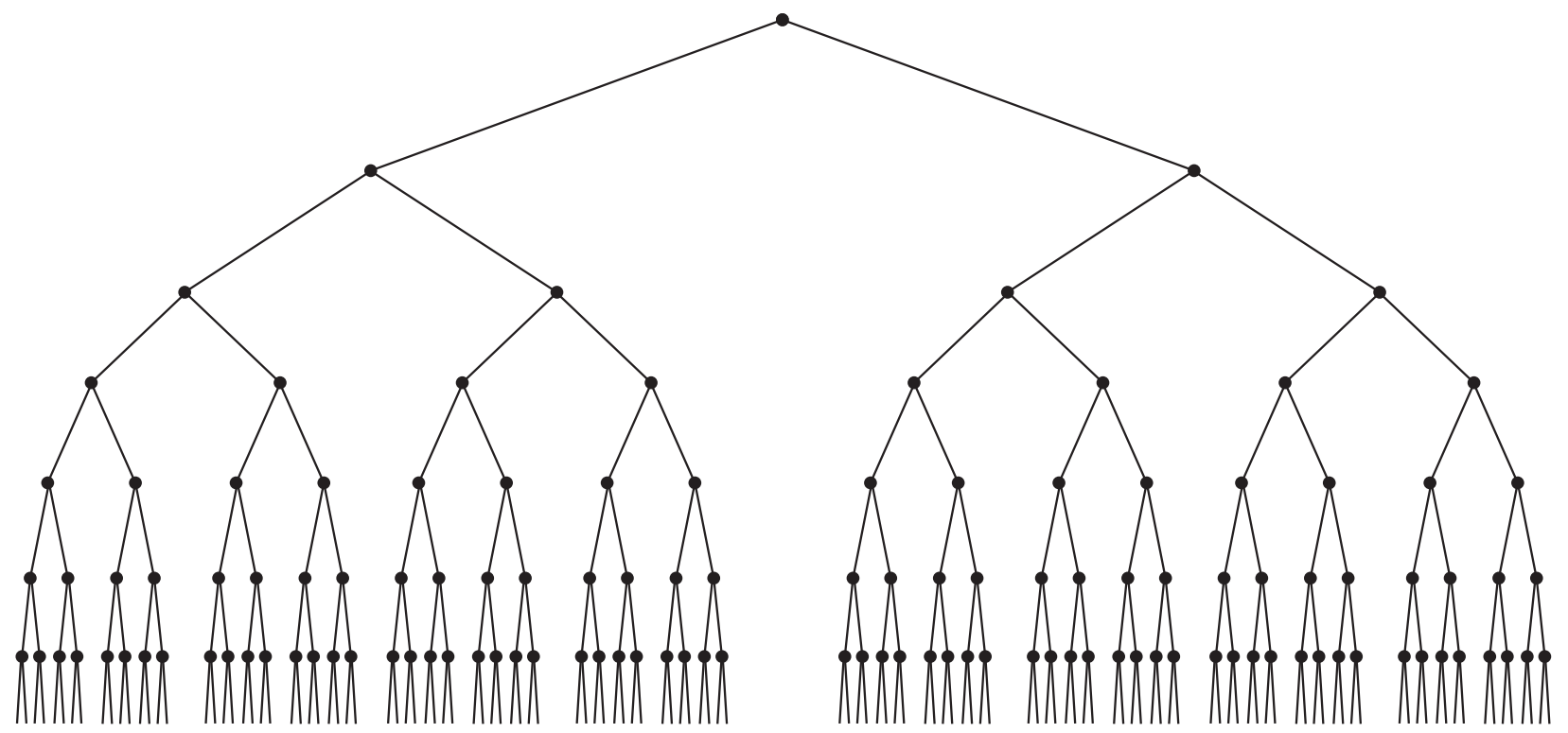

For instance, let us explain how generators of $\mathcal{G}$ act on $T$. The generator $a$ acts by permuting the two rooted subtrees $T_{0}$ and $T_{1}$ which have roots at the first level. The generator $b$ acts on the left subtree $T_{0}$ as $a$ acts on $T$ (here we use the self-similarity property of a binary tree), and acts on the right subtree $T_{1}$ as generator $c$ (whose action on the whole tree is shifted to the right subtree). Similarly, $c$ acts on $T_{0}$ as $a$ on $T$ and on $T_{1}$ as $d$ acts on $T$. And finally, $d$ acts on $T_{0}$ as the identity automorphism and on $T_{1}$ as $b$ on $T$. This gives us a recursive definition of automorphisms $b, c, d$ as in the figure below, where dotted arrows show the action of a generator on the corresponding vertex.
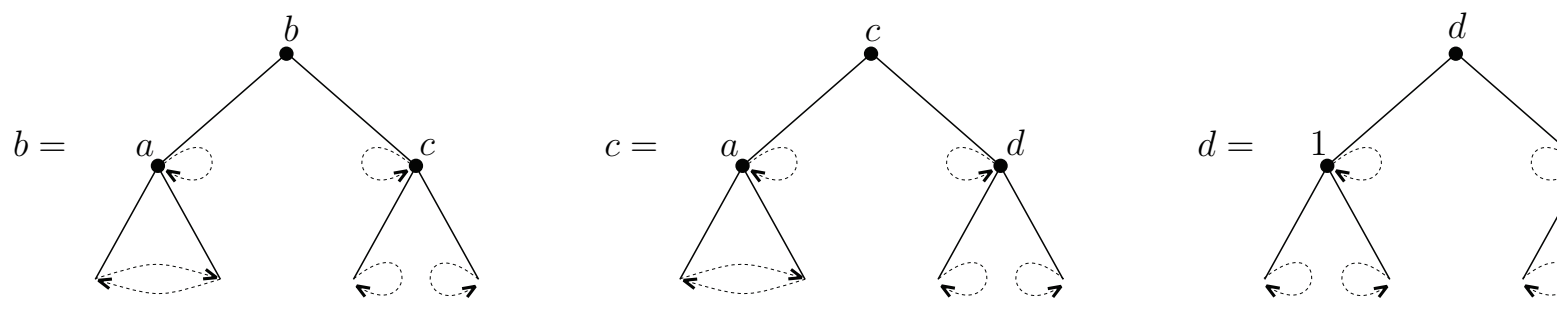

Making the identification of vertices of $T$ with finite binary sequences, identifying the boundary $\partial T$ of the tree with the set of infinite sequences from $\{0,1\}^{\mathbb{N}}$, and representing dyadic irrational points by the corresponding binary expansions, we obtain an isomorphism of the dynamical system $(\mathcal{G},[0,1], m)$ (where $m$ is Lebesgue measure) with the system $(\mathcal{G}, \partial T, \nu)$, where $\nu$ is a uniform measure on the boundary $\partial T$ (i.e. a $\left\{\frac{1}{2}, \frac{1}{2}\right\}$ Bernoulli measure if the boundary is identified with the space of binary sequences). A similar description of the action on a binary tree can be given for all groups $\mathcal{G}_{\omega}$, although self-similarity should be used in a more general sense.

There are many other ways to define the groups $\mathcal{G}_{\omega}$. For instance, a very nice approach was suggested by Z. Sunic which allows one to define the groups $\mathcal{G}$ and $\mathcal{E}$ by use of irreducible (in the ring $\mathbb{F}_{2}[x]$ ) polynomials $x^{2}+x+1$ and $x^{2}+1$ respectively. This approach allows one to associate to any irreducible polynomial a self-similar group [BGŠ03].

One more definition of the group $\mathcal{G}$ can be given by the following presentation found by I. Lysenok [Lys85]: 


$$
\mathcal{G}=\left\langle a, b, c, d \mid a^{2}, b^{2}, c^{2}, d^{2}, b c d, \sigma^{k}\left((a d)^{4}\right), \sigma^{k}\left((a d a c a c)^{4}\right), k \geq 0\right\rangle,
$$

where

$$
\sigma:\left\{\begin{array}{l}
a \rightarrow a c a, \\
b \rightarrow d, \\
c \rightarrow b, \\
d \rightarrow c .
\end{array}\right.
$$

is a substitution. A similar presentation (called an $L$-presentation) can be found for $\mathcal{E}$ and many other self-similar groups.

Theorem 9.3. ([Gri80b, Gri84b])

1) $\mathcal{G}$ is a residually finite torsion D-group, which is not finitely presentable.

2) $\mathcal{G}$ is of intermediate growth with bounds

$$
e^{n^{1 / 2}} \preceq \gamma_{\mathcal{G}}(n) \preceq e^{n^{\beta}},
$$

where $\beta=\log _{32} 31<1$.

3) (A. Erschler [Ers04a]) For any $\epsilon>0$ the following upper and lower bounds hold:

$$
\exp \frac{n}{\log ^{2+\epsilon} n} \prec \gamma_{\mathcal{E}}(n) \prec \exp \frac{n}{\log ^{1-\epsilon} n} .
$$

4) ([Gri85a]) There is a torsion free extension $\hat{\mathcal{G}}$ of abelian group of infinite rank by $\mathcal{G}$ which has intermediate growth.

The first part of this theorem shows that there are examples of groups of Burnside type already in the class of groups of intermediate growth. The last part shows that there are torsion free groups of intermediate growth.

The question of finding the precise (in Milnor-Schwarz sense) growth degree of any group of intermediate growth was open until recently, when in their excellent paper [BA10], Bartholdi and Erschler constructed groups with growth of the type $\exp n^{\alpha}$ for infinitely many $\alpha \in$ $[0.7674,1)$ (more on their result in section 13). Moreover, they were able to find the growth degree of the torsion free group $\hat{\mathcal{G}}$ by showing that

$$
\gamma_{\hat{\mathcal{g}}}(n) \sim n^{\log (n) n_{0}^{\alpha}},
$$

where $\alpha_{0} \approx 0.7674$, is defined below (9.2). In addition to the properties of $\hat{\mathcal{G}}$ listed above, we have the following fact, which was established in the paper of A. Machi and author [GM93].

Theorem 9.4. The group $\hat{\mathcal{G}}$ is left orderable and hence acts by orientation preserving homeomorphisms of the line $\mathbb{R}$ (or, equivalently, of the interval $(0,1)$ ).

It is unknown if there are orderable groups (i.e. groups possessing a two-sided invariant order) of intermediate growth.

Problem 5. Does there exist a finitely generated, orderable group of intermediate growth?

A possible approach to this problem could be the following. By a theorem of M.I. Zaiceva [KK74], if a factor group $F / A$ of a free group $F$ has an infrainvariant system of subgroups (for the definition, see [KK74]) with torsion free abelian quotients, then the group $G / A$ is left orderable. Moreover, by D.M. Smirnov's theorem [Smi64, KK74] the group 
$F /[A, A]$ is totally orderable in this case. It is known that the group $\hat{\mathcal{G}}$ has an infrainvariant system of subgroups with torsion free abelian quotients. Representing the group $\hat{\mathcal{G}}$ in the form $F / A$ we obtain an interesting totally orderable group $F /[A, A]$. The question is whether it is of intermediate growth.

The last theorem shows that $\mathcal{G}$ embeds into the group $\mathrm{Homeo}^{+}([0,1])$ of homeomorphisms of the interval. The notion of growth is useful for study of codimension one foliations, and group growth as well as growth of Schreier graphs play important role. The question concerning the smoothness of the action of a group on a manifold arises in many situations. Many topics related to the subject of actions on the interval and on the circle can be found in [Ghy01, Nav11, Bek04, Bek08]. The following result of A. Navas [Nav08] gives important information about what one can expect in the one-dimensional case.

Theorem 9.5. The group $\mathcal{G}$ embeds into Diff ${ }^{1}([0,1])$. However, for every $\epsilon>0$, every subgroup of Diff ${ }^{1+\epsilon}([0,1])$ without a free subsemigroup on two generators is virtually nilpotent.

Until 2004, all known examples of groups of intermediate growth were residually finite groups. The question about existence of non residually finite groups of intermediate growth was open for a while. In [Ers04b], Erschler constructed an uncountable family of non residually finite groups of intermediate growth. These groups are extensions of an elementary 2-group of infinite rank by $\mathcal{G}$.

Let $\Omega_{0} \subset \Omega$ be the subset consisting of sequences in which all three symbols $0,1,2$ occur infinitely often, let $\Omega_{1} \subset \Omega$ be the subset consisting of sequences in which at least two of the symbols $0,1,2$ occur infinitely often, and let $\Theta \subset \Omega$ be the subset consisting of sequences $\omega$ with the property that there is a natural number $C=C(\omega)$ such that for every $n$, each of symbols $0,1,2$ appears among any $C$ consecutive symbols $\omega_{n} \omega_{n+1} \ldots \omega_{n+C-1}$. Observe that the inclusions $\Theta \subset \Omega_{0} \subset \Omega_{1}$ hold.

Recall that a group is called just-infinite if it is infinite but every proper quotient is finite. We postpone the definition of branch groups (as well as the notions of self-similar group and contracting group) until section 9.

Theorem 9.6. ([Gri84b])

- The word problem for the group $\mathcal{G}_{\omega}$ is solvable if and only if the sequence $\omega$ is recursive.

- For each $\omega \in \Omega_{0}$ the group $\mathcal{G}_{\omega}$ is a just-infinite torsion 2-group.

- For each $\omega \in \Omega_{1}$ the group $\mathcal{G}_{\omega}$ is infinitely presented and branch.

- For each $\omega \in \Omega_{1}$ the group $\mathcal{G}_{\omega}$ has intermediate growth and the lower bound

$$
e^{n^{1 / 2}} \preceq \gamma_{G_{\omega}}(n)
$$

holds.

- For each $\theta \in \Theta$ there is a $\beta=\beta(\theta)<1$ such that

$$
\gamma_{G_{\omega}}(n) \preceq e^{n^{\beta}}
$$

holds.

The first statement of this theorem shows that there are many groups of intermediate growth for which the word problem is decidable, and therefore there are recursively presented groups of intermediate growth. At the same time there are recursively presented residually finite groups of intermediate growth for which the word problem is unsolvable, as is shown in [Gri85b]. 
Formally speaking [Gri84b] does not contain the proof of the last part of the last theorem. But it can be easily obtained following the same line as the proof of theorem 3.2 from [Gri84b].

The lower bound by the function $e^{\sqrt{n}}$ for the growth of $\mathcal{G}$ was improved by L. Barthodi [Bar01] and Y. Leonov [Leo01], who showed that $\exp n^{0.5157} \preceq \gamma_{\mathcal{G}}(n)$.

The upper bound for $\mathcal{G}$ given by theorem 9.3 was improved by L. Bartholdi [Bar98] by showing that

$$
\gamma_{\mathcal{G}}(n) \preceq e^{n^{\alpha_{0}}}
$$

with $\alpha_{0}=\log 2 / \log (2 / \rho) \approx 0.7674$, where $\rho$ is the real root of the polynomial $x^{3}+x^{2}+x-2$. The method used consists of consideration of a more general type of length function, arising from prescribing positive weights to generators, and counting the length using the weighted contribution of each generator. The method relies on the idea of giving the optimal weights to generators that lead to the best upper bound. It happened to be also useful for the construction of groups of intermediate growth with explicitly computed growth, as recently was demonstrated by Bartholdi and Erschler in [BA10] (more on this in section 13).

Another technical tool was explored by R. Muchnik and I. Pak [MP01a] to get an upper bound on growth for the whole family of groups $\left\{\mathcal{G}_{\omega}\right\}$. Surprisingly, in the case of $\mathcal{G}$ their approach give the same upper bound as (9.2), so the question of improving it is quite intriguing (see problem 7).

Unfortunately, even after three decades of study of the group $\mathcal{G}$ and other groups $\mathcal{G}_{\omega}$, we still do not know the precise growth rate of any group of intermediate growth from the family $\left\{\mathcal{G}_{\omega}\right\}$.

\section{Problem 6.}

(1) Does there exist $\alpha$ such that $\gamma_{\mathcal{G}}(n) \sim e^{n^{\alpha}}$ ?

(2) If the answer to the previous question is "yes", what is the value of $\alpha$ ?

The question of whether the upper bound obtained in [BA10, MP01a] is optimal (i.e. it coincides with the growth rate of the group $\mathcal{G}$ ) is very intriguing and we formulate it as the next problem. The point is that all known groups whose growth is explicitly computed up to equivalence by $\sim$ (i.e. groups considered in [BA10, BE11]) have growth not smaller than $e^{n^{\alpha_{0}}}$. And it looks that if the growth of $\mathcal{G}$ is less than $e^{n^{\alpha_{1}}}$ with $\alpha_{1}<\alpha_{0}$ then, using the results of the cited papers and of the paper by Kassabov and Pak [KP11], one can extend the range of possible growth rates from the "interval" $\left[e^{n^{\alpha_{0}}}, e^{n}\right]$ to the "interval" $\left[e^{n^{\alpha_{1}}}, e^{n}\right]$. But so far $e^{n^{\alpha_{0}}}$ is a kind of a "mountain" which "closes the sky" for people working in the area of group growth.

In view of recent results from [BA10, Bri11], even obtaining a weaker result that would answer the next question seems to be interesting in its own right.

\section{Problem 7.}

(1) Is the upper bound (9.2) the best possible for $\mathcal{G}$ ?

(2) Does there exist a group of intermediate growth whose growth is less than $e^{n^{\alpha_{1}}}$, where $\alpha_{1}<\alpha_{0}$ and $\alpha_{0}=\log 2 / \log (2 / \rho)$ is the constant defined above?

In section 11, we will give a brief account of methods that can be used to obtain upper and lower bounds for intermediate growth. 
The groups $\mathcal{G}$ and $\mathcal{E}$ belong to the class of self-similar groups, that is, groups generated by automata of Mealy type, which are discussed a bit in the next section. An important quantative characteristic of such groups is the pair $(m, n)$ which is a rough indication of the complexity of the group, where $m$ is the cardinality of the alphabet and $n$ is the cardinality of the set of the states of automaton. From this point of view, $\mathcal{G}_{\xi}$ and $\mathcal{G}_{\eta}$ are $(2,5)$-groups (the Moore diagrams of corresponding automata are presented in the next section). And there is even a group of intermediate growth of complexity $(2,4)$ (namely the iterated monodromy group $I M G\left(z^{2}+i\right)$ in the sence of Nekrashevych [Nek05] of quadratic polynomial $\left.z^{2}+i\right)$ as was showed by K. Bux and R. Peres [BP06].

\section{Problem 8.}

(i) Find the growth degree of each of the groups $\mathcal{G}_{\xi}, \mathcal{G}_{\eta}$, and $\operatorname{IMG}\left(z^{2}+i\right)$.

(ii) Are there groups of intermediate growth of complexity $(2,3)$ ?

(iii) Determine all automata of complexity $(2,3),(2,4)$ and $(2.5)$ which generate groups of intermediate growth.

(iv) Determine all possible types of growth of self-similar groups generated by finite automata.

It is a kind of a miracle that an automaton with a small number of states can generate a group with very complicated algebraic structure and asymptotic behavior. Therefore, it is not surprising that some of the automata groups studied prior to 1983 (when the the first examples of groups of intermediate growth were found) are also of intermediate growth. Specifically, the 2-group of Aleshin [Ale72] (generated by two automata with 3 and 7 states) and the $p$-groups of V. Sushchanskii (generated by automata with a number of states growing as a quadratic function of $p$ [Sus79]) were shown to have intermediate growth by the author in [Gri84b, page 280] and [Gri85a, page 197], respectively (see also [BS07] where the Sushchanskii group is treated in detail). It is worth mentioning that the papers of Aleshin and Sushchanskii deal exclusively with the question of construction of finitely generated infinite torsion groups (contributing to the General Burnside Problem) and Milnor's problem is not considered at these articles at all.

As all the groups of intermediate growth from theorem 9.6 have only finite quotients (and consequently, at most a countable set of quotients), in 1983 it was reasonable to ask if there are groups of intermediate growth with uncountably many homomorphic images, one of the properties that a finitely generated virtually nilpotent group does not have. This was affirmatively answered in [Gri84a]. The next theorem gives a hint to the main result of [Gri84a] and its proof.

Let $\Lambda \subset \Omega$ be the subset consisting of sequences that are products of blocks $012,120,201$, and let $\mathcal{G}_{\lambda}$ be presented as the quotient $F_{4} / \mathcal{N}_{\lambda}$ of the free group $F_{4}$ of rank 4 by a normal subgroup $\mathcal{N}_{\lambda}$. Call the group $\mathcal{U}_{\Lambda}=F_{4} / \bigcap_{\lambda \in \Lambda} \mathcal{N}_{\lambda} \Lambda$-universal.

Theorem 9.7. The $\Lambda$-universal group $\mathcal{U}_{\Lambda}$ has intermediate growth and has uncountably many quotients which are pairwise non-isomorphic.

The first part of the theorem follows from [Gri84b, theorem 3.2].

As each of the groups $\mathcal{G}_{\lambda}$ is a homomorphic image of $\mathcal{U}_{\Lambda}$, the second part of the theorem is obvious modulo the fact that the classes of isomorphisms of groups from the family $\mathcal{G}_{\omega}$, $\omega \in \Omega$ are at most countable. It was shown by Nekrashevych in [Nek07] that $\mathcal{G}_{\omega} \simeq \mathcal{G}_{\zeta}$ if and only if the sequence $\zeta$ can be obtained from $\omega$ by the diagonal action at all coordinates 
of an element from the symmetric group $\operatorname{Sym}(3)$ acting on the set $\{0,1,2\}$. (In fact, it is enough to quote theorem 5.1 from [Gri84b], which states that for each $\omega$ there are at most countably many groups $\mathcal{G}_{\eta}$ isomorphic to $\mathcal{G}_{\omega}$ ).

In contrast with the $\Lambda$-universal group $\mathcal{U}_{\Lambda}$, the growth of the $\Omega$-universal group $\mathcal{U}_{\Omega}=$ $F_{4} / \bigcap_{\omega \in \Omega} \mathcal{N}_{\omega}$ is exponential [Muc05]. It is known that $\mathcal{U}_{\Omega}$ does not contain a free subgroup on two generators, is self-similar (of complexity $(6,5)$ [Gri05]), weakly branch and contracting. However, the following question is still open (unfortunately the article [Muc05], which contains the claim about amenability of $\mathcal{U}_{\Omega}$, has a mistake).

Problem 9. Is $\mathcal{U}_{\Omega}$ amenable or not?

In [Gri84b] the author proved that for any monotone function $\rho(n)$ growing slower than exponential functions, there is a group with growth not slower than $\rho(n)$ (so either $\rho(n) \preceq$ $\gamma_{G}(n)$ or $\rho(n)$ and $\gamma_{G}(n)$ are incomparable with respect to the preoder $\left.\preceq\right)$. This result was improved by Erschler .

Theorem 9.8. (Erschler [Ėrs05b]) For any increasing function $\rho(n)$ growing slower than an exponential function, there is a finitely generated group $G$ of intermediate growth with $\rho(n) \preceq \gamma_{G}(n)$.

The last result shows that there is no upper bound for intermediate growth, in contrast with the lower bound given by the Shalom-Tao function $n^{c(\log \log n)^{c}}$ ( $c$ a constant), as discussed in section 8 .

\section{The Gap Conjecture}

The history of the Gap Conjecture is as follows. While reading Gromov's paper on polynomial growth in 1982 (soon after its publication), the author realized that the effective version of Gromov's polynomial growth theorem (Theorem 8.1) implies the existence of a function $v(n)$ growing faster than any polynomial such that, if $\gamma_{G}(n) \prec v(n)$, then the growth of $G$ is polynomial. Indeed, taking a sequence $\left\{k_{i}, d_{i}\right\}_{i=1}^{\infty}$ with $k_{i} \rightarrow \infty$ and $d_{i} \rightarrow \infty$ as $i \rightarrow \infty$ along with the corresponding sequence $\left\{R_{i}\right\}_{i=1}^{\infty}$ (whose existence follows from Theorem 8.3), one can build a function $v(n)$ which coincides with the polynomial $k_{i} n^{d_{i}}$ on the interval $\left[R_{i-1}+1, R_{i}\right]$. The constructed function $v(n)$ grows faster than any polynomial and separates polynomial growth from intermediate growth. In fact, as was already mentioned in section 8 and at the end of previous section, the function $n^{c(\log \log n)^{c}}(c$ a constant) separates polynomial growth from exponential [ST10]. As, according to Theorem 9.1, the set of growth degrees is not linearly ordered, it may happen that there is more than one "gap" between polynomial growth and intermediate growth. But in any case, it would be nice to obtain the best possible estimate of the asymptotics of a function which "uniformly" separates the polynomial and intermediate growth.

Approximately at the same time, while reading Gromov's paper (thus around 1982), the author was establishing his results on groups of intermediate growth discussed in the previous section and in 1983-1985 published [Gri83, Gri84b, Gri85a]. The lower bound of the type $e^{\sqrt{n}}$ for all groups $\mathcal{G}_{\omega}$ of intermediate growth established in those papers and in his Habilitation [Gri85b] allowed to author to guess that the equivalence class of the function $e^{\sqrt{n}}$ could be a good candidate for a "border" between polynomial and exponential growth. This guess became stronger in 1988 when the author obtained the results published in [Gri89a] (see Theorem 10.1). In the ICM Kyoto paper [Gri91], the author raised a question of whether the 
function $e^{\sqrt{n}}$ gives a universal lower bound for all groups of intermediate growth. Moreover, at approximately the same time, he conjectured that indeed this is the case, and stated this later at numerous talks.

Conjecture 2. (Gap Conjecture) If the growth function $\gamma_{G}(n)$ of a finitely generated group $G$ is strictly bounded from above by $e^{\sqrt{n}}$ (i.e. if $\gamma_{G}(n) \prec e^{\sqrt{n}}$ ), then the growth of $G$ is polynomial.

We are also interested to know whether there is a group, or more generally a cancellative semigroup, with growth equivalent to $e^{\sqrt{n}}$.

In this section we formulate several results in the direction of confirmation of the Gap Conjecture, and suggest slightly different versions of it. Later in section 12 we will formulate analogous conjectures about some other asymptotic characteristics of groups.

The next few results, together with the results about lower bounds on growth discussed in the next section, are the main source of support of the Gap Conjecture. Recall that a group $G$ is said to be a residually finite- $p$ group (sometimes also called residually finite $p$-group) if it is approximated by finite $p$-groups, i.e. for any $g \in G$ there is a finite $p$-group $H$ and a homomorphism $\phi: G \rightarrow H$ with $\phi(g) \neq 1$. This class is, of course, smaller than the class of residually finite groups, but it is pretty large. For instance, it contains Golod-Shafarevich groups, the $p$-groups $\mathcal{G}_{\omega}$ from [Gri84b, Gri85a], and many other groups.

Theorem 10.1. ([Gri89a]) Let $G$ be a finitely generated residually finite-p group. If $\gamma_{G}(n) \prec$ $e^{\sqrt{n}}$ then $G$ has polynomial growth.

As was established by the author in a discussion with A. Lubotzky and A. Mann during the conference on profinite groups in Oberwolfach in 1990, the same arguments as given in [Gri84b] combined with Lemma 1.7 from [LM91] allow one to prove a stronger version of the above theorem (see also the remark after Theorem 1.8 in [LM91], but be aware that capital $O$ has to be replaced by small $o$ ).

Theorem 10.2. Let $G$ be a residually nilpotent finitely generated group. If $\gamma_{G}(n) \prec e^{\sqrt{n}}$ then $G$ has polynomial growth.

The main goal of the paper [LM91], followed by the article [LMS93], was to give a complete description of finitely generated groups with polynomial subgroup growth (the growth of the function which counts the number of subgroups of given finite index). The remarkable result achieved in [LMS93] shows that such groups are precisely the solvable groups of finite rank.

As was already mentioned in the introduction, the original proof of Gromov's polynomial growth theorem is based on the use of the solution of Hilbert's 5th problem by Montgomery and Zippin, concerning the isometric actions of locally compact groups and their relation to Lie groups. Surprisingly, in the proofs of the results stated in Theorems 10.1 and 10.2, as well as in the results from [LM91, LMS93] about polynomial subgroup growth, M. Lazard's solution of the $p$-adic analog of Hilbert's 5th problem [Laz65] plays an important role. The result of Lazard gives a characterization of analytic pro- $p$-groups. After a long period of search, a proof of Gromov's Theorem which avoids the use of the 5th Hilbert problem was found by B. Kleiner [Kle10]. Now we will formulate a theorem (Theorem 10.3), which generalizes theorems 10.1 and 10.2, and whose proof is based on the techniques of J.S. Wilson from [Wil05, Wil] and some other results. Wilson's arguments how to handle with growth of resudually finite groups are quite original (the techniques of ultraproducts is used at some 
point), but eventully they reduce the arguments to the case of residually nilpotent groups (i.e. to the previous theorem). It would be interesting to find a proof of the theorem 10.1 which avoids the use of the $p$-adic version of Hilbert's 5th problem.

Recall that a group is called supesolvable if it has a finite normal descending chain of subgroups with cyclic quotients. Every finitely generated nilpotent group is supersolvable [Rob96], therefore the next theorem improves Theorem 10.2.

Theorem 10.3. The Gap Conjecture holds for residually supersolvable groups.

The proof of this theorem is based on the techniques of J.S. Wilson developed in [Wil05, Wil] for studies around Gap Conjecture. Currently, it is not known if the main Conjecture holds for residually polycyclic and, more generally, residually solvable groups. However, it is quite plausible that it does.

Conjecture 3. (Gap Conjecture with parameter $\beta$ ) There exists $\beta, 0<\beta<1$, such that if the growth function $\gamma_{G}(n)$ of a finitely generated group $G$ is strictly bounded from above by $e^{n^{\beta}}$ (i.e. if $\left.\gamma(n) \prec e^{n^{\beta}}\right)$, then the growth of $G$ is polynomial.

Thus the Gap Conjecture with parameter $1 / 2$ is just the Gap Conjecture (Conjecture 2). If $\beta<1 / 2$ then the Gap Conjecture with parameter $\beta$ is weaker than the Gap Conjecture, and if $\beta>1 / 2$ then it is stronger than the Gap Conjecture.

Conjecture 4. (Weak Gap Conjecture) There is a $\beta, \beta<1$ such that if $\gamma_{G}(n) \prec e^{n^{\beta}}$ then the Gap Conjecture with parameter $\beta$ holds.

As was already mentioned, there are some results of J.S. Wilson in the direction of confirmation of the Gap Conjecture. He showed that, if $G$ is a residually solvable group whose growth is strictly less than $e^{n^{1 / 6}}$, then it has polynomial growth [Wil05, Wil]. Therefore the Gap Conjecture with parameter $1 / 6$ holds for residually solvable groups. The proof of Wilson's result is based on the estimate of the rank of chief factors of finite solvable quotients of $G$. The methods of [Wil05, Wil] combined with the theorem of Morris [Mor06] and theorem of Rosset [Ros76] can be used to prove the following statement.

\section{Theorem 10.4.}

(i) The Gap Conjecture with parameter $1 / 6$ holds for left orderable groups.

(ii) The Gap Conjecture holds for left orderable groups if it holds for residually polycyclic groups.

Let us also formulate an open problem which is related to the above discussion.

\section{Problem 10.}

(i) Does there exist $\alpha, 0<\alpha<1$ such that if the growth of a finitely generated group is strictly less than the growth of $e^{n^{\alpha}}$ then it is polynomial?

(ii) If such $\alpha$ exists, what is its maximal value? Is it $<1 / 2,=1 / 2$ or $>1 / 2$ ?

(iii) In the case $\alpha$ exists (and is chosen to be maximal), is there a group (or a cancellative semigroup) with growth equivalent to $e^{n^{\alpha}}$ ?

(iv) Is there a finitely generated group approximated by nilpotent groups with growth equivalent to $e^{\sqrt{n}}$ ? Is there a residually finite-p group with growth equivalent to $e^{\sqrt{n}}$ ? ( $p$ fixed prime). 
There is some evidence based on considerations presented in the last section of this article and some additional arguments that the above conjectures and problem (parts (i), (ii), (iii)) can be reduced to consideration of three classes of groups: simple groups, branch groups and hereditary just-infinite groups. These three types of groups appear in a natural division of the class of just-infinite groups into three subclasses described in Theorem 14.3. Branch groups are defined in section 11 and a hereditary just-infinite group is a residually finite group with the property that every proper quotient of every subgroup of finite index (including the group itself) is just-infinite. In any case, the following theorem holds (observe that branch groups and hereditary just-infinite groups are residually finite groups).

Theorem 10.5. If the Gap Conjecture holds for the classes of residually finite groups and simple groups, then it holds for the class of all groups.

This theorem is a corollary of the main result of [BM07]. A different proof is suggested in [Gri12]. It is adapted to the needs of the proof of Theorem 14.6, which is discussed at the end of the article.

As it is quite plausible that the Gap Conjecture could be proved for residually finite groups (the classification of finite simple groups may help), we suspect that the validity of the Gap Conjecture depends on its validity for the class of simple groups. We will return to just-infinite groups at the end of the article and state one more reduction of the Gap Conjecture.

It is unknown if there are simple groups of intermediate growth (problem 14), but a recent article of K. Medynets and the author [GM11] shows that there are infinite finitely generated simple groups which belongs to the class LEF (locally embeddeble into finite groups); the authors conjectured that these groups are amenable. This was recently confirmed by K. Juschenko and N. Monod [JM12].

This gives some hope that groups of intermediate growth may exist within the subgroups of the groups considered in [GM11] (namely among subgroups of full topological groups [[T]] associated with minimal homeomorphisms $T$ of a Cantor set). Algebraic properties of [[T]] were studied by H. Matui [Mat06], who showed that their commutator subgroup $[[T]]^{\prime}$ is simple, and is finitely generated in the case when the homeomorphism $T$ is a minimal subshift over a finite alphabet. Observe that $[[T]]^{\prime}$ always has exponential growth, as was recently shown in [Mat11]. Therefore the only hope is that groups of intermediate growth may exist among subgroups of $[[T]]$.

There are several other Gap Conjectures related to various asymptotic characteristics of groups. We list some of them in section 12 and discuss briefly their relation to the problems and conjectures considered in this section.

\section{INTERMEDIATE GROWTH: THE UPPER AND LOWER BOUNDS}

In this section we give an overview of the main methods of getting upper and lower bounds of growth in the intermediate growth case. We begin with upper bounds. For establishing if a group is of intermediate growth, it is more important to have tools to obtain upper bounds because as soon as it is known that a group is not virtually nilpotent (and usually this is not difficult to check), its growth is known to be superpolynomial (by Gromov's theorem). Therefore, if a finitely generated infinite group possesses any property such as being simple, torsion, not residually finite, nonhopfian etc., one immediately knows that the growth is superpolynomial. 
Finding a lower bound for the growth is of interest not only because of its connection with the Gap Problem 10 discussed above, but also because of the connection with various topics in the theory of random walks on groups and spectral theory of the discrete Laplace operator.

The method for getting upper bounds for the growth in the intermediate case that we are going to describe was used in [Gri84b, Gri85a]. Roughly, the idea consists in encoding of each element $g$ of a group $G$ by a set of $d$ elements $g_{1}, \ldots, g_{d}, g_{i} \in G(d \geq 2$ and fixed) in such a way that for some fixed constants $C$ and $\lambda, 0<\lambda<1$ (independent of $g$ ) the inequality

$$
\sum_{i=1}^{d}\left|g_{i}\right| \leq \lambda|g|+C
$$

holds. The meaning of this inequality is that an element of (large) length $n$ is coded by a set of $d$ elements of the total length strictly less than $n$ (with coefficient of the reduction $\lambda$ ). In dynamics, this situation corresponds to the case when the entropy of the system is zero, while in the context of growth it corresponds to the case when the constant $\kappa$ given by (3.7) is equal to 1 (i.e. when the growth is subexponential).

There are some variations of condition (11.1). For instance, in some cases the coefficient $\lambda$ can be taken to be equal to 1 , but some additional conditions on the group have to be satisfied in order to claim that the growth is subexponential. For instance this happens in the case of the group $\mathcal{E}$, and more generally in the case of the groups $\mathcal{G}_{\omega}, \omega \in \Omega_{1}$.

At the moment, the above idea is realized only in the case of certain groups acting on regular rooted trees that are self-similar or have certain self-similarity features. The simplest property that leads to intermediate growth is the strong contracting property that we are going to describe.

The set of vertices of a $d$-regular rooted tree $T=T_{d}$ is in a natural bijection with the set of finite words over an alphabet $X$ of cardinality $d$ (usually one of the alphabets $\{0,1, \ldots, d-1\}$ or $\{1,2, \ldots, d\}$ is used). The set of vertices of the tree is graded by levels $n=0,1,2, \ldots$, and vertices of the $n$-th level are in natural bijection with the words of length $n$ (i.e. with the elements of the set $X^{n}$ ) listed in lexicographical order. Let $V=X^{*}=\bigcup_{n=1}^{\infty} X^{n}$ be the set of all vertices.

For the full group $A u t(T)$ of automorphisms of a $d$-regular rooted tree $T$, a natural decomposition into a semidirect product

$$
(\operatorname{Aut}(T) \times \cdots \times \operatorname{Aut}(T)) \rtimes \operatorname{Sym}(d)
$$

( $d$ factors) holds, as well as a corresponding decomposition of any element $g \in A u t(T)$

$$
g=\left(g_{1}, g_{2}, \ldots, g_{d}\right) \sigma .
$$

Here the element $\sigma$ of the symmetric group $\operatorname{Sym}(d)$ shows how $g$ acts on the vertices of the first level, while the projections $g_{i}$ show how $g$ acts on the subtree $T_{i}$ which has its root at vertex $i$ of the first level (we identify the subtree $T_{i}$ with $T$ using the canonical self-similarity of the regular rooted tree). In a similar way, the projection $g_{v}$ of an element $g \in A u t(T)$ can be defined for an arbitrary vertex $v \in V$.

Definition 11.1. The action of a group $G$ on a regular rooted tree $T$ is called self-similar if for any vertex $v$, the projection $g_{v}$ is again an element of $G$ modulo the canonical identification 
of the subtree $T_{v}$ with the original tree $T$. A group is called self-similar if it has a faithful self-similar action.

For instance, the groups $\mathcal{G}$ and $\mathcal{E}$ are self-similar. For generators $a, b, c, d$ of $\mathcal{G}$ the following relations of the type (11.3) hold

$$
a=(1,1) \sigma, b=(a, c) e, c=(a, d) e, d=(1, b) e,
$$

where $e$ is the identity element and $\sigma$ is a permutation (both are elements of $\operatorname{Sym}(2)$ acting on the alphabet $\{0,1\}$ ). Observe that usually the above equalities are used in the simplified form

$$
a=\sigma, b=(a, c), c=(a, d), d=(1, b) .
$$

An equivalent definition of a self-similar group is that it is a group generated by states of non-initial Mealy type automaton with the operation of composition of automata. We are not going to explain here what the Mealy automaton is, nor the group defined by it. For this approach to self-similarity, see [GNS00, Nek05]). For instance, the groups $\mathcal{G}$ and $\mathcal{E}$ are groups generated by the following automata
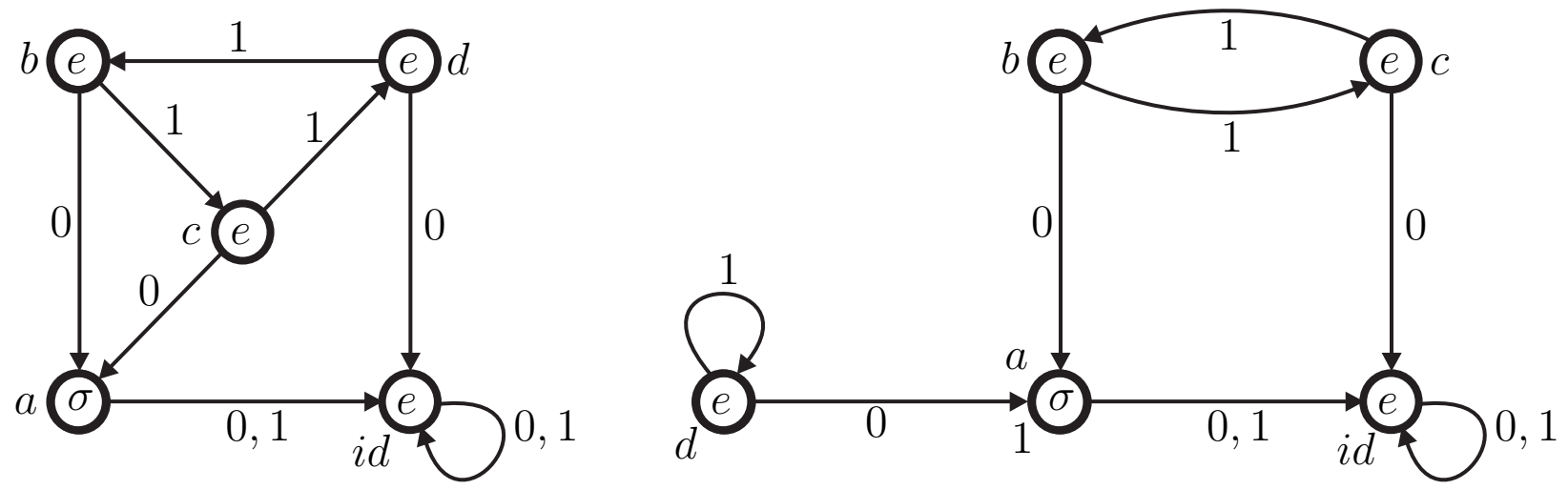

Automata generating $\mathcal{G}$ and $\mathcal{E}$

A challenging problem is to understandi what the class self-similar groups is, and especially what constitutes the subclass consisting of groups that are generated by finite automata. There is a description of groups generated by 2-state automata over the alphabet on two letters (there are 6 such groups), see [GNS00]. There are not more that 115 groups generated by 3 -state automata over an alphabet on two letters (see $\left[\mathrm{BGK}^{+} 08\right]$ where an Atlas of selfsimilar groups is started).

Another important notion useful when studying growth is the notion of a branch group, introduced in [Gri00a, Gri00b]. With each sequence $\bar{m}=\left\{m_{n}\right\}_{n=1}^{\infty}$ consisting of integers $m_{n} \geq 2$, one can associate a spherically homogeneous rooted tree $T_{\bar{m}}$ as done in [BOERT96, Gri00b, BGŚ03]. Given a group $G$ acting faithfully by automorphisms on such a tree $T=T_{\bar{m}}$ and a vertex $v \in V(T)$, the rigid stabilizer $\operatorname{rist}_{G}(v)$ is defined as the subgroup in $G$ consisting of elements which act trivially outside the subtree $T_{v}$ with its root at $v$. The rigid stabilizer rist $_{G}(n)$ of level $n$ is the subgroup generated by the rigid stabilizers of vertices of $n$th level (it is their internal direct product).

Definition 11.2. A group $G$ is called branch if it has a faithful, level-transitive action on some spherically homogeneous rooted tree $T_{\bar{m}}$ with the property that

$$
\left[G: \operatorname{rist}_{G}(n)\right]<\infty
$$


for each $n=1,2, \ldots$

This is a geometric definition of branch groups. There is also an algebraic definition discussed in [Gri00b, BGŠ03] which gives a slightly larger class of groups.

As was already mentioned in the introduction, branch groups of self-similar type are basically the only source for the constructions of groups of intermediate growth and, as is stated in the theorem 9.6, the groups $\mathcal{G}_{\omega}, \omega \in \Omega_{1}$, are branch. Branch groups constitute one of three classes in which the class of just-infinite groups naturally splits. The role of this class in the theory of growth of groups will be emphasized once more in the last section of the paper.

Definition 11.3. A finitely generated self-similar group is called contracting with parameters $\lambda<1$ and $C$ if the inequality

$$
\left|g_{i}\right|<\lambda|g|+C
$$

holds for every element $g \in G$ and any of its section $g_{i}$ on the first level.

It follows from (11.6) that

$$
\left|g_{i}\right|<|g|
$$

if $|g|>C /(1-\lambda)$. Therefore for elements of sufficiently large length the projections on the vertices of the first level are shorter.

The contracting property and self-similarity allow one to study algebraical properties of the group. This was first used in [Gri80b] to show that the groups $\mathcal{G}$ and $\mathcal{B}$ are torsion, and later many other interesting and sometimes unusual properties of the group $\mathcal{G}$ and similar type groups (for instance Gupta-Sidki p-groups [GS83]) were established.

To be more precise, the groups $\mathcal{G}_{\omega}$ are not self-similar for a typical $\omega$. But in fact, the whole family $\left\{\mathcal{G}_{\omega}\right\}_{\omega \in \Omega}$ of groups is self-similar, because the projections of any element $g \in \mathcal{G}_{\omega}$ on vertices of arbitrary level $n$ belong to the group $\mathcal{G}_{\tau^{n}(\omega)}$ where $\tau$ is the shift in the space of sequences. Moreover the contracting property holds for this family with respect to the canonical generating sets $\left\{a, b_{\omega}, c_{\omega}, d_{\omega}\right\}$. This allows one to get various results (including the estimates of the growth) using a simultaneous induction on the length of elements for infinite shift invariant families of groups from the class $\left\{\mathcal{G}_{\omega}\right\}_{\omega \in \Omega}$.

Definition 11.4. A finitely generated self-similar group acting on a $d$-regular rooted tree $T$ is called strictly contracting (or sum contracting) with parameters $k \geq 1, \lambda<1$ and $C$ if there is a level $k$ of the tree such that the inequality

$$
\sum_{v \in V_{k}}\left|g_{v}\right| \leq \lambda|g|+C
$$

holds for an arbitrary element $g \in G$ ( $V_{k}$ denotes the set of vertices of level $k$ ).

The idea of encoding of the elements of length $n$ by tuples of elements of total length less than $n$ discussed above has a clear implementation for strictly contracting groups. Namely, every element $g \in G$ can be encoded by the set $\left\{\bar{g}, g_{1}, g_{2}, \ldots, g_{d^{k}}\right\}$, where $\bar{g}$ is a shortest representative of the coset $g s t_{G}(k)$ of the stabilizer $s t_{G}(k)$ of $k$ th level, and $g_{1}, g_{2}, \ldots, g_{d^{k}}$ are projections on the vertices of $k$-th level (observe that the length of $\bar{g}$ is uniformly bounded by some constant independent of the choice of the element). 
Proposition 11.5. ([Gri84b]) Let $G$ be a strictly contracting group with parameters $k, \lambda$ and $C$. Then there is a constant $\beta=\beta(\lambda, k)<1$ such that

$$
\gamma_{G}(n) \preceq e^{n^{\beta}} .
$$

The strict contracting property holds, for instance, for the groups $\mathcal{G}_{\omega}, \omega \in \Theta$. For the groups $\mathcal{G}_{\omega}, \omega \in \Omega_{1} \backslash \Omega_{0}$, condition (11.7) does not hold in general. Regardless, a modification of the arguments allows one show that the growth is intermediate in this case as well, and even to get an upper bound of the type shown in the third part of the theorem 9.3.

Now let us turn to lower bounds. There is a much wider variety of methods used to obtain lower bounds than for upper bounds. We are going to mention the branch commensurability property, and give a short account (partly here, partly in the next section) of other methods: the anti-contracting property (which is a kind of opposite to the contracting property), the Lie method, the boundary of random walks method [Ers04a] and the Munchhausen trick method [BV05, Kai05, GN07].

The first observation is that the group $\mathcal{G}$ and many other self-similar groups of branch type, for instance the so called regularly branch groups [BGŠ03], have the property of being abstractly commensurable to some power $d \geq 2$ of itself (i.e. to a direct product of $d$ copies of the group, in the case of $\mathcal{G}$ the power $d=2$ ). Recall that two groups are said to be abstractly commensurable if they have isomorphic subgroups of finite index. Observe that there are even finitely generated groups $G$ which are isomorphic to their proper powers $G^{d}, d \geq 2$ [Jon74, BM88]. Such groups are non-hopfian (and hence not residually finite) in contrast to regularly branch groups, but among them, groups of intermediate growth have not yet been detected.

Problem 11. Does there exist a finitely generated group $G$ of intermediate growth isomorphic to some power $G^{d}, d \geq 2$ ?

For a group $G$ commensurable with $G^{d}$ there is a lower bound on growth given by the inequality

$$
e^{n^{\alpha}} \preceq \gamma_{G}(n)
$$

for some positive $\alpha$. Verifying this is an easy exercise if one keeps in mind that the growth of a group coincides with that of a subgroup of finite index, and that

$$
\gamma_{G^{d}}(n) \sim \gamma_{G}^{d}(n) .
$$

Observe that in general we do not have control over $\alpha$ in this type of argument.

Another condition that allows us to get a lower bound of type (11.7) is the following. Let $G$ be a self-similar group acting on a $d$-regular tree.

Definition 11.6. A group $G$ satisfies the anti-contracting property with parameters $k, \mu$ and $C$ if for arbitrary $g \in G$ the inequality

$$
|g| \leq \mu \sum_{v \in V_{k}}\left|g_{v}\right|+C
$$

holds, where $V_{k}$ is the set of vertices of level $k$ and $g_{v}$ is the projection of $g$ on $v$.

Inequality (11.9) is a kind of opposite to inequality (11.7). Using the methods of [Gri84b] one can prove the following fact. 
Theorem 11.7. Let $G$ be a group satisfying the anti-contracting property with parameters $\mu$ and $C$. Then there is $\alpha=\alpha(k, \mu)>0$ such that inequality (11.8) holds.

The lower bound for $\alpha$ in terms of $k$ and $\mu$ ( $C$ is not important) can be explicitly written. In the case of the group $\mathcal{G}$, the anti-contracting property holds with parameters $k=1, \mu=2$, $C=1$ and $\alpha=1 / 2$. In fact, a lower bound of type $e^{\sqrt{n}}$ holds for all groups from the family $\mathcal{G}_{\omega}, \omega \in \Omega_{1}$.

Another approach to obtain lower bounds is via an idea of W. Magnus: using Lie algebras and associative algebras associated with a group. Given a descending central series $\left\{G_{n}\right\}_{n=0}^{\infty}$ of a group $G$, one can construct the graded Lie ring $\mathcal{L}=\oplus_{n=0}^{\infty} G_{n} / G_{n+1}$ and the graded associative algebra $\mathcal{A}=\oplus_{n=0}^{\infty} A_{n}$, where $A_{n}=\Delta^{n} / \Delta^{n+1}$, and $\Delta$ is the fundamental ideal of the group algebra $\mathbb{F}[G](\mathbb{F}$ a field). The Lie operation in $\mathcal{L}$ is induced by the operation of taking commutators of pairs of elements in the group (defined first on the abelian quotients $G_{n} / G_{n+1}$ and then extended by linearity to the whole ring). The important cases are given by the lower central series and the Jennings-Lazard-Zassenhaus lower $p$-central series, which in the case of a simple field of characteristic $p$ can be defined as $G_{n}=\left\{g \in G: 1-g \in \Delta^{n}\right\}$.

By Quillen's theorem, the algebra $\mathcal{A}$ is the universal enveloping algebra of $\mathcal{L}$ (or $p$-universal in the case char $\mathbb{F}=p$ ). There is a close relationship between the growth of the algebras $\mathcal{A}$ and $\mathcal{L}$, i.e., the growth of the dimensions of the homogeneous components of these algebras. Namely, $\mathcal{L}$ has exponential growth if and only if $\mathcal{A}$ has exponential growth, and if $\mathcal{L}$ has polynomial growth of degree $d$ then $\mathcal{A}$ has intermediate growth of type $e^{n^{\alpha}}$ with $\alpha=\frac{d+1}{d+2}$ (for details see e.g., [Gri89a, BG00b]). More information about growth of algebras can be found in [KL00]. Observe that finitely generated Lie algebras may be of fractional (and even irrational) power growth as is shown in [PSZ10].

The following fact shows that the growth of $\mathcal{A}$ gives a universal lower bound for the growth of a group independently of the system of generators.

Proposition 11.8. ([Gri89a]) Let $G$ be a finitely generated group with a finite system $S$ of semigroup generators (i.e. each element of $G$ can be expressed as a product of elements from $S)$. Let $\gamma_{G}^{S}(n)$ be the growth function of $G$ with respect to $S$ and $a_{n}=\operatorname{dim}_{\mathbb{F}} \mathcal{A}_{n}$. Then, for any $n \in \mathbb{N}$,

$$
\gamma_{G}^{S}(n) \geq a_{n}
$$

If the algebra $\mathcal{L}$ is infinite dimensional, then the growth of $\mathcal{A}$ is at least $e^{\sqrt{n}}$, and therefore the growth of the group is at least $e^{\sqrt{n}}$. The Lie algebras approach was used to show that Gap Conjecture holds for residually nilpotent groups [LM91].

Observe that in order to have an example of a residually- $p$ finite group (i.e. a group approximated by finite $p$-groups) whose growth is exactly $e^{\sqrt{n}}$, the ranks of the consecutive quotients $G_{n} / G_{n+1}$ must be uniformly bounded (i.e., the group $G$ has to have bounded width). But this condition is not enough. For instance, the group $\mathcal{G}$ has finite width, as it was proved in [BG00b], but its growth is bounded from below by $e^{n^{0.51}}$. The growth of Lie algebra $\mathcal{L}$ associated with the Gupta-Sidki 3-group $\mathcal{S}$ is linear, which implies that the group $\mathcal{S}$ has growth at least $e^{n^{2 / 3}}$ (it is not known yet if Gupta-Sidki $p$-groups have intermediate growth or not). Inequality (11.10) also gives a way to prove that a group has uniformly exponential growth [Gri89a, BG00b].

Recall that a group $G$ is said to have uniformly exponential growth if

$$
\kappa_{*}=\inf _{A} \kappa_{A}>1,
$$


where $\kappa_{A}$ denotes the base of exponential growth with respect to the system of generators $A$ ( $\kappa$ is defined by relation (3.7)), and the infimum is taken over all finite systems of generators. An immediate corollary of Proposition 11.8 is

Corollary 11.9. Assume that the Lie algebra $\mathcal{L}$ associated to the group $G$ has exponential growth. Then $G$ has uniformly exponential growth.

It follows that Golod-Shafarevich groups [Gol64, GŠ64] have uniformly exponential growth.

\section{Asymptotic inVARIANTS of PROBABILISTIC AND ANALYTiS NATURE AND CORRESPONDING GAP TYPE CONJECTURES}

In this section we discuss the relation between group growth and asymptotic behavior of random walks on a group. At the end of it we formulate gap type conjectures related to the asymptotic characteristics of random walks, and discuss their relation with the growth Gap Conjecture from section 10.

The ICM paper of A. Erschler [Ers10], which we recommend to the reader, contains important material related to the subject of random walks and growth. Also we recommend the book of W. Woess [Woe00], the paper of Kaimanovich and Vershik [KV83], the article [BPS09], and the unfinished manuscript "A survey on the relationships between volume growth, isoperimetry, and the behavior of simple random walk on Cayley graphs, with examples" of C. Pittet and L. Saloff-Coste that can be downloaded from http://www. math. cornell.edu/ lsc/lau.html.

Let $G$ be a finitely generated group and $\mu$ be a probability measure on $G$ whose support $A$ generates the group. Consider a random walk $(G, \mu)$ on $G$ which starts at the identity element $e$ and the transitions $g \rightarrow g a$ take place with probability $\mu(a)$. Let $P(n)=P_{e, e}^{(n)}$ be the probability of return after $n$ steps. Observe that $P(n)=\mu^{* n}(e)$, where $\mu^{* n}$ denotes the $n$th convolution of $\mu$. In the case of a symmetric measure (i.e. when $\mu(a)=\mu\left(a^{-1}\right)$, for every $a \in A$ ) the inequality

$$
\frac{1}{P(2 n)} \leq \gamma_{G}^{A}(2 n)
$$

holds, since the maximal mass of $\mu^{* 2 n}$ is concentrated at the identity element $e$ [Woe00]. In (12.1) the probability $P(n)$ is evaluated only for even values of $n$ because for odd values it can be zero (this happens when the identity element of the group can not be expressed as a product of an odd number of generators). From now on, when we discuss the rate of decay of the probabilities $P(n)$ as $n \rightarrow \infty$, we assume that the argument $n$ takes only even values. We will use the comparison $\preceq$ of the rate of decay of $P(n)$, or the rate of growth when $n \rightarrow \infty$ of some other functions that will be introduced later, in the sense of the definition given in section 3 .

The rate of decay of the probabilities $P(n)$ can obey a power law (of the type $n^{\alpha}, \alpha<0$ ), be exponential (of type $\lambda^{n}, 0<\lambda<1$ ), or can be intermediate between the two. A power low holds if and only if the group has polynomial growth. This follows from a combination of Gromov's theorem and results of Varopoulos on random walks ([Var91] and [VSCC92, Theorem VI.5.1 on p. 84]).

An important characteristic of random walks, introduced by Kesten [Kes59b], is the spectral radius defined by relation (3.9), which in the case of symmetric measures coincides with 
the norm of the Markov operator

$$
M f(x)=\sum_{g \in G} \mu(g) f(x g) .
$$

Observe that this is also the operator given by right convolution with a measure $\mu$ acting on $l^{2}(G)$. By Kesten's criterion a group is amenable if and only if for some ("some" can be replaced by "every") symmetric measure $\mu$ whose support generates a group, the spectral radius takes its maximal possible value $r=1$ [Kes59a]. Therefore amenable groups have subexponential rate of decay of return probabilities, and the rate of decay is exponential in the case of nonamenable groups.

For groups of exponential growth, the rate of decay is not slower than $e^{-\sqrt[3]{n}}$. In other words, the upper bound

$$
P(n) \preceq e^{-\sqrt[3]{n}}
$$

holds [Var91]. This result cannot be improved, as there are groups of exponential growth for which the upper bound (12.2) is sharp (for instance, the lamplighter group $\mathcal{L}=\mathbb{Z}_{2}$ 々 $\mathbb{Z}$ or the Baumslag-Solitar solvable groups $B S(1, n), n \geq 2)$.

Because of inequality (12.1), if a bound of the type

$$
P(n) \preceq e^{-n^{\alpha}}
$$

holds, then

$$
\gamma_{G}(n) \succeq e^{n^{\alpha}}
$$

On the other hand if (12.4) holds then

$$
P(n) \preceq e^{-n^{\frac{\alpha}{\alpha+2}}}
$$

[Var91, Woe00, BPS09].

This leads to the following natural questions. What are the slowest and fastest rates of decay of probabilities $P(n)$ for groups of intermediate growth? Is it of type $e^{-n^{\alpha}}$ (respectively $e^{-n^{\beta}}$ ) for some positive $\alpha$ and $\beta$ ? What are the values of $\alpha$ and $\beta$ ? The values $1 / 2,1 / 3$ and $1 / 5$ are the first candidates for these numbers. Is there a group of intermediate growth with rate of decay $P(n) \succ e^{-n^{1 / 3}}$ ? If the rate of decay of $P(n)$ for a group of intermediate growth cannot be slower than $e^{-n^{1 / 3}}$ then the weak version of Gap Conjecture 2 holds with parameter $1 / 3$. Later we will formulate a conjecture related to the above discussion.

A new approach for obtaining lower bounds for growth based on techniques of random walks is developed by A. Erschler in [Ers04a, Ers05a]. Without getting into details, let us briefly outline some features of her approach. For a random walk given by pair $(G, \mu)$ the entropy $h=h(G, \mu)$ and the drift (or the rate of escape) $l=l(G, \mu)$ are defined as

$$
h=\lim _{n \rightarrow \infty} \frac{H(n)}{n},
$$

where $H(n)=H\left(\mu^{* n}\right)$ and $H(\mu)=-\sum_{g \in G} \mu(g) \log \mu(g)$ is Shannon entropy, and

$$
l=\lim _{n \rightarrow \infty} \frac{L(n)}{n},
$$

where $L(n)=\sum_{g \in G}|g| \mu^{* n}(g)$ is the expectation of the length $|g|$ of a random element at the $n$th moment of the random walk (the length $|g|$ is considered with respect to the 
system of generators given by the support of $\mu$ ). By the Guivarc'h inequality [Gui80], in the case of symmetric measure with finite support (or more generally with finite first moment $\left.\sum_{g \in G_{\omega}}|g| \mu(g)\right)$, the numbers $h, l$ and $\kappa$ (the base of exponential growth defined by (3.7) ) are related as

$$
h \leq l \kappa .
$$

Therefore the equality $l=0$ implies $h=0$.

An important notion due to Furstenberg is the Poisson boundary (which we will call the Poisson-Furstenberg boundary). It is a triple $(G, \mathcal{B}, \nu)$ consisting of a $G$-space $(\mathcal{B}, \nu)$ with a $\mu$-stationary probability measure $\nu$ (i.e. $\mu * \nu=\nu$ ). This boundary describes space of bounded $\mu$-harmonic functions:

$$
f(g)=\int_{\mathcal{B}} \phi(g x) d \nu(x)
$$

(the Poisson integral). The left hand side of the last equality takes values in the space of bounded $\mu$-harmonic functions while $\phi$ belongs to the space $L^{\infty}(\mathcal{B})$ (see [KV83] for details). The Liouville property of a group (more precisely, of a pair $(G, \mu)$ ) is that every bounded $\mu$-harmonic function is constant; this property is equivalent to the triviality of the PoissonFurstenberg boundary.

The entropy criterion due to Avez-Derriennic-Kaimanovich-Vershik [KV83] states the following. Let $G$ be a countable group and $\mu$ a probability measure on $G$ with finite entropy $H(\mu)$. Under this assumption the Poisson-Furstenberg boundary is trivial if and only if the entropy $h$ of the random walk is equal to zero.

If $\mu$ is symmetric and has finite first moment with respect to some (and hence with respect to every) word metric on $G$, then the entropy $h$ is positive if and only if the rate of escape $l$ of the random walk determined by $(G, \mu)$ is positive. In one direction this follows from the Guivarc'h inequality (12.8). The converse was proved by Varopoulos [Var91] for finitely supported measures and then extended by Karlsson and Ledrappier to the case of a measure with finite first moment [KL07]. It is known that for a group of intermediate growth and a measure with finite first moment, the entropy is zero; therefore the drift is also zero and the Poisson-Furstenberg boundary is trivial. The vanishing of the entropy, and hence the triviality of the boundary, can easely be deduced for instance from inequality (18) in [KV83]. In the case of a nonsymmetric measure, the drift $l$ can be nonzero, even on a group of polynomial growth (for instance, for a $(p, 1-p), p<1 / 2$ random walk on $\mathbb{Z}$ ). But, in the case of a symmetric measure, it is zero if and only if $h=0$. Therefore, in the case of groups of subexponential growth and symmetric measures with finite first moment $h=l=0$ and the functions $H(n)$ and $L(n)$ grow sublinearly. The Poisson-Furstenberg boundary is also trivial for each group of polynomial growth and any measure $\mu$; this follows from Gromov's result on groups of polynomial growth and the theorem of Dynkin and Malyutov concerning Martin boundaries of nilpotent groups (from which the triviality of the Poisson-Furstenberg boundary follows in this case) [DM61] (see also the work of G. Margulis [Mar66]).

To obtain a lower bound for growth for some groups from the family $G_{\omega}$ and to obtain new results about the Poisson-Furstenberg boundary of random walks on groups of intermediate growth, in [Ers04a] Erschler introduced the so called "strong condition" (*) for some type actions on the interval $(0,1]$. She proved that if a group $G$ satisfies condition $(*)$ and the group $\operatorname{germ}(G)$ of germs of $G$ (also defined in [Ers04a]) satisfies some extra condition, then $G$ 
admits a symmetric probabilistic measure $\mu$ with finite entropy $H(\mu)$ and nontrivial PoissonFurstenberg boundary. These conditions are satisfied for all groups $G_{\omega}, \omega \in \Omega_{4}$, where the set $\Omega_{4}=\Omega_{1} \backslash \Omega_{0}$ consists of the sequences containing only two symbols from $\{0,1,2\}$, with each of them occuring infinitely many times in the sequence. Recall that by Theorem 9.6 all such groups have intermediate growth. The groups $G_{\omega}, \omega \in \Omega_{4}$ are first examples of groups of intermediate growth possessing a symmetric measure with nontrivial Poisson-Furstenberg boundary. Also for all these groups and any $\epsilon>0$, a lower bound on growth of type

$$
\exp \frac{n}{\log ^{2+\epsilon} n} \preceq \gamma_{\mathcal{G}_{\omega}}(n)
$$

holds [Ers04a]. The proof of this result uses the existence of a special element $g \in G$ of infinite order. It is based on the combination of facts of existence of measure with nontrivial Poisson boundary and the analogue of inequality (12.8) for measures with infinite first moment.

Interesting results concerning growth and triviality of the Poisson-Furstenberg boundary were obtained by Karlsson, Ledrappier and Erschler [KL07, EK10]. These results led to upper bounds on growth of $H(n)$ and $L(n)$ for the groups $G_{\omega}$, and show that, under certain conditions, non vanishing of the drift implies that the group is indicable (i.e. existence of surjective homomorphism onto $\mathbb{Z}$ ).

Let us mention now a new method of studying of asymptotic properties of self-similar groups discovered by L. Barthlodi and B. Virag in [BV05]. It received further development in the paper of V. Kaimanovich [Kai05], where it was called the Munchhausen trick, and in the papers of Bartholdi, Kaimanovich and Nekrashevych [BKN10], and of Amir, Angel and Virag [AAV09]. In [Kai05] the entropy arguments were used to prove amenability, the notion of a self-similar measure was introduced, and the map $\psi$ in the space of probabilistic measures on a self-similar group was defined. It allows one to describe self-similar measures as fixed points of this map. The relation between $\psi$ and the classical tool of linear algebra known as the Schur complement was established in [GN07].

The Munchhausen trick has been used to prove amenability of certain self-similar groups of exponential growth [BV05, BKN10, AAV09]. For the first time, this method was applied to prove the amenability of the group $B$, named "Basilica", which was introduced in the paper of A. Zuk and the author [GZ̈02], and can be defined as the iterated monodromy group of the polynomial $z^{2}-1$, or alternatively as the group generated by the automaton $\mathcal{A}_{852}$ from the Atlas of self-similar complexity $(2,3)$ groups $\left[\mathrm{BGK}^{+} 08\right]$. Observe that $B$ has exponential growth. The amenability of $B$ allows us to separate the class $A G$ from the class $S G$ of subexponentially amenable groups that was mentioned in section 7 . It was originally defined in [Gri98] (where the question about the possible coincidence of classes $A G$ and $S G$ was raised). It is currently unknown whether the cardinality of the set $A G \backslash S G$ is the cardinality of the continuum or not.

Unfortunately, the Munchausen trick has not been used so far to obtain new information on growth of groups. But it was used in [BV05, Kai05, BKN10] to obtain interesting results about the rate of growth of the functions $H(n), L(n)$, and the rate of decay of $P(n)$. For instance, in the case of the Basilica group, $P(n) \succeq e^{-n^{2 / 3}}$ [BV05], while for the group of intermediate growth $\mathcal{G}$, the lower bound is $P(n) \succeq e^{-n^{1 / 2-\epsilon}}$ for any positive number $\epsilon$ (this 
follows for instance from the results of the paper by Bartholdi, Kaimanovich and Nekrasevych [BKN10]). Using inequalities (12.2) ) and (12.5) we obtain the following estimates:

$$
\begin{gathered}
e^{-n^{2 / 3}} \preceq P_{B}(n) \preceq e^{-n^{1 / 3}}, \\
e^{-n^{1 / 2-\epsilon}} \preceq P_{\mathcal{G}}(n) \preceq e^{-n^{1 / 5}} .
\end{gathered}
$$

It would be interesting to find the asymptotics of the rate of decay of $P(n)$ for each of the groups $\mathcal{B}$ and $\mathcal{G}$ (as well as the rate of growth of the functions $H(n)$ and $L(n)$ ).

Behind the idea of the Munchausen trick is the conversion of the self-similarity of the group into self-similarity of the random walk on the group. Let $G$ be a self-similar group acting level transitively on a $d$-regular rooted tree $T_{d}$, and let $\mu$ be a probability measure on $G$. Denote by $H=s t_{G}(x)$ the stabilizer of a vertex $x$ on the first level. Then $[G: H]=d$. Let $p_{x}: H \rightarrow G$ be the projection homomorphism of $H$ on the subtree $T_{x}$ with root $x\left(p_{x}(g)=g_{x}\right.$, where $g_{x}$ is the section of $g$ at vertex $x$ ), and let $\mu_{H}$ be the probability distribution on $H$ given by the probability of the first hit of $H$ by a random walk on $G$ determined by $\mu$. Denote by $\mu_{x}=\left(p_{x}\right)_{*}\left(\mu_{H}\right)$ the image of the measure $\mu_{H}$ under the projection $p_{x}$.

Definition 12.1. A measure $\mu$ is called self-similar if for some vertex $x$ of the first level

$$
\mu_{x}=(1-\lambda) \delta_{e}+\lambda \mu
$$

for some $\lambda, 0<\lambda<1$.

Theorem 12.2. ([BV05, Kai05]) If a self-similar group $G$ possess a self-similar symmetric probability measure $\mu$ with finite entropy and contracting coefficient $\lambda, 0<\lambda<1$, then the entropy $h$ of the corresponding random walk on $G$ is zero and therefore the group $G$ is amenable.

For instance, for $\mathcal{G}$ the measure $\mu=\frac{4}{7} a+\frac{1}{7}(b+c+d)$ is self-similar with contracting coefficient $\lambda=1 / 2$ [Kai05].

Let $G$ be an amenable group with a finite generating system $A$. Then one can associate with $(G, A)$ a function

$$
F_{G}(n)=\min \left\{n: \text { there is a finite } \operatorname{subset} F \subset G \text { s.t. } \frac{|F \triangle a F|}{|F|}<\frac{1}{n}, \forall a \in A\right\},
$$

which is called the Fölner function of $G$ with respect to $A$ (because of Fölner's criterion of amenability [Føl57]). This function was introduced by Vershik in the Appendix to the Russian edition of Greenleaf's book on amenability [Gre69]. The growth type of this function does not depend on the generating set. By a result of Coulhon and Saloff-Coste [CSC93], the growth function and the Fölner function are related by the inequality

$$
\gamma(n) \preceq F(n) .
$$

On the other hand, for groups with $\gamma(n) \preceq e^{n^{\alpha}}$, the Fölner function can be estimated as in [Ers06, Lemma 3.1]

$$
F(n) \preceq e^{n^{\frac{\alpha}{1-\alpha}}} .
$$

It is proved by Erschler that for an arbitrary function $f: \mathbb{N} \rightarrow \mathbb{N}$ there is a group of intermediate growth with Fölner function $F(n) \succeq f(n)$. The method of construction of such groups is based on the "oscillation" type techniques that we discuss briefly in the last section. Very interesting results about the asymptotics of random walks on Schreier graphs 
associated with finitely generated groups, and in particular with $\mathcal{G}$, are obtained by Erschler in [Ers05a]. We shall reformulate the following question soon in the form of a conjecture.

Problem 12. Can a Fölner function grow strictly slower than the exponential function but faster than any polynomial?

In view of the inequality (12.12), the Gap Conjecture holds if the answer to the last problem is negative.

The next conjecture (consisting of three subconjectures) was formulated by P. Pansu and the author in 2000 in an unpublished note. Let $G$ be an amenable finitely generated group, $\mu$ a symmetric probability measure with finite support that generates $G, M$ a Markov operator of the associated random walk on $G$ given by $\mu$, and $P(n)$ the probability of return after $n$ steps for this random walk. Let $F(n)$ be the Fölner function, and $\mathcal{N}(\lambda)$ be the spectral density defined by the relation

$$
\mathcal{N}(\lambda)=t r_{v N}\left(\chi_{(-\infty, \lambda]}(\Delta),\right.
$$

where $t r_{v N}$ is the von Neumann trace defined on the von Neumann algebra $\mathcal{N}(G)$ of $G$, generated by the right regular representation, $\Delta=I-M \in \mathcal{N}(G)$ is the discrete Laplace operator on $G$, and $\chi_{(-\infty, \lambda]}(\Delta)$ is the projection obtained by application of the characteristic function $\chi_{(-\infty, \lambda]}$ to $\Delta$. We are interested in the asymptotic behavior of $P(n)$ and $F(n)$ when $n \rightarrow \infty$ and of $\mathcal{N}(\lambda)$ when $\lambda \rightarrow 0+$. Their asymptotic behavior does not depend on the choice of the measure $\mu$ [BPS09].

\section{Conjecture 5 .}

(i) (Gap conjecture for the heat kernel). The function $P(n)$ is either of power rate of decay or satisfies

$$
P(n) \preceq e^{-\sqrt[3]{n}}
$$

(ii) (Gap Conjecture for the Fölner function) The Fölner function $F(n)$ has either polynomial growth or the growth is at least exponential.

(iii) (Gap Conjecture for the spectral density) The spectral density $\mathcal{N}(\lambda)$ either has power decay of type $\lambda^{d / 2}$ for some $d \in \mathbb{N}$ when $\lambda \rightarrow 0$ or

$$
\mathcal{N}(\lambda) \preceq e^{-\frac{1}{\sqrt[4]{\lambda}}}
$$

Let us also formulate a modified version of the previous conjecture. We guess that for each of three conjectures stated below there is a number $\beta>0$ for which it holds.

Conjecture 6. (Gap Conjectures with parameter $\beta, \beta>0$ )

(i) The function $P(n)$ is either of power rate of decay or satisfies

$$
P(n) \preceq e^{-n^{\beta}} .
$$

(ii) The Fölner function $F(n)$ has either polynomial growth or the growth is not less than $e^{n^{\beta}}$

(iii) The spectral density $\mathcal{N}(\lambda)$ either has power decay of type $\lambda^{d / 2}$ for some $d \in \mathbb{N}$ or

$$
\mathcal{N}(\lambda) \preceq e^{-\lambda^{-\beta}} .
$$


Each of the above alternatives separates the case of polynomial growth from the intermediate growth case.

Perhaps gap type conjectures can also be formulated in a reasonable way for some other asymptotic characteristics of groups such as the isoperimetric profile, $L^{2}$-isoperimetric profile, entropy function $H(n)$, drift function $L(n)$, etc.

There are relations between all of the conjectures stated here to the growth Gap Conjecture from section 10 (which we will call here Growth Gap Conjecture), and to its generalization, Conjecture 3). For instance, using inequality (12.12) we conclude that the Gap Conjecture for the Fölner function implies the Growth Gap Conjecture. On the other hand, inequality 12.11 shows that the Growth Gap Conjecture with parameter $\beta$ implies the Gap Conjecture with parameter $\beta$ for the Fölner function. Therefore the Weak Gap Conjecture for growth is equivalent to the Weak Gap Conjecture for the Fölner function (the latter is formulated similarly to the conjecture 4).

The Growth Gap Conjecture with parameter $\alpha$ implies the Gap Conjecture with parameter $\frac{\alpha}{\alpha+2}$ for the return probabilities $P(n)$ (because of inequality (12.5)) etc. It would be interesting to find the relation between all stated conjectures.

The results of [Gri89a, LM91, Wil] and some statements from this article provide the first classes of groups for which the Gap Conjectures of the given type hold. For instance, the Gap Conjecture with parameter $\frac{1}{5}$ for return probabilities $P(n)$, and with parameter $\frac{1}{2}$ for the Fölner function $F(n)$ hold for residually supersolvable groups.

\section{INVERSE ORBIT GROWTH AND EXAMPLES WITH EXPLICIT GROWTH}

Until recently there was no exact computation of the intermediate growth in the sense of the Schwarz-Milnor equivalence. The first such examples were produced recently by L. Bartholdi and A. Erschler [BA10]. The idea is very nice and we shall explain it briefly.

Observe that the notion of growth can be defined for transitive group actions. Namely, if a finitely generated group $G$ with a system of generators $A$ acts transitively on a set $X$ and a base point $x \in X$ is selected, then the growth function $\gamma_{X, x}^{A}(n)$ counts the number of points in $X$ that can be reached from $x$ by consecutive applications of at most $n$ elements from set $A \cup A^{-1}$. The growth type of this function (in the sense of the equivalence $\sim$ ) does not depend on the choice of $x$. The triple $(G, X, x)$ can be encoded by the Schreier graph (or the graph of the action) $\Gamma$, with set of vertices $X$ and a set of oriented edges (labeled by the elements of $A$ ) consisting of pairs $(x, a(x)), x \in X, a \in A$. The growth function of the action is the same as the growth function of the graph $\Gamma$, which is a $2 d$-regular graph (viewed as non-oriented graph).

It is easy to construct actions with intermediate growth between polynomial and exponential. An interesting topic is the study of growth of Schreier graphs associated with actions of self-similar groups on corresponding rooted trees and their boundaries. The graphs we allude to here are of the form $\Gamma=\Gamma(G, H, A)$, where $G$ is a finitely generated self-similar group with generating set $A$, and $H=S t_{G}(\xi)$, where $\xi$ is a point on the boundary of the tree. Such graphs are isomorphic to the corresponding graphs of the action of the group on the orbit of the base point (namely $\xi$ ). For contracting groups they have polynomial growth, which can be of fractional and even irrational degree [BG00a, Bon07]. There are examples of actions with quite exotic intermediate behavior like $n^{\log ^{m} n}$ for some $m>0$ [BH05, GŠ06, Bon11, BCSDN11]. At the same time there are examples with quite regular polynomial type orbit growth. For instance, for the group $\mathcal{G}$ the orbit growth is linear. 
Schreier graphs of the action of $\mathcal{G}$ on the first three levels of the binary tree are shown in Figure 13.1 and the infinite graph of the action on the orbit of a typical point of the boundary
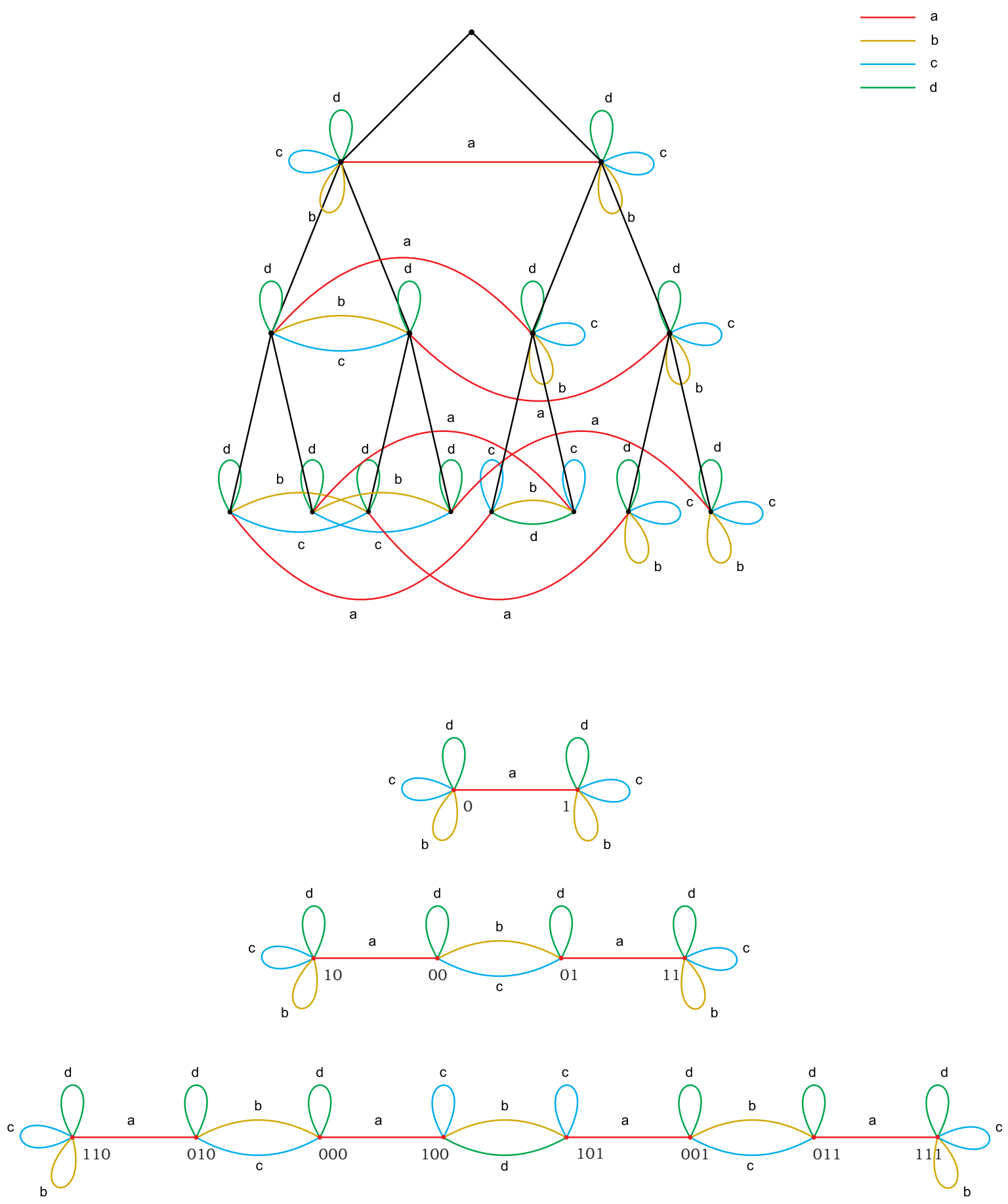

FiguRE 13.1. The action of $\mathcal{G}$ on the first three levels of the tree.

is given in Figure 13.2 (drawn in two versions: with labels and without).

In [BA10, BE11] the notion of inverted orbit growth function has been introduced. Let us briefly explain the idea and formulate some of the results of these articles. Let $G$ be a 


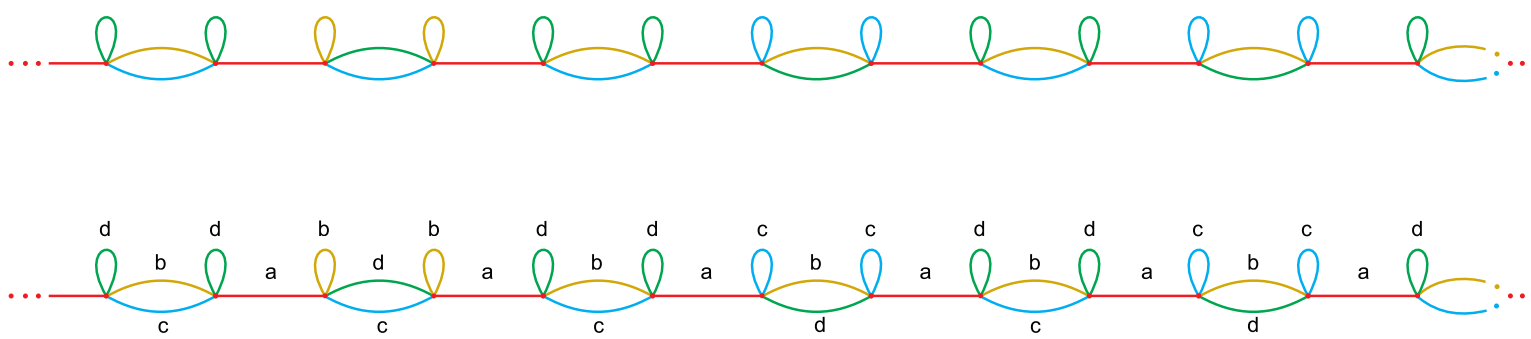

Figure 13.2. Typical Schreier graph of the boundary action.

group acting on the right on a set $X, S$ be a generating set for $G$ (viewed as monoid), and let $x \in X$ be a base point. Denote by $S^{*}$ the set of (finite) words over the alphabet $S$. For a word $w=w_{1} \ldots w_{l} \in S^{*}$, its inverted orbit is

$$
\mathcal{O}(w)=\left\{x, x w_{l}, x w_{l-1} w_{l}, x w_{1} \ldots w_{l-1} w_{l}\right\},
$$

and the inverted orbit growth of $w$ is $\delta(w)=|\mathcal{O}(w)|$. The inverted orbit growth function of $G$ is the function

$$
\Delta(n)=\Delta_{(G, X, x)}(n)=\max _{w}\{\delta(w)|| w \mid=n\} .
$$

Clearly $\Delta_{(G, X, x)}(n) \preceq \gamma_{(G, X, x)}(n)$.

The notions of wreath product and permutational wreath product are standard in group theory. Consider groups $A, G$ and a $G$-set $X$, such that $G$ acts on $X$ on the right. The wreath product $W=A \imath_{X} G$ is a semidirect product of $\sum_{X} A$ (a direct sum of copies of $A$ indexed by $X$ ) with $G$ acting on $\sum_{X} A$ by automorphisms induced by the corresponding permutations of $X$. In other words, view elements of $\sum_{X} A$ as finitely supported functions $X \rightarrow A$. A left action of $G$ on $\sum_{X} A$ by automorphisms is then defined by $(g f)(x)=f(x g)$. There are two versions of (permutational) wreath products, restricted and unrestricted, and here we use the restricted version (because of the assumption of finiteness of the support of the elements from the base group $\left.\sum_{X} A\right)$.

In the next two theorems, proved in [BA10], $X$ is a $\mathcal{G}$-orbit of an arbitrary point of the boundary $\partial T$ of the binary rooted tree $T$ that does not belong to the orbit of the point $1^{\infty}$. Observe that the orbit of $1^{\infty}$ consists of the sequences cofinal to $1^{\infty}$, where two sequences are cofinal if they coincide starting with some coordinate, and more generally, two points of the boundary of $T$ are in the same orbit if they are cofinal [BG00a, BG02a, Gri05]. The graph of the action of $\mathcal{G}$ (after deletion of the labels) looks similar to the one shown in the last figure. Recall that the number $\alpha_{0} \approx 0.7674$ was defined above and is included in (9.2).

Theorem 13.1. Consider the following sequence of groups: $K_{0}=\mathbb{Z} / 2 \mathbb{Z}$, and $K_{k+1}=$ $K_{k} 2_{x} \mathcal{G}$. Then every $K_{k}$ is a finitely generated infinite torsion group, with growth function

$$
\gamma_{K_{k}}(n) \sim \exp \left(n^{\left.\left[1-\left(1-\alpha_{0}\right)^{k}\right)\right]}\right) .
$$

Recall that the torsion free group of intermediate growth $\hat{\mathcal{G}}$, constructed in [Gri85a], was mentioned in section 9 (theorem 9.3).

Theorem 13.2. Consider the following sequence of groups: $H_{0}=\mathbb{Z}, H_{1}=\hat{\mathcal{G}}$ and $H_{k+1}=$ $H_{k} \imath_{X} \mathcal{G}, k \geq 1$. Their growth functions satisfy

$$
\gamma_{H_{k}}(n) \sim \exp \left(\log (n) n^{1-\left(1-\alpha_{0}\right)^{k}}\right) .
$$


It is remarkable that not only two infinite series of groups of intermediate growth with precisely computed growth have been constructed, but also that the precise growth of the torsion free group $\hat{\mathcal{G}}$, constructed more than 25 years ago, has finally been evaluated.

The proof of these two theorems is based on the calculation of the inverse orbit growth of the action of $\mathcal{G}$ on $X$, which is $n^{\alpha_{0}}$ in this case. In the realization of the first step of this program the technique used previously by Bartholdi [Bar98] for improving the upper bound in the case of the group $\mathcal{G}$ from the value $\log _{32} 31$ used in [Gri84b] to the value $\alpha_{0}$ established in [Bar98, MP01a] is explored again. The technique is based on assigning positive weights to the canonical generators $a, b, c, d$ and finding the values that give the best possible (for this approach) upper bound for growth.

The above ideas and some other tools (in particular, the dynamics of partially continuous self-maps of simplices) has been used by Bartholdi and Erschler in [BE11] to construct a family of groups with growth of the type $e^{n^{\alpha}}$ with $\alpha$ belonging to the interval $\left(\alpha_{0}, 1\right)$. Moreover, they presented the following impressive result. Let $\eta_{+} \approx 2.4675$ be the positive root of $x^{3}-x^{2}-2 x-4$.

Theorem 13.3. Let $f: \mathbb{R} \rightarrow \mathbb{R}$ be a function satisfying

$$
f(2 x) \leq f(x)^{2} \leq f\left(\eta_{+} x\right)
$$

for all $x$ large enough. Then there exists a finitely generated group with growth equivalent to the growth of $f$.

This theorem provides a large class of growth functions of finitely generated groups that "fill" the "interval" $\left[e^{n^{\alpha} 0}, e^{n}\right]$.

\section{Miscellaneous}

Recall that a group has uniformly exponential growth if it has exponential growth and moreover the number $\kappa_{*}$ (the base of exponential growth) defined by (11.11) is $>1$.

In [Gro81b] Gromov raised the following question.

Gromov's Problem on Growth (II). Are there groups of exponential but not uniformly exponential growth?

Some preliminary results concerning uniformly exponential growth were obtained by P. de la Harpe [dlH02]. The problem of Gromov (II) was solved by J.S. Wilson [Wil04b, Wil04a] by providing an example of such a group. A shorter solution was found later by Bartholdi in [Bar03b]. Tree-like constructions and techniques of self-similar groups naturally leading to such examples were explored by V. Nekrashevych [Nek10]. Similar to the intermediate growth case, all known examples of groups of exponential but not uniformly exponential growth are based on the use of self-similar groups of branch type.

There are results that show that groups within certain classes of groups of exponential growth are of uniformly exponential growth. This holds, for instance, for hyperbolic groups (M. Koubi [Kou98]), one-relator groups, solvable groups, linear groups over fields of characteristic 0 (A. Eskin, S. Mozes and H. Oh [EMO05]), subgroups of the mapping class group which have exponential growth (J. Mangahas [Mang10]), and some other groups and classes of groups.

The fact that solvable groups of exponential growth have uniformly exponential growth was proved by D. Osin [Osi03] and he generalized this result to elementary amenable groups 
[Osi04]. E. Breuillard gave a "ping-pong" type proof of Osin's result [Bre07]. A connection between the so called "slow growth" and the Lehmer conjecture is another result in Breuillard's paper. The fact that one relator groups of exponential growth have a uniformly exponential growth is proved by P. de la Harpe and the author [GdlH01], and that a one relator group has either polynomial growth or exponential growth is shown in the paper of T. Ceccherini-Silberstein and the author [CSG97].

A second topic of this section is the oscillation phenomenon that exists in the world of growth of finitely generated groups. The meaning of this is that there are groups whose growth in a certain sense may oscillate between two types of growth. This was first discovered by the author in [Gri84b] and was further developed in his Habilitation [Gri85b]. The goal achieved in that work was the construction of a chain and an anti-chain of cardinality of the continuum in the space of growth degrees of finitely generated groups. The "trick" used for this purpose can be described briefly as follows.

The groups $\mathcal{G}_{\omega}, \omega \in \Omega \backslash \Omega_{1}$ (recall that $\Omega \backslash \Omega_{1}$ consists of sequences that are constant at infinity) are virtually abelian, while the rest of the groups $\mathcal{G}_{\omega}, \omega \in \Omega_{1}$ have intermediate growth and the set $\left\{\mathcal{G}_{\omega}, \omega \in \Omega_{1}\right\}$ has the property that if two sequences $\lambda, \mu \in \omega \in \Omega_{1}$ have the same prefix of length $n$ then the subgraphs with vertices in the balls $B_{\mathcal{G}_{\lambda}}\left(2^{n-1}\right)$ and $B_{\mathcal{G}_{\nu}}\left(2^{n-1}\right)$ of radius $2^{n-1}$ with centers at the identity elements in the Cayley graphs of these groups are isomorphic. It was suggested in [Gri84b] to replace the groups from the set $\left\{\mathcal{G}_{\omega}, \omega \in \Omega \backslash \Omega_{1}\right\}$ by the set of accumulation points of $\left\{\mathcal{G}_{\omega}, \omega \in \Omega_{1}\right\}$ in the space of the Cayley graphs of 4-generated groups, supplied with a natural topology introduced in the article. Then each of deleted groups $\left\{\mathcal{G}_{\omega}, \omega \in \Omega \backslash \Omega_{1}\right\}$ is replaced by some virtually metabelian group (for which we will keep the same notation) of exponential growth and the modified set of groups $\left\{\mathcal{G}_{\omega}, \omega \in \Omega\right\}$ becomes a closed subset in the space of groups homeomorphic to a Cantor set.

Consider a sequence $\theta$ of the type

$$
\theta=(012)^{m_{1}} 0^{k_{1}}(012)^{m_{2}} 0^{k_{2}} \ldots
$$

with a very fast growing sequence $m_{1}, k_{1}, m, k_{2}, \ldots, m_{i}, k_{i}, \ldots$ of parameters. In view of the property that the balls of radius $2^{n-1}$ coincide for the considered groups when they are determined by sequences with the same prefix of length $n$, and the fact that the group $\mathcal{G}_{\omega}, \omega \in$ $\Omega_{1}$ is (abstractly) commensurable to the group $\mathcal{G}_{\tau^{n}(\omega)}^{2^{n}}(\tau$ the shift in space of sequences), we conclude that for initial values of $n$ when $n \in\left[1, R\left(m_{1}\right)\right]\left(R\left(m_{1}\right)\right.$ determined by $\left.m_{1}\right)$ the group $\mathcal{G}_{\theta}$ behaves as a group of intermediate growth (similar to $\mathcal{G}=\mathcal{G}_{(012) \infty}$ ), but then for larger values of $n, n \in\left[R\left(m_{1}\right)+1, R\left(m_{1}, k_{1}\right)\right]\left(R\left(m_{1}, k_{1}\right)\right.$ determined by $\left.m_{1}, k_{1}\right)$ it starts to grow as the group $\mathcal{G}_{0^{\infty}}^{3 m_{1}-1}$ (i.e. exponentially), but then again the growth slows down and behaves in intermediate fashion, etc. Taking sequences of the type (14.1) but determined by various sequences of parameters, one can construct a chain and an anti-chain of cardinality of the continuum in the space of growth degrees of 3-generated groups; this was done in [Gri84b, Gri85b].

The possibility of application of the oscillation technique is based on the use of a topology in the space of Cayley graphs (or what is the same, in the space of marked groups) introduced in [Gri84b] (this topology is a relative to Chabauty topology known in the theory of locally compact groups and geometric topology [dlH00]). The space of marked groups is a compact totally disconnected space and one of the major problems is to find its Cantor-Bendixson 
rank (for more on this and related problems see [Gri05]). Although the fact that this space has a nontrivial perfect core follows from the result established by B.Neumann in 1937 (a construction of uncountably many 2-generated groups, up to isomorphism), there is a considerable interest in finding Cantor subsets in the space of marked groups consisting of interesting families of groups. The first such subset was identified in [Gri84b], which, with exception of a countable set, consists of groups of intermediate growth $\mathcal{G}_{\omega}, \omega \in \Omega_{1}$ (its construction was described in section 9).

Oscillation techniques received further development in the paper of Erschler [Ers06] (which was already mentioned at the end of section 12), where the notion of piecewise automatic group was introduced. Using this notion, Erschler constructed groups of intermediate growth with arbitrary fast growth of the Fölner function. Moreover, the asymptotic entropy of a random walk on Erschler groups can be arbitrarily close to a linear function, while at the same time, the Poisson boundary can be trivial. Oscillation techniques, in combination with ideas from [BA10] were used also by J. Brieussel [Bri11]. The most recent result of "oscillation character" is due to M. Kassabov and I. Pak [KP11]. They demonstrated a very unusual oscillation phenomenon for groups of intermediate growth and their result is based on a new idea which we are going to explain briefly.

The groups $G_{\omega}$, as well as groups of branch type, act on spherically homogeneous rooted trees, that is, trees $T_{\bar{k}}$ defined by sequences of integers $\bar{k}=k_{1}, k_{2}, \ldots$, with $k_{i} \geq 2$; these sequences $\bar{k}$ are called the branch index ( $k_{i}$ is the branching number for the $i$ th level of the tree). Kassabov and Pak suggested to modify this approach by considering actions on decorated trees by attaching to some levels of $T_{\bar{k}}$ finite subtrees with actions of suitably chosen finite groups (each vertex of the corresponding level is decorated by the same structure). The sequence $\left\{F_{i}\right\}$ of attached groups has to satisfy certain properties (in particular the groups need to be generated by four involutions, three of which commute as in case of $\mathcal{G}_{\omega}$ ), but the main property is that the groups $F_{i}$ must behave as expanders, in a certain sense. Namely, their Cayley graphs have to have diameters $d_{i}$ growing as $i \rightarrow \infty$ as the logarithm of the size of the group, and for values of $n$ in the range $1 \leq n \leq C d_{i}(C, 0<C<1$ some constant independent of $i$ ), the growth functions $\gamma_{i}(n)$ have to behave as the exponential function.

As it was already mentioned in the introduction, there are various types of asymptotic characteristics that can be associated with algebraic objects. In addition to the group growth, and other characteristics considered in section 12, the subgroup growth, the conjugacy growth, the geodesic growth, and many other types of growth have been studied. The subgroup growth was already discussed a little bit and we refer the reader to the comprehensive book on this and other subjects, by Lubotzky and Segal [LS03] and the literature cited therein.

The conjugacy growth counts the number of conjugacy classes of length $\leq n, n=1,2, \ldots$, where the length of a conjugacy class is the length of the shortest representative of this class. Interesting results on this subject are obtained by I. Babenko [Bab88] (who was perhaps the first who introduced this notion), M. Coornaert and G. Knieper, who studied the hyperbolic groups case [CK02], Breuillard and Coornaert [BdC10] (the case of solvable groups), and M. Hull and D. Osin [HO11] who showed that basically any monotone function growing not faster than an exponential function is equivalent to the conjugacy growth function. This is a far from complete list of papers and results on this subject (for a more complete list see the literature in the cited papers). 
The geodesic growth of a pair $(G, A)$ (a group and a finite system of generators) is a growth of the language of geodesic words over alphabet $A \cup A^{-1}$ (i.e. words over an alphabet of generators that represent geodesics in the Cayley graph $\Gamma(G, A)$ with the origin at the identity element). It can be polynomial with respect to some system of generators but exponential with respect to other systems of generators, and it is unclear if it can be intermediate between polynomial and exponential. This notion was studied in [Can84, NS95, GN97, BBES11]. The following question was discussed by M. Shapiro and the author around 1993.

Problem 13. Are there pairs $(G, A)$ consisting of a group of intermediate growth $G$ and a finite system of generators $A$ with intermediate geodesic growth?

All known groups of intermediate growth have exponential geodesic growth. For instance for $\mathcal{G}$ this follows from the fact that the Schreier graph presented in figure 13.2 has exponential geodesic growth which is obvious.

The study of geodesic growth is a particular case of study of growth of formal languages. Such questions originated in the work of Schützenberger in the 1950s. The literature on this subject related to group theory can be found in[BG02b, CSW02, CSW03, Gil05]. Observe that for regular and for context-free languages, growth can be only polynomial or exponential (R. Incitti [Inc01], M. Bridson and R. Gilman [BG02b]), while the intermediate type behavior is possible for indexed languages (the next in the language hierarchy type of languages after the context-free languages), as is shown in the note of A. Machi and the author [GM99].

There are interesting studies about growth of regular graphs. One of the first publications on this subject is the article of $\mathrm{V}$. Trofimov [Tro84] where, under certain conditions on the group of automorphisms of the graph, the case of polynomial growth is studied. In the last decade, the study of Schreier graphs of finitely generated groups, and in particular of their growth and amenability properties has been intensified. Some results were already mentioned in previous sections. We mention here the paper of Bartholdi and the author [BG00a], where it is observed that Schreier graphs of self-similar contracting groups are of polynomial growth, and that in this case the degree of the polynomial (more precisely of power) growth can be non-integer and even a transcendental number. Interesting results on the growth of Schreier graphs are obtained by I. Bondarenko [Bon07] (see also [BH05, BCSDN11]). The amenability of Schreier graphs associated with actions of almost finitary groups on the boundary of rooted tree is proven in [GN05].

Finally, let us return to the discussion on the role of just-infinite groups in the study of growth. Recall that a group $G$ is called just-infinite if it is infinite but every proper quotient is finite. Such groups are on the border between finite groups and infinite groups, and surely they should play an important role in investigations around various gap type conjectures considered in this article. The following statement is an easy application of Zorn's lemma.

Proposition 14.1. Let $G$ be a finitely generated infinite group. Then $G$ has a just-infinite quotient.

Corollary 14.2. Let $\mathcal{P}$ be a group theoretical property preserved under taking quotients. If there is a finitely generated group satisfying the property $\mathcal{P}$ then there is a just-infinite group satisfying this property.

Although the property of a group to have intermediate growth is not preserved when passing to a quotient group (the image may have polynomial growth), by theorems of Gromov [Gro81a] and Rosset [Ros76], if the quotient $G / H$ of a group $G$ of intermediate growth 
is a virtually nilpotent group then $H$ is a finitely generated group of intermediate growth and one may look for a just-infinite quotient of $H$ and iterate this process in order to represent $G$ as a consecutive extension of a chain of groups that are virtually nilpotent or just-infinite groups. This observation is the base of the arguments for statements given by theorems 10.4, $10.3,10.5$ and 14.6 .

The next theorem was derived by the author from a result of J.S. Wilson [Wil71].

Theorem 14.3. ([Gri00b]) The class of just-infinite groups naturally splits into three subclasses:

(B) Algebraically branch just-infinite groups,

(H) Hereditary just-infinite groups, and

(S) near-simple just-infinite groups.

Recall that branch groups were already defined in section 9. The definition of algebraically branch groups can be found in [Gri00a, BGŠ03]. Every geometrically branch group is algebraically branch but not vice versa. The difference between the two versions of the definitions is not large but still there is no complete understanding how much the two classes differ. Not every branch group is just-infinite but every proper quotient of a branch group is virtually abelian. Therefore branch groups are "almost just-infinite" and most of the known finitely generated branch groups are just-infinite.

Definition 14.4. A group $G$ is hereditary just-infinite if it is infinite, residually finite, and every subgroup $H<G$ of finite index is just-infinite.

For instance $\mathbb{Z}, D_{\infty}$, and $P S L(n, \mathbb{Z}), n \geq 3$ (by the result of G.Margulis [Mar91]) are hereditary just-infinite groups.

Definition 14.5. We call a group $G$ near-simple if it contains a subgroup of finite index $H$ which is a direct product

$$
H=P \times P \cdots \times P
$$

of finitely many copies of a simple group $P$.

We already know that there are finitely generated branch groups of intermediate growth (for instance groups $G_{\omega}, \omega \in \Omega_{1}$ ). The question on the existence of non-elementary amenable hereditary just-infinite groups is still open (observe that the only elementary amenable hereditary just-infinite groups are $\mathbb{Z}$ and $\left.D_{\infty}\right)$.

Problem 14. Are there finitely generated hereditary just-infinite groups of intermediate growth?

Problem 15. Are there finitely generated simple groups of intermediate growth?

As it was already mentioned in section 10, we believe that there is a reduction of the Gap Conjecture to the class of just-infinite groups, that is to the classes of (just-infinite) branch groups, hereditary just-infinite groups and simple groups. The corresponding result would hold if the Gap Conjecture holds for residually solvable groups. Using the results of Wilson [Wil], one can prove the following result.

Theorem 14.6. ([Gri12])

(i) If the Gap Conjecture with parameter $1 / 6$ holds for just-infinite groups, then it holds for all groups. 
(ii) If the Gap Conjecture holds for residually polycyclic groups and for just-infinite groups, then it holds for all groups.

Therefore to obtain a complete reduction of the Gap Conjecture to just-infinite groups it is enough to prove it for residually polyciclic groups which is quite plausible. Similar reductions hold for some other gap type conjectures stated in this article.

As was already mentioned in section 10, uncountably many finitely generated simple groups that belong to the class $L E F$ were recently constructed by K. Medynets and the author [GM11]. It may happen that among the subgroups considered in [GM11] (they are commutator subgroups of topological full groups of subshifts of finite type), there are groups of intermediate growth but this has to be checked. On the other hand, it may happen that there are no simple groups of intermediate growth and that there are no hereditary just-infinite groups of intermediate growth at all. In this case the Gap Conjecture would be reduced to the case of branch groups. In [Gri84b, Gri85a] the author proved that growth functions of all p-groups of intermediate growth $\mathcal{G}_{\omega}$ discussed in section 9 satisfy the lower bound $\gamma_{\mathcal{G}_{\omega}}(n) \succeq e^{\sqrt{n}}$, and this was proved by direct computations based on the anti-contracting property given by definition 11.7. This gives some hope that the Gap Conjecture can be proved for the class of branch groups by a similar method.

\section{REFERENCES}

[AAV09] A. Amir, O. Angel, and B. Virag, Amenability of linear-activity automaton groups, 2009, arXiv:0905.2007

[Adi79] S. I. Adian, The Burnside problem and identities in groups, Ergebnisse der Mathematik und ihrer Grenzgebiete [Results in Mathematics and Related Areas], vol. 95, Springer-Verlag, Berlin, 1979. MR 80d:20035

[Adi82] _ _ Random walks on free periodic groups, Izv. Akad. Nauk SSSR Ser. Mat. 46 (1982), no. 6, 1139-1149, 1343. MR 84m:43001

[AK63] V. I. Arnol'd and A. L. Krylov, Uniform distribution of points on a sphere and certain ergodic properties of solutions of linear ordinary differential equations in a complex domain, Dokl. Akad. Nauk SSSR 148 (1963), 9-12. MR 0150374 (27 \#375)

[Ale72] S. V. Alešin, Finite automata and the Burnside problem for periodic groups, Mat. Zametki 11 (1972), 319-328. MR 0301107 (46 \#265)

[And87] Michael T. Anderson, On the fundamental group of nonpositively curved manifolds, Math. Ann. 276 (1987), no. 2, 269-278. MR 870965 (88b:53046)

[Ave70] André Avez, Variétés Riemanniennes sans points focaux, C. R. Acad. Sci. Paris Sér. A-B 270 (1970), A188-A191. MR 0256305 (41 \#961)

[AVŠ57] G. M. Adel'son-Vel'skiŭ and Yu. A. Šrĕ̌der, The Banach mean on groups, Uspehi Mat. Nauk (N.S.) 12 (1957), no. 6(78), 131-136. MR 0094726 (20 \#1238)

[BA10] Laurent Bartholdi and Anna Erschler, Growth of permutational extensions, Invent. Math. 189 (2010), no. 2, 431-455. MR 2947548

[Bab88] I. K. Babenko, Closed geodesics, asymptotic volume and the characteristics of growth of groups, Izv. Akad. Nauk SSSR Ser. Mat. 52 (1988), no. 4, 675-711, 895. MR 966980 (90b:58219)

[Bar98] Laurent Bartholdi, The growth of Grigorchuk's torsion group, Internat. Math. Res. Notices (1998), no. 20, 1049-1054. MR 1656258 (99i:20049)

[Bar01] L Lower bounds on the growth of a group acting on the binary rooted tree, Internat. J. Algebra Comput. 11 (2001), no. 1, 73-88. MR 1818662 (2001m:20044)

[Bar03a] _ Endomorphic presentations of branch groups, J. Algebra 268 (2003), no. 2, 419-443. MR 2009317 (2004h:20044)

[Bar03b] _ A Wilson group of non-uniformly exponential growth, C. R. Math. Acad. Sci. Paris 336 (2003), no. 7, 549-554. MR 1981466 (2004c:20051) 
[BE11] Laurent Bartholdi and Anna Erscler, Groups of given intermediate word growth, 2011, arXiv:1110.3650

[BG00a] Laurent Bartholdi and Rostislav I. Grigorchuk, On the spectrum of Hecke type operators related to some fractal groups, Tr. Mat. Inst. Steklova 231 (2000), no. Din. Sist., Avtom. i Beskon. Gruppy, 5-45. MR 1841750 (2002d:37017)

[BG00b] L Lie methods in growth of groups and groups of finite width, Computational and geometric aspects of modern algebra (Edinburgh, 1998), London Math. Soc. Lecture Note Ser., vol. 275, Cambridge Univ. Press, Cambridge, 2000, pp. 1-27. MR 1776763 (2001h:20046)

[BGN03] Laurent Bartholdi, Rostislav Grigorchuk, and Volodymyr Nekrashevych, From fractal groups to fractal sets, Fractals in Graz 2001, Trends Math., Birkhäuser, Basel, 2003, pp. 25-118. MR 2091700

[BGŠ03] Laurent Bartholdi, Rostislav I. Grigorchuk, and Zoran Šuniḱ, Branch groups, Handbook of algebra, Vol. 3, North-Holland, Amsterdam, 2003, pp. 989-1112. MR 2035113

[BKN10] Laurent Bartholdi, Vadim A. Kaimanovich, and Volodymyr V. Nekrashevych, On amenability of automata groups, Duke Math. J. 154 (2010), no. 3, 575-598. MR 2730578

[BN08] Laurent Bartholdi and Volodymyr V. Nekrashevych, Iterated monodromy groups of quadratic polynomials. I, Groups Geom. Dyn. 2 (2008), no. 3, 309-336.

[BRS06] L. Bartholdi, I. I. Reznykov, and V. I. Sushchansky, The smallest Mealy automaton of intermediate growth, J. Algebra 295 (2006), no. 2, 387-414. MR 2194959 (2006i:68060)

[BV05] Laurent Bartholdi and Bálint Virág, Amenability via random walks, Duke Math. J. 130 (2005), no. 1, 39-56. MR 2176547 (2006h:43001)

[Bas72] H. Bass, The degree of polynomial growth of finitely generated nilpotent groups, Proc. London Math. Soc. (3) 25 (1972), 603-614. MR 0379672 (52 \#577)

[BBES11] M. Bridson, J. Burillo, M. Elder, and Z. Sunic, On groups whose geodesic growth is polynomial, (accepted for publication in the International Journal of Algebra and Computation), 2011.

[BCSDN11] I. Bondarenko, T. Ceccherini-Silberstein, A. Donno, and V. Nekrashevych, On a family of Schreier graphs of intermediate growth associated with a self-similar group, 2011, arXiv:1106.3979

[BdC10] Emmanuel Breuillard and Yves de Cornulier, On conjugacy growth for solvable groups, Illinois J. Math. 54 (2010), no. 1, 389-395. MR 2777001

[BdlHV08] Bachir Bekka, Pierre de la Harpe, and Alain Valette, Kazhdan's property (T), New Mathematical Monographs, vol. 11, Cambridge University Press, Cambridge, 2008. MR 2415834 (2009i:22001)

[BG02a] On parabolic subgroups and Hecke algebras of some fractal groups, Serdica Math. J. 28 (2002), no. 1, 47-90. MR 1899368 (2003c:20027)

[BG02b] Martin R. Bridson and Robert H. Gilman, Context-free languages of sub-exponential growth, J. Comput. System Sci. 64 (2002), no. 2, 308-310. MR 1906807 (2003d:68132)

[BGK $\left.{ }^{+} 08\right]$ Ievgen Bondarenko, Rostislav Grigorchuk, Rostyslav Kravchenko, Yevgen Muntyan, Volodymyr Nekrashevych, Dmytro Savchuk, and Zoran Šunić, Classification of groups generated by 3-state automata over 2-letter alphabet, Algebra Discrete Math. (2008), no. 1, 1-163, arXiv:0803.3555

[Bek04] L. A. Beklaryan, Groups of homeomorphisms of the line and the circle. Topological characteristics and metric invariants, Uspekhi Mat. Nauk 59 (2004), no. 4(358), 3-68. MR 2106645 (2005i:37044)

[Bek08] Levon A. Beklaryan, Groups homeomorphisms: topological characteristics, invariant measures and classifications, Quasigroups Related Systems 16 (2008), no. 2, 155-174. MR 2494874 (2010d:54054)

[Ben83] M. Benson, Growth series of finite extensions of $\mathbb{Z}^{n}$ are rational, Invent. Math. 73 (1983), no. 2, 251-269. MR 714092 (85e:20026)

[BH99] Martin R. Bridson and André Haefliger, Metric spaces of non-positive curvature, Grundlehren der Mathematischen Wissenschaften [Fundamental Principles of Mathematical Sciences], vol. 319, Springer-Verlag, Berlin, 1999. MR 1744486 (2000k:53038)

[BH05] Itai Benjamini and Christopher Hoffman, w-periodic graphs, Electron. J. Combin. 12 (2005), Research Paper 46, 12 pp. (electronic). MR 2176522 (2006f:05151)

[BM88] Gilbert Baumslag and Charles F. Miller, III, Some odd finitely presented groups, Bull. London Math. Soc. 20 (1988), no. 3, 239-244. MR 931184 (89e:20059) 
[BM07] B. Bajorska and O. Macedońska, A note on groups of intermediate growth, Comm. Algebra 35 (2007), no. 12, 4112-4115. MR 2372323 (2008j:20082)

[BOERT96] Hyman Bass, Maria Victoria Otero-Espinar, Daniel Rockmore, and Charles Tresser, Cyclic renormalization and automorphism groups of rooted trees, Lecture Notes in Mathematics, vol. 1621, Springer-Verlag, Berlin, 1996. MR 1392694 (97k:58058)

[Bog] N.N. Bogolyubov, On some ergodic properties of continious groups of transformations, Nauk. Zap. Kiïv Derzh. Univ. im. T.G.Shevchenka, v. IV, (1939) no. 5, 45-52.

[Bon07] I. Bondarenko, Groups generated by bounded automata and their Schreier graphs, (Dissertation), 2007.

[Bon11]_ Growth of Schreier graphs of automaton groups, 2011, arXiv:1101.3200

[BP06] Kai-Uwe Bux and Rodrigo Pérez, On the growth of iterated monodromy groups, Topological and asymptotic aspects of group theory, Contemp. Math., vol. 394, Amer. Math. Soc., Providence, RI, 2006, pp. 61-76. MR 2216706 (2006m:20062), arXiv:math.GR/0405456.

[BPS09] A. Bendikov, C. Pittet, and R. Sauer, Spectral distribution and $l^{2}$-isoperimetric profile of Laplace operators on groups, 2009, arXiv:0901.0271

[BGH13] M. Benli, R. Grigorchuk and P. de la Harpe, Amenable groups without finitely presented covers. to appear in Bull. Math. Sci. (2013).

[Bre07] Emmanuel Breuillard, On uniform exponential growth for solvable groups, Pure Appl. Math. Q. 3 (2007), no. 4, part 1, 949-967. MR 2402591 (2009d:20073)

[Bri11] J. Brieussel, Growth behaviors in the range $e^{r^{\alpha}}, 2011$, arXiv:1107.1632

[BS07] Ievgen V. Bondarenko and Dmytro M. Savchuk, On Sushchansky p-groups, Algebra Discrete Math. (2007), no. 2, 22-42. MR 2364061 (2008i:20049)

[Can80] J. Cannon, The growth of the closed surface groups and compact hyperbolic Coxeter groups, (preprint), 1980.

[Can84] James W. Cannon, The combinatorial structure of cocompact discrete hyperbolic groups, Geom. Dedicata 16 (1984), no. 2, 123-148. MR 758901 (86j:20032)

[CDP90a] M. Coornaert, T. Delzant, and A. Papadopoulos, Géométrie et théorie des groupes, Lecture Notes in Mathematics, vol. 1441, Springer-Verlag, Berlin, 1990, Les groupes hyperboliques de Gromov. [Gromov hyperbolic groups], With an English summary. MR 1075994 (92f:57003)

[Che78] Su Shing Chen, On the fundamental group of a compact negatively curved manifold, Proc. Amer. Math. Soc. 71 (1978), no. 1, 119-122. MR 0514740 (58 \#24117)

[Cho80] Ching Chou, Elementary amenable groups, Illinois J. Math. 24 (1980), no. 3, 396-407. MR 573475 (81h:43004)

[CK02] M. Coornaert and G. Knieper, Growth of conjugacy classes in Gromov hyperbolic groups, Geom. Funct. Anal. 12 (2002), no. 3, 464-478. MR 1924369 (2003f:20071)

[CM97] Tobias H. Colding and William P. Minicozzi, II, Harmonic functions on manifolds, Ann. of Math. (2) 146 (1997), no. 3, 725-747. MR 1491451 (98m:53052)

[CSC93] Thierry Coulhon and Laurent Saloff-Coste, Isopérimétrie pour les groupes et les variétés, Rev. Mat. Iberoamericana 9 (1993), no. 2, 293-314. MR 1232845 (94g:58263)

[CSC10] Tullio Ceccherini-Silberstein and Michel Coornaert, Cellular automata and groups, Springer Monographs in Mathematics, Springer-Verlag, Berlin, 2010. MR 2683112

[CSG97] Tullio G. Ceccherini-Silberstein and Rostislav I. Grigorchuk, Amenability and growth of onerelator groups, Enseign. Math. (2) 43 (1997), no. 3-4, 337-354. MR 1489891 (99b:20057)

[CSMS01] Tullio Ceccherini-Silberstein, Antonio Machì, and Fabio Scarabotti, The Grigorchuk group of intermediate growth, Rend. Circ. Mat. Palermo (2) 50 (2001), no. 1, 67-102. MR 1825671 (2002a:20044)

[CSW02] Tullio Ceccherini-Silberstein and Wolfgang Woess, Growth and ergodicity of context-free languages, Trans. Amer. Math. Soc. 354 (2002), no. 11, 4597-4625. MR 1926891 (2003g:68067)

[CSW03]__ Growth-sensitivity of context-free languages, Theoret. Comput. Sci. 307 (2003), no. 1, 103-116, Words. MR 2022843 (2005h:68066)

[Day57] Mahlon M. Day, Amenable semigroups, Illinois J. Math. 1 (1957), 509-544. MR 19,1067c

[Dix60] Jacques Dixmier, Opérateurs de rang fini dans les représentations unitaires, Inst. Hautes Études Sci. Publ. Math. (1960), no. 6, 13-25. MR 0136684 (25 \#149) 
[dlH73] Pierre de la Harpe, Moyennabilité de quelques groupes topologiques de dimension infinie, C. R. Acad. Sci. Paris Sér. A-B 277 (1973), A1037-A1040. MR 0333060 (48 \#11385)

[dlH00] , Topics in geometric group theory, Chicago Lectures in Mathematics, University of Chicago Press, Chicago, IL, 2000. MR 1786869 (2001i:20081)

[dlH02] Uniform growth in groups of exponential growth, Proceedings of the Conference on Geometric and Combinatorial Group Theory, Part II (Haifa, 2000), vol. 95, 2002, pp. 1-17. MR 1950882 (2003k:20031)

[dlHGCS99] P. de la Harpe, R. I. Grigorchuk, and T. Chekerini-Sil'berstaĭn, Amenability and paradoxical decompositions for pseudogroups and discrete metric spaces, Tr. Mat. Inst. Steklova 224 (1999), no. Algebra. Topol. Differ. Uravn. i ikh Prilozh., 68-111. MR 1721355 (2001h:43001)

[DM61] E. B. Dynkin and M. B. Maljutov, Random walk on groups with a finite number of generators, Dokl. Akad. Nauk SSSR 137 (1961), 1042-1045. MR 0131904 (24 \#A1751)

[Dye59] H. A. Dye, On groups of measure preserving transformation. I, Amer. J. Math. 81 (1959), 119-159. MR 0131516 (24 \#A1366)

[Dye63] _ _ On groups of measure preserving transformations. II, Amer. J. Math. 85 (1963), 551576. MR 0158048 (28 \#1275)

[Ebe73] Patrick Eberlein, Some properties of the fundamental group of a Fuchsian manifold, Invent. Math. 19 (1973), 5-13. MR 0400250 (53 \#4085)

[Efr53] V. A. Efremovič, The proximity geometry of Riemannien manifolds., Uspekhi Math. Nauk. 8 (1953), 189.

[EK10] Anna Erschler and Anders Karlsson, Homomorphisms to $\mathbb{R}$ constructed from random walks, Ann. Inst. Fourier (Grenoble) 60 (2010), no. 6, 2095-2113. MR 2791651

[EMO05] Alex Eskin, Shahar Mozes, and Hee Oh, On uniform exponential growth for linear groups, Invent. Math. 160 (2005), no. 1, 1-30. MR 2129706 (2006a:20081)

[Ers04a] Anna Erschler, Boundary behavior for groups of subexponential growth, Annals of Math. 160 (2004), no. 3, 1183-1210.

[Ers04b] - Not residually finite groups of intermediate growth, commensurability and nongeometricity, J. Algebra 272 (2004), no. 1, 154-172. MR 2029029 (2004j:20066)

[Ers05a] Critical constants for recurrence of random walks on G-spaces, Ann. Inst. Fourier (Grenoble) $5 \mathbf{5}$ (2005), no. 2, 493-509. MR 2147898 (2006c:20085)

[Ėrs05b] A. G. Ėrshler, On the degrees of growth of finitely generated groups, Funktsional. Anal. i Prilozhen. 39 (2005), no. 4, 86-89. MR 2197519 (2006k:20056)

[Ers06] Anna Erschler, Piecewise automatic groups, Duke Math. J. 134 (2006), no. 3, 591-613. MR 2254627 (2007k:20086)

[Ers10] _ Poisson-Furstenberg boundaries, large-scale geometry and growth of groups, Proc. ICM, Vol III. Hindustan Book Agency, New Dehli, 2010, 681-704. MR 2827814 (2012h:60016)

[Føl57] Erling Følner, Note on groups with and without full Banach mean value, Math. Scand. 5 (1957), 5-11. MR 0094725 (20 \#1237)

[FT95] Michael H. Freedman and Peter Teichner, 4-manifold topology. I. Subexponential groups, Invent. Math. 122 (1995), no. 3, 509-529. MR 1359602 (96k:57015)

[GH90] Étienne Ghys and André Haefliger, Groupes de torsion, Sur les groupes hyperboliques d'après Mikhael Gromov (Bern, 1988), Progr. Math., vol. 83, Birkhäuser Boston, Boston, MA, 1990, pp. 215-226. MR 1086660

[Ghy01] Étienne Ghys, Groups acting on the circle, Enseign. Math. (2) 47 (2001), no. 3-4, 329-407. MR 1876932 (2003a:37032)

[Gil05] Robert H. Gilman, Formal languages and their application to combinatorial group theory, Groups, languages, algorithms, Contemp. Math., vol. 378, Amer. Math. Soc., Providence, RI, 2005, pp. 1-36. MR 2159313 (2006g:68142)

[Glu61] V. M. Gluškov, Abstract theory of automata, Uspehi Mat. Nauk 16 (1961), no. 5 (101), 3-62. MR 0138529 (25 \#1976)

[Gol64] E. S. Golod, On nil-algebras and finitely approximable p-groups, Izv. Akad. Nauk SSSR Ser. Mat. 28 (1964), 273-276. MR 0161878 (28 \#5082)

[Gre69] Frederick P. Greenleaf, Invariant means on topological groups and their applications, Van Nostrand Mathematical Studies, No. 16, Van Nostrand Reinhold Co., New York, 1969. 
[GdlH97] R. Grigorchuk and P. de la Harpe, On problems related to growth, entropy, and spectrum in group theory, J. Dynam. Control Systems 3 (1997), no. 1, 51-89. MR 1436550 (98d:20039)

[GdlH01] _ One-relator groups of exponential growth have uniformly exponential growth, Mat. Zametki 69 (2001), no. 4, 628-630. MR 1846003 (2002b:20041)

[GM93] R. I. Grigorchuk and A. Machi, On a group of intermediate growth that acts on a line by homeomorphisms, Mat. Zametki 53 (1993), no. 2, 46-63. MR 1220809 (94c:20008)

[GM99] R. I. Grigorchuk and A. Machì, An example of an indexed language of intermediate growth, Theoret. Comput. Sci. 215 (1999), no. 1-2, 325-327. MR 1678812 (99k:68092)

[GM11] R. Gigorchuk and K. Medynets, Topological full groups are locally embeddable into finite groups, 2011, arXiv:1105.0719

[GN97] Rostislav Grigorchuk and Tatiana Nagnibeda, Complete growth functions of hyperbolic groups, Invent. Math. 130 (1997), no. 1, 159-188. MR 1471889 (98i:20038)

[GN05] R. Grigorchuk and V. Nekrashevych, Amenable actions of nonamenable groups, Zap. Nauchn. Sem. S.-Peterburg. Otdel. Mat. Inst. Steklov. (POMI) 326 (2005), no. Teor. Predst. Din. Sist. Komb. i Algoritm. Metody. 13, 85-96, 281. MR 2183217 (2006j:43005)

[GN07] Rostislav Grigorchuk and Volodymyr Nekrashevych, Self-similar groups, operator algebras and Schur complement, J. Modern Dyn. 1 (2007), no. 3, 323-370.

[GNS00] R. I. Grigorchuk, V. V. Nekrashevich, and V. I. Sushchanskǐr, Automata, dynamical systems, and infinite groups, Tr. Mat. Inst. Steklova 231 (2000), no. Din. Sist., Avtom. i Beskon. Gruppy, 134-214. MR 1841755 (2002m:37016)

[GP08] Rostislav Grigorchuk and Igor Pak, Groups of intermediate growth: an introduction, Enseign. Math. (2) 54 (2008), no. 3-4, 251-272. MR 2478087 (2009k:20101)

[Gri] Rostislav Grigorchuk, Some Topics in the Dynamics of Group Actions on Rooted Trees, Proceedings of the Steklov Institute of Mathematics.

[Gri79] R. I. Grigorčuk, Invariant measures on homogeneous spaces, Ukrain. Mat. Zh. 31 (1979), no. 5, 490-497, 618. MR 552478 (81k:60073)

[Gri80a] R. I. Grigorchuk, Symmetrical random walks on discrete groups, Multicomponent random systems, Adv. Probab. Related Topics, vol. 6, Dekker, New York, 1980, pp. 285-325. MR 599539 (83k:60016)

[Gri80b] R. I. Grigorčuk, On Burnside's problem on periodic groups, Funktsional. Anal. i Prilozhen. 14 (1980), no. 1, 53-54. MR 565099 (81m:20045)

[Gri83] R. I. Grigorchuk, On the Milnor problem of group growth, Dokl. Akad. Nauk SSSR 271 (1983), no. 1, 30-33. MR 712546 (85g:20042)

[Gri84a] Construction of p-groups of intermediate growth that have a continuum of factor-groups, Algebra i Logika 23 (1984), no. 4, 383-394, 478. MR 781246 (86h:20058)

[Gri84b] Degrees of growth of finitely generated groups and the theory of invariant means, Izv. Akad. Nauk SSSR Ser. Mat. 48 (1984), no. 5, 939-985. MR 764305 (86h:20041)

[Gri85a] Degrees of growth of p-groups and torsion-free groups, Mat. Sb. (N.S.) 126(168) (1985), no. 2, 194-214, 286. MR 784354 (86m:20046)

[Gri85b] R.I. Grigorchuk, Groups with intermediate growth function and their applications, Habilitation, Steklov Institute of Mathematics, 1985.

[Gri88] R. I. Grigorchuk, Semigroups with cancellations of polynomial growth, Mat. Zametki 43 (1988), no. 3, 305-319, 428. MR 941053 (89f:20065)

[Gri89a] On the Hilbert-Poincaré series of graded algebras that are associated with groups, Mat. Sb. 180 (1989), no. 2, 207-225, 304. MR 993455 (90j:20063)

[Gri89b] - Topological and metric types of surfaces that regularly cover a closed surface, Izv. Akad. Nauk SSSR Ser. Mat. 53 (1989), no. 3, 498-536, 671. MR 1013710 (90j:57002)

[Gri91] Rostislav I. Grigorchuk, On growth in group theory, Proceedings of the International Congress of Mathematicians, Vol. I, II (Kyoto, 1990) (Tokyo), Math. Soc. Japan, 1991, pp. 325-338. MR 1159221 (93e:20001)

[Gri98] R. I. Grigorchuk, An example of a finitely presented amenable group that does not belong to the class EG, Mat. Sb. 189 (1998), no. 1, 79-100. MR 1616436 (99b:20055)

[Gri00a] _ Branch groups, Mat. Zametki 67 (2000), no. 6, 852-858. MR 1820639 (2001i:20057) 
[Gri00b] _ Just infinite branch groups, New horizons in pro-p groups, Progr. Math., vol. 184, Birkhäuser Boston, Boston, MA, 2000, pp. 121-179. MR 1765119 (2002f:20044)

[Gri05] Rostislav Grigorchuk, Solved and unsolved problems around one group, Infinite groups: geometric, combinatorial and dynamical aspects, Progr. Math., vol. 248, Birkhäuser, Basel, 2005, pp. 117-218. MR 2195454

[Gri12] Rostislav Grigorchuk, On the gap conjecture concerning group growth, 2012, arXiv:1202.6044

[Gro81a] Mikhael Gromov, Groups of polynomial growth and expanding maps, Inst. Hautes Études Sci. Publ. Math. (1981), no. 53, 53-73. MR 623534 (83b:53041)

[Gro81b] _ Structures métriques pour les variétés riemanniennes, Textes Mathématiques [Mathematical Texts], vol. 1, CEDIC, Paris, 1981, Edited by J. Lafontaine and P. Pansu. MR 682063 (85e:53051)

[Gro87] M. Gromov, Hyperbolic groups, Essays in group theory, Math. Sci. Res. Inst. Publ., vol. 8, Springer, New York, 1987, pp. 75-263. MR 919829 (89e:20070)

[Gro93] - Asymptotic invariants of infinite groups, Geometric group theory, Vol. 2 (Sussex, 1991), London Math. Soc. Lecture Note Ser., vol. 182, Cambridge Univ. Press, Cambridge, 1993, pp. 1295. MR 1253544 (95m:20041)

[Gro08] Misha Gromov, Entropy and isoperimetry for linear and non-linear group actions, Groups Geom. Dyn. 2 (2008), no. 4, 499-593. MR 2442946 (2010h:37011)

[GŠ64] E. S. Golod and I. R. Šafarevič, On the class field tower, Izv. Akad. Nauk SSSR Ser. Mat. 28 (1964), 261-272. MR 0161852 (28 \#5056)

[GS83] Narain Gupta and Saïd Sidki, On the Burnside problem for periodic groups, Math. Z. 182 (1983), no. 3, 385-388. MR 696534 (84g:20075)

[GŠ06] Rostislav Grigorchuk and Zoran Šuniḱ, Asymptotic aspects of Schreier graphs and Hanoi Towers groups, C. R. Math. Acad. Sci. Paris 342 (2006), no. 8, 545-550. MR 2217913

[GSS88] F. J. Grunewald, D. Segal, and G. C. Smith, Subgroups of finite index in nilpotent groups, Invent. Math. 93 (1988), no. 1, 185-223. MR 943928 (89m:11084)

[Gui70] Yves Guivarc'h, Groupes de Lie à croissance polynomiale, C. R. Acad. Sci. Paris Sér. A-B 271 (1970), A237-A239. MR 0272943 (42 \#7824)

[Gui71] $\quad$ Groupes de Lie à croissance polynomiale, C. R. Acad. Sci. Paris Sér. A-B 272 (1971), A1695-A1696. MR 0302819 (46 \#1962)

[Gui73] _ Croissance polynomiale et périodes des fonctions harmoniques, Bull. Soc. Math. France 101 (1973), 333-379. MR 0369608 (51 \#5841)

[Gui80] Y. Guivarc'h, Sur la loi des grands nombres et le rayon spectral d'une marche aléatoire, Conference on Random Walks (Kleebach, 1979) (French), Astérisque, vol. 74, Soc. Math. France, Paris, 1980, pp. 47-98, 3. MR 588157 (82g:60016)

[GŻ02] Rostislav I. Grigorchuk and Andrzej Żuk, On a torsion-free weakly branch group defined by a three state automaton, Internat. J. Algebra Comput. 12 (2002), no. 1-2, 223-246. MR 1902367 (2003c:20048)

[HO11] M. Hull and D. Osin, Conjugacy growth of finitely generated groups, 2011, arXiv:1107.1826

[Hop48] Eberhard Hopf, Closed surfaces without conjugate points, Proc. Nat. Acad. Sci. U. S. A. 34 (1948), 47-51. MR 0023591 (9,378d)

[HR79] Edwin Hewitt and Kenneth A. Ross, Abstract harmonic analysis. Vol. I, second ed., Grundlehren der Mathematischen Wissenschaften [Fundamental Principles of Mathematical Sciences], vol. 115, Springer-Verlag, Berlin, 1979, Structure of topological groups, integration theory, group representations. MR 551496 (81k:43001)

[Inc01] Roberto Incitti, The growth function of context-free languages, Theoret. Comput. Sci. 255 (2001), no. 1-2, 601-605. MR 1819093 (2001m:68098)

[Jon74] J. M. Tyrer Jones, Direct products and the Hopf property, J. Austral. Math. Soc. 17 (1974), 174-196, Collection of articles dedicated to the memory of Hanna Neumann, VI. MR 0349855 (50 \#2348)

[JM12] Kate Juschenko and Nicolas Monod, Cantor systems, piecewise translations and simple amenable groups, 2012. arXiv:1204.2132

[Kai05] Vadim A. Kaimanovich, "Münchhausen trick" and amenability of self-similar groups, Internat. J. Algebra Comput. 15 (2005), no. 5-6, 907-937. MR 2197814 
[KAP85] V. B. Kudryavtsev, S. V. Aleshin, and A. S. Podkolzin, Vvedenie v teoriyu avtomatov, "Nauka", Moscow, 1985. MR 837853 (87k:68112)

[Kes59a] Harry Kesten, Full Banach mean values on countable groups, Math. Scand. 7 (1959), 146-156. MR 0112053 (22 \#2911)

[Kes59b] _ Symmetric random walks on groups, Trans. Amer. Math. Soc. 92 (1959), 336-354. MR 0109367 (22 \#253)

[Kir67] A. A. Kirillov, Dynamical systems, factors and group representations, Uspehi Mat. Nauk 22 (1967), no. 5 (137), 67-80. MR 0217256 (36 \#347)

[KK74] Ali Ivanovič Kokorin and Valerī̄ Matveevič Kopytov, Fully ordered groups, Halsted Press [John Wiley \& Sons], New York-Toronto, Ont., 1974, Translated from the Russian by D. Louvish. MR 0364051 (51 \#306)

[KL00] Günter R. Krause and Thomas H. Lenagan, Growth of algebras and Gelfand-Kirillov dimension, revised ed., Graduate Studies in Mathematics, vol. 22, American Mathematical Society, Providence, RI, 2000. MR 1721834 (2000j:16035)

[KL07] Anders Karlsson and François Ledrappier, Linear drift and Poisson boundary for random walks, Pure Appl. Math. Q. 3 (2007), no. 4, Special Issue: In honor of Grigory Margulis. Part 1, 10271036. MR 2402595 (2009d:60133)

[Kla81a] D. A. Klarner, Mathematical crystal growth. I, Discrete Appl. Math. 3 (1981), no. 1, 47-52. MR 604265 (82e:05016)

[Kla81b] _ Mathematical crystal growth. II, Discrete Appl. Math. 3 (1981), no. 2, 113-117. MR 607910 (83a:05018)

[Kle10] Bruce Kleiner, A new proof of Gromov's theorem on groups of polynomial growth, J. Amer. Math. Soc. 23 (2010), no. 3, 815-829. MR 2629989

[Kou98] Malik Koubi, Croissance uniforme dans les groupes hyperboliques, Ann. Inst. Fourier (Grenoble) 48 (1998), no. 5, 1441-1453. MR 1662255 (99m:20080)

[KP11] M. Kassabov and I. Pak, Groups of oscillating intermediate growth, Annals Math, 177 (2013), no. 3, 1113-1145. arXiv:1108.0268

[Kra53] Hans Ulrich Krause, Gruppenstruktur und Gruppenbild, Thesis, Eidgenössische Technische Hochschule, Zürich, 1953. MR 0056599 (15,99b)

[KV83] V. A. Kaŭmanovich and A. M. Vershik, Random walks on discrete groups: boundary and entropy, Ann. Probab. 11 (1983), no. 3, 457-490. MR 704539 (85d:60024)

[Laz65] Michel Lazard, Groupes analytiques p-adiques, Inst. Hautes Études Sci. Publ. Math. (1965), no. 26, 389-603. MR 0209286 (35 \#188)

[Leo01] Yu. G. Leonov, On a lower bound for the growth of a 3-generator 2-group, Mat. Sb. 192 (2001), no. 11, 77-92. MR 1886371 (2003a:20050)

[Lia96] F. Liardet, Croissance dans les groupes virtuellement abéliens, Thèse, Uuniversity of Geneève, 1996.

[LM91] Alexander Lubotzky and Avinoam Mann, On groups of polynomial subgroup growth, Invent. Math. 104 (1991), no. 3, 521-533. MR 1106747 (92d:20038)

[LMR95] P. Longobardi, M. Maj, and A. H. Rhemtulla, Groups with no free subsemigroups, Trans. Amer. Math. Soc. 347 (1995), no. 4, 1419-1427. MR 1277124 (95g:20043)

[LMS93] Alexander Lubotzky, Avinoam Mann, and Dan Segal, Finitely generated groups of polynomial subgroup growth, Israel J. Math. 82 (1993), no. 1-3, 363-371. MR 1239055 (95b:20051)

[LS77] Roger C. Lyndon and Paul E. Schupp, Combinatorial group theory, Springer-Verlag, Berlin, 1977, Ergebnisse der Mathematik und ihrer Grenzgebiete, Band 89. MR 0577064 (58 \#28182)

[LS03] Alexander Lubotzky and Dan Segal, Subgroup growth, Progress in Mathematics, vol. 212, Birkhäuser Verlag, Basel, 2003. MR 1978431 (2004k:20055)

[Lub94] Alexander Lubotzky, Discrete groups, expanding graphs and invariant measures, Progress in Mathematics, vol. 125, Birkhäuser Verlag, Basel, 1994, With an appendix by Jonathan D. Rogawski. MR 1308046 (96g:22018)

[Lys85] I. G. Lysënok, A set of defining relations for the Grigorchuk group, Mat. Zametki 38 (1985), no. 4, 503-516, 634. MR 819415 (87g:20062)

[Mal53] A. I. Mal'cev, Nilpotent semigroups, Ivanov. Gos. Ped. Inst. Uč. Zap. Fiz.-Mat. Nauki 4 (1953), 107-111. MR 0075959 (17,825d) 
[Man07] Avinoam Mann, Growth conditions in infinitely generated groups, Groups Geom. Dyn. 1 (2007), no. 4, 613-622. MR 2357485 (2008k:20101)

[Mang10] Johanna Mangahas, Uniform exponential growth of subgroups of the mapping class group, Geom. Funct. Anal. 19 (2010), no. 5, 1468-1480. MR 2585580 (2011d:57002)

[Man12] Avinoam Mann, How groups grow, Cambridge Univ. Press, 2012, London Math. Soc. Lecture Notes, v. 395.

[Mar66] G. A. Margulis, Positive harmonic functions on nilpotent groups, Soviet Math. Dokl. 7 (1966), 241-244. MR 0222217 (36 \#5269)

[Mar91] _ Discrete subgroups of semisimple Lie groups, Ergebnisse der Mathematik und ihrer Grenzgebiete (3) [Results in Mathematics and Related Areas (3)], vol. 17, Springer-Verlag, Berlin, 1991. MR 1090825 (92h:22021)

[Mat06] Hiroki Matui, Some remarks on topological full groups of Cantor minimal systems, Internat. J. Math. 17 (2006), no. 2, 231-251. MR 2205435 (2007f:37011)

[Mat11]_ Some remarks on topological full groups of Cantor minimal systems ii, 2011, arXiv:1111.3134

[Mil68a] J. Milnor, A note on curvature and fundamental group, J. Differential Geometry 2 (1968), 1-7. MR 0232311 (38 \#636)

[Mil68b] _ Problem 5603, Amer. Math. Monthly 75 (1968), 685-686.

[Mil68c] John Milnor, Growth of finitely generated solvable groups, J. Differential Geometry 2 (1968), 447-449. MR 0244899 (39 \#6212)

[MM93] Antonio Machì and Filippo Mignosi, Garden of Eden configurations for cellular automata on Cayley graphs of groups, SIAM J. Discrete Math. 6 (1993), no. 1, 44-56. MR 1201989 (95a:68084)

[Mor06] Dave Witte Morris, Amenable groups that act on the line, Algebr. Geom. Topol. 6 (2006), 2509-2518. MR 2286034 (2008c:20078)

[MP01a] Roman Muchnik and Igor Pak, On growth of Grigorchuk groups, Internat. J. Algebra Comput. 11 (2001), no. 1, 1-17. MR 1818659 (2002e:20066)

[MP01b] _ Percolation on Grigorchuk groups, Comm. Algebra 29 (2001), no. 2, 661-671. MR 1841989 (2002e:82033)

[Muc05] Roman Muchnik, Amenability of universal 2-Grigorchuk group, arXiv:0802.2837, 2005.

[MZ74] Deane Montgomery and Leo Zippin, Topological transformation groups, Robert E. Krieger Publishing Co., Huntington, N.Y., 1974, Reprint of the 1955 original. MR 0379739 (52 \#644)

[NA68a] P. S. Novikov and S. I. Adjan, Infinite periodic groups. I, Izv. Akad. Nauk SSSR Ser. Mat. 32 (1968), 212-244. MR 0240178 (39 \#1532a)

[NA68b] _ Infinite periodic groups. II, Izv. Akad. Nauk SSSR Ser. Mat. 32 (1968), 251-524. MR 0240179 (39 \#1532b)

[NA68c] _ Infinite periodic groups. II, Izv. Akad. Nauk SSSR Ser. Mat. 32 (1968), 251-524. MR 0240179 (39 \#1532b)

[NA68d] _ Infinite periodic groups. III, Izv. Akad. Nauk SSSR Ser. Mat. 32 (1968), 709-731. MR 0240180 (39 \#1532c)

[Nav08] Andrés Navas, Growth of groups and diffeomorphisms of the interval, Geom. Funct. Anal. 18 (2008), no. 3, 988-1028. MR 2439001 (2010c:37086)

[Nav11] Groups of circle diffeomorphisms, spanish ed., Chicago Lectures in Mathematics, University of Chicago Press, Chicago, IL, 2011. MR 2809110

[Nek05] Volodymyr Nekrashevych, Self-similar groups, Mathematical Surveys and Monographs, vol. 117, American Mathematical Society, Providence, RI, 2005. MR 2162164

[Nek07] _ A minimal Cantor set in the space of 3-generated groups, Geom. Dedicata 124 (2007), 153-190. MR 2318543 (2008d:20075)

[Nek10] A Aroup of non-uniform exponential growth locally isomorphic to $\operatorname{IMG}\left(z^{2}+i\right)$, Trans. Amer. Math. Soc. 362 (2010), no. 1, 389-398. MR 2550156 (2010m:20067)

[NS95] Walter D. Neumann and Michael Shapiro, Automatic structures, rational growth, and geometrically finite hyperbolic groups, Invent. Math. 120 (1995), no. 2, 259-287. MR 1329042 (96c:20066) 
[NY12] Piotor W. Nowak and Guoliang Yu, Large scale geometry, EMS Textbooks in Mathematics, European Mathematical Scociety, Zürich, 2012. MR 2986138

[Ol'80] A. Ju. Ol'šanskiı̌, On the question of the existence of an invariant mean on a group, Uspekhi Mat. Nauk 35 (1980), no. 4(214), 199-200. MR 586204 (82b:43002)

[OS02] Alexander Yu. Ol'shanskii and Mark V. Sapir, Non-amenable finitely presented torsion-bycyclic groups, Publ. Math. Inst. Hautes Études Sci. (2002), no. 96, 43-169 (2003). MR 1985031 (2004f:20061)

[Osi02] D. V. Osin, Elementary classes of groups, Mat. Zametki 72 (2002), no. 1, 84-93. MR 1942584 (2003h:20062)

[Osi03] _ The entropy of solvable groups, Ergodic Theory Dynam. Systems 23 (2003), no. 3, 907-918. MR 1992670 (2004f:20065)

[Osi04] _ Algebraic entropy of elementary amenable groups, Geom. Dedicata 107 (2004), 133151. MR 2110759 (2006b:37010)

[Pan83] Pierre Pansu, Croissance des boules et des géodésiques fermées dans les nilvariétés, Ergodic Theory Dynam. Systems 3 (1983), no. 3, 415-445. MR 741395 (85m:53040)

[Par91] Luis Paris, Growth series of Coxeter groups, Group theory from a geometrical viewpoint (Trieste, 1990), World Sci. Publ., River Edge, NJ, 1991, pp. 302-310. MR 1170370 (93g:20081)

[PSZ10] Victor M. Petrogradsky, Ivan P. Shestakov, and Efim Zelmanov, Nil graded self-similar algebras, Groups Geom. Dyn. 4 (2010), no. 4, 873-900. MR 2727670 (2011i:17028)

[Rob96] Derek J. S. Robinson, A course in the theory of groups, second ed., Graduate Texts in Mathematics, vol. 80, Springer-Verlag, New York, 1996. MR 1357169 (96f:20001)

[Roe03] John Roe, Lectures on coarse geometry, University Lecture Series, vol. 31, American Mathematical Society, Providence, RI, 2003. MR 2007488 (2004g:53050)

[Ros74] Joseph Max Rosenblatt, Invariant measures and growth conditions, Trans. Amer. Math. Soc. 193 (1974), 33-53. MR 0342955 (49 \#7699)

[Ros76] Shmuel Rosset, A property of groups of non-exponential growth, Proc. Amer. Math. Soc. 54 (1976), 24-26. MR 0387420 (52 \#8263)

[Sai10] Kyoji Saito, Limit elements in the configuration algebra for a cancellative monoid, Publ. Res. Inst. Math. Sci. 46 (2010), no. 1, 37-113. MR 2662615 (2011i:05095)

[Sai11] Kyoji Saito, Opposite power series, European J. Combin. 33 (2012), no. 7, 1653-1671. MR 2923475

[Shn04] L. M. Shneerson, On semigroups of intermediate growth, Comm. Algebra 32 (2004), no. 5, 1793-1803. MR 2099701 (2005h:20131)

[Shn05] _ Types of growth and identities of semigroups, Internat. J. Algebra Comput. 15 (2005), no. 5-6, 1189-1204. MR 2197827 (2006j:20086)

[Shu70] Michael Shub, Expanding maps, Global Analysis (Proc. Sympos. Pure Math., Vol. XIV, Berkeley, Calif., 1968), Amer. Math. Soc., Providence, R.I., 1970, pp. 273-276. MR 0266251 (42 \#1158)

[Smi64] D. M. Smirnov, Generalized soluble groups and their group rings, Dokl. Akad. Nauk SSSR 155 (1964), 535-537. MR 0163961 (29 \#1260)

[ST10] Yehuda Shalom and Terence Tao, A finitary version of Gromov's polynomial growth theorem, Geom. Funct. Anal. 20 (2010), no. 6, 1502-1547. MR 2739001

[Sto96] Michael Stoll, Rational and transcendental growth series for the higher Heisenberg groups, Invent. Math. 126 (1996), no. 1, 85-109. MR 1408557 (98d:20033)

[Sus79] V. I. Sushchansky, Periodic permutation p-groups and the unrestricted Burnside problem, DAN SSSR. 247 (1979), no. 3, 557-562, (in Russian).

[SW02] Mark Sapir and Daniel T. Wise, Ascending HNN extensions of residually finite groups can be non-Hopfian and can have very few finite quotients, J. Pure Appl. Algebra 166 (2002), no. 1-2, 191-202. MR 1868545 (2002i:20038)

[Tit72] J. Tits, Free subgroups in linear groups, J. Algebra 20 (1972), 250-270. MR 0286898 (44 \#4105)

[Tit81] Jacques Tits, Groupes à croissance polynomiale (d'après M. Gromov et al.), Bourbaki Seminar, Vol. 1980/81, Lecture Notes in Math., vol. 901, Springer, Berlin, 1981, pp. 176-188. MR 647496 (83i:53065) 
[Tro80] V. I. Trofimov, The growth functions of finitely generated semigroups, Semigroup Forum 21 (1980), no. 4, 351-360. MR 597500 (82g:20094)

[Tro84] ㄴ. Graphs with polynomial growth, Mat. Sb. (N.S.) 123(165) (1984), no. 3, $407-421$. MR 735714 (85m:05041)

[Var91] Nicholas Th. Varopoulos, Analysis and geometry on groups, Proceedings of the International Congress of Mathematicians, Vol. I, II (Kyoto, 1990) (Tokyo), Math. Soc. Japan, 1991, pp. 951957. MR 1159280 (93k:22006)

[vdDW84] L. van den Dries and A. J. Wilkie, Gromov's theorem on groups of polynomial growth and elementary logic, J. Algebra 89 (1984), no. 2, 349-374. MR 751150 (85k:20101)

[vN29] John von Neumann, Zurr allgemeinen theorie des masses, Fund.Math. 13 (1929), 73-116.

[Š55] A.S. Švarc, A volume invariant of covering, Dokl. Akad. Nauj SSSR (N.S.), 105 (1955), 32-34. MR $0075634(17,781 \mathrm{~d})$

[VSCC92] N. Th. Varopoulos, L. Saloff-Coste, and T. Coulhon, Analysis and geometry on groups, Cambridge Tracts in Mathematics, vol. 100, Cambridge University Press, Cambridge, 1992. MR 1218884 (95f:43008)

[Wag93] Stan Wagon, The Banach-Tarski paradox, Cambridge University Press, Cambridge, 1993, With a foreword by Jan Mycielski, Corrected reprint of the 1985 original. MR 1251963 (94g:04005)

[Wil] J. Wilson, The gap in the growth of residually soluble groups, Bull. Lond. Math. Soc. 43 (2011), no. 3, 576-582. MR 2820146 (2012f:20105)

[Wil71] J. S. Wilson, Groups with every proper quotient finite, Proc. Cambridge Philos. Soc. 69 (1971), 373-391. MR 0274575 (43 \#338)

[Wil04a] John S. Wilson, Further groups that do not have uniformly exponential growth, J. Algebra 279 (2004), no. 1, 292-301. MR 2078400 (2005e:20066)

[Wil04b] ㄴ On exponential growth and uniformly exponential growth for groups, Invent. Math. 155 (2004), no. 2, 287-303. MR 2031429

[Wil05] 느는 On the growth of residually soluble groups, J. London Math. Soc. (2) 71 (2005), no. 1, 121-132. MR 2108251 (2006a:20069)

[Woe00] Wolfgang Woess, Random walks on infinite graphs and groups, Cambridge Tracts in Mathematics, vol. 138, Cambridge University Press, Cambridge, 2000. MR 1743100 (2001k:60006)

[Wol68] Joseph A. Wolf, Growth of finitely generated solvable groups and curvature of Riemanniann manifolds, J. Differential Geometry 2 (1968), 421-446. MR 0248688 (40 \#1939)

[Yau75] Shing Tung Yau, Harmonic functions on complete Riemannian manifolds, Comm. Pure Appl. Math. 28 (1975), 201-228. MR 0431040 (55 \#4042)

[Zel90] E. I. Zel'manov, Solution of the restricted Burnside problem for groups of odd exponent, Izv. Akad. Nauk SSSR Ser. Mat. 54 (1990), no. 1, 42-59, 221. MR 1044047 (91i:20037)

[Zel91a] _ Solution of the restricted Burnside problem for 2-groups, Mat. Sb. 182 (1991), no. 4, 568-592. MR 1119009 (93a:20063)

[Zel91b] Efim I. Zelmanov, On the restricted Burnside problem, Proceedings of the International Congress of Mathematicians, Vol. I, II (Kyoto, 1990) (Tokyo), Math. Soc. Japan, 1991, pp. 395-402. MR 1159227 (93d:20076)

[Zel05] Efim Zelmanov, Infinite algebras and pro-p groups, Infinite groups: geometric, combinatorial and dynamical aspects, Progr. Math., vol. 248, Birkhäuser, Basel, 2005, pp. 403-413. MR 2195460 (2006k:20053)

[Zel07] S Some open problems in the theory of infinite dimensional algebras, J. Korean Math. Soc. 44 (2007), no. 5, 1185-1195. MR 2348741 (2008g:16053)

Department of Mathematics; Mailstop 3368; Texas A\&M University; College Station, TX 77843-3368, USA

E-mail address: grigorch@math.tamu.edu 